\title{
Initial Stages of Spontaneous Binding of Folate-based Vectors to Folate Receptor- $\alpha$ Observed by Unbiased Molecular Dynamics
}

Ethan N. Schaber, Nikoleta Ivanova, ${ }^{1}$ Stoyan Iliev, Jasmina Petrova, Gergana Gocheva, Galia Madjarova, Anela Ivanova*

Laboratory of Quantum and Computational Chemistry, Faculty of Chemistry and Pharmacy, Sofia University “St. Kliment Ohridski”, 1 James Bourchier blvd., 1164 Sofia, Bulgaria

*Corresponding author: e-mail: aivanova@chem.uni-sofia.bg; Tel.: ++35928161520; FAX ++35929625438

\section{Supporting Information}

${ }^{1}$ Current address: Department of Physical Chemistry, University of Chemical Technology and Metallurgy, Sofia, Bulgaria 


\section{Model systems and computational protocol}

Membrane/receptor model system:

370 lipid molecules of 35 different types are asymmetrically distributed between the two leaflets of the lipid bilayer. The negatively charged lipids are concentrated exclusively in the inner leaflet. The initial average area per lipid in each leaflet is $0.55 \mathrm{~nm}^{2}$ for the outer leaflet and $0.62 \mathrm{~nm}^{2}$ for the inner leaflet and the proportion of hydrophobic lipid tails is taken to be $61 \mathrm{~mol} \%$ fully saturated, $28 \mathrm{~mol} \%$ monounsaturated, and $11 \mathrm{~mol} \%$ polyunsaturated (PUFA). ${ }^{\mathrm{S} 1 \mathrm{S2}}$ The crystal structure of FR $\alpha$ as determined by Chen et al. ${ }^{S 3}$ (PDB ID: 4LRH) is used to model the receptor, encompassing 204 amino acid residues. The three glycosylated asparagine residues (47, 139, 179), as identified in the $X$-ray structure, are also preserved in the model. The protein has a total charge of +4 due to the incorporated charged amino acids (13 glutamate, 7 aspartate, 12 lysine, and 11 arginine residues; the $\mathrm{N}$-terminal glutamine also bears a positive charge). The total charge of the GPI anchor (see Ref. S1 for its chemical structure) is -1 due to two deprotonated phosphate residues and a single protonated amino group in glucosamine of the glycan core. The FR $\alpha$-GPI complex is anchored into the outer leaflet of the lipid bilayer.

According to the information available in the literature, ${ }^{\mathrm{S}} \mathrm{FR} \alpha$ is located predominantly in lipid rafts and experimental data show that from $60 \%$ to $90 \%$ of the receptors are anchored there as single molecules. In addition, it is known that five of the six ligands form a 1:1 ligand:receptor complex with affinity constant in the nanomolar range (see the main manuscript). This motivated the choice of the contents of the simulation models in the study.

MD simulations protocol:

First, an energy minimization is performed with the whole system restrained, except for the ligand, using the method of steepest descent with a gradient of $500 \mathrm{~kJ} \cdot \mathrm{mol}^{-1} \cdot \mathrm{nm}^{-1}$. This is followed by a fully unrestrained production run with initial velocities assigned randomly from a MaxwellBoltzmann distribution at $310 \mathrm{~K}$. Leap-frog with a time step of $2 \mathrm{fs}$ is used to integrate the equations of motion. Electrostatic interactions are described by $\mathrm{PME}^{\mathrm{S}}$ and van der Waals ones by a Lennard-Jones potential, both with a cut-off of $1.2 \mathrm{~nm}$ and a switching function effective at $1.0 \mathrm{~nm}$. The v-rescale thermostat ${ }^{56}$ with a coupling constant of $0.1 \mathrm{ps}$ is used to maintain the constant temperature and the Berendsen barostat ${ }^{S 7}$ with a 1 ps contact time and $4.5 \times 10^{-5}$ bar $^{-1}$ compressibility is employed to maintain the target pressure along $z$ and surface tension. The LINCS $^{58}$ algorithm is applied to fix the hydrogen-containing bond lengths for lipids, proteins and saccharides while SETTLE ${ }^{S 9}$ is used to constrain those of the water molecules.

The equilibration of the systems is monitored by estimating standard properties - the total energy, temperature, periodic box length in the z-direction, density, pressure in the zdirection, and surface tension of the system, as well as the temperature of the individual components ( $\mathrm{Na}^{+}$ions, $\mathrm{Cl}^{-}$ions, water molecules, lipid bilayer, receptor, and ligand). The RMSD of the atomic coordinates of the ligand, the protein, and the GPI (see below) is calculated and tracked, too. The energy-minimized structure is used as reference.

The minimum distance and number of contacts within a distance of $0.6 \mathrm{~nm}$ between FR $\alpha$ and the ligand are computed to assess the binding of the vector molecules. 
Table S1: Description of the contents of the model systems; $N$ denotes the respective number of molecules/ions; $\mathrm{N}_{\text {total }}$ is the total number of atoms in the model; all models contain also $1 \mathrm{GPI}$ anchored receptor molecule, 1 ligand and 370 lipids in the bilayer

\begin{tabular}{|c|c|c|c|c|c|c|}
\hline Ligand & Trajectory & $\mathbf{N}_{\text {water }}$ & $\mathrm{N}_{\mathrm{Na}+}$ & $\mathbf{N}_{\mathrm{Cl}-}$ & $\mathbf{N}_{\text {total }}$ & $\begin{array}{c}\text { Initial box size } x \times y \times z \\
n m\end{array}$ \\
\hline \multirow[t]{4}{*}{ FA } & 1 & 45601 & 244 & 190 & 184868 & $10.7 \times 10.7 \times 18.2$ \\
\hline & 2 & 45575 & 244 & 190 & 184790 & $10.7 \times 10.7 \times 18.2$ \\
\hline & 3 & 45587 & 244 & 190 & 184826 & $10.6 \times 10.6 \times 18.7$ \\
\hline & 4 & 45575 & 244 & 190 & 184790 & $10.7 \times 10.7 \times 18.3$ \\
\hline \multirow[t]{4}{*}{ MTX } & 1 & 45601 & 244 & 190 & 184872 & $10.7 \times 10.7 \times 18.2$ \\
\hline & 2 & 45575 & 244 & 190 & 184794 & $10.7 \times 10.7 \times 18.2$ \\
\hline & 3 & 45587 & 244 & 190 & 184830 & $10.6 \times 10.6 \times 18.7$ \\
\hline & 4 & 45575 & 244 & 190 & 184794 & $10.7 \times 10.7 \times 18.3$ \\
\hline \multirow[t]{4}{*}{ RTX } & 1 & 45601 & 244 & 190 & 184871 & $10.7 \times 10.7 \times 18.2$ \\
\hline & 2 & 45575 & 244 & 190 & 184793 & $10.7 \times 10.7 \times 18.2$ \\
\hline & 3 & 45587 & 244 & 190 & 184829 & $10.6 \times 10.6 \times 18.7$ \\
\hline & 4 & 45575 & 244 & 190 & 184793 & $10.7 \times 10.7 \times 18.3$ \\
\hline \multirow[t]{4}{*}{ PTX } & 1 & 45601 & 244 & 190 & 184869 & $10.7 \times 10.7 \times 18.2$ \\
\hline & 2 & 45575 & 244 & 190 & 184791 & $10.7 \times 10.7 \times 18.2$ \\
\hline & 3 & 45587 & 244 & 190 & 184827 & $10.6 \times 10.6 \times 18.7$ \\
\hline & 4 & 45575 & 244 & 190 & 184791 & $10.7 \times 10.7 \times 18.3$ \\
\hline \multirow[t]{4}{*}{ MTHF } & 1 & 45601 & 244 & 190 & 184875 & $10.7 \times 10.7 \times 18.2$ \\
\hline & 2 & 45575 & 244 & 190 & 184797 & $10.7 \times 10.7 \times 18.2$ \\
\hline & 3 & 45587 & 244 & 190 & 184833 & $10.6 \times 10.6 \times 18.7$ \\
\hline & 4 & 45575 & 244 & 190 & 184797 & $10.7 \times 10.7 \times 18.3$ \\
\hline \multirow[t]{4}{*}{ PON } & $\overline{11}$ & 445601 & 243 & 191 & 184872 & $10.7 \times 10.7 \times 18.2$ \\
\hline & 2 & 45575 & 243 & 191 & 184794 & $10.7 \times 10.7 \times 18.2$ \\
\hline & 3 & 45587 & 243 & 191 & 184830 & $10.6 \times 10.6 \times 18.7$ \\
\hline & 4 & 45575 & 243 & 191 & 184794 & $10.7 \times 10.7 \times 18.3$ \\
\hline
\end{tabular}




\section{Ligand binding times and duration}

Table S2: Total production trajectory time $\left(t_{\text {traj }}\right)$, time of first binding $\left(t_{\text {bind }}\right)$, and segment of the trajectory in bound state of each ligand to the receptor in all trajectories

\begin{tabular}{|c|c|c|c|c|c|}
\hline Ligand & Trajectory & $t_{\text {traj, }} \mathbf{n s}$ & $t_{\text {bind, }}$ ns & Bound time, ns & Bound period, ns \\
\hline \multirow[t]{4}{*}{ FA } & 1 & 300 & 175 & $\min .125$ & $175-300$ \\
\hline & 2 & 400 & 280 & $\min .120$ & $280-400$ \\
\hline & 3 & 300 & 50 & 68 & $50-118$ \\
\hline & 4 & 250 & 20 & 138 & $20-60 / 80-138 / 200-240$ \\
\hline \multirow[t]{4}{*}{ MTX } & 1 & 200 & --- & 0 & --- \\
\hline & 2 & 200 & 100 & 58 & $100-158$ \\
\hline & 3 & 200 & --- & 0 & --- \\
\hline & 4 & 250 & 18 & 112 & $18-80 / 120-170$ \\
\hline \multirow[t]{4}{*}{ RTX } & 1 & 300 & 150 & $\min .100$ & $150-250$ \\
\hline & 2 & 200 & 9 & $\min .148$ & $9-48 / 65-127 / 153-200$ \\
\hline & 3 & 200 & 18 & $\min .182$ & $18-200$ \\
\hline & 4 & 250 & 42 & $\min .208$ & $42-250$ \\
\hline \multirow[t]{4}{*}{ PTX } & 1 & 250 & 0 & 248 & $0-248$ \\
\hline & 2 & 250 & 160 & $\min .90$ & $160-250$ \\
\hline & 3 & 200 & 0 & 140 & $0-140$ \\
\hline & 4 & 250 & 10 & $\min .240$ & $10-250$ \\
\hline \multirow[t]{4}{*}{ MTHF } & 1 & 200 & 20 & $\min .180$ & $20-200$ \\
\hline & 2 & 250 & 0 & 130 & 0-70/135-195 \\
\hline & 3 & 200 & 0 & 130 & $0-130$ \\
\hline & 4 & 250 & 37 & 203 & $37-240$ \\
\hline \multirow[t]{4}{*}{ PON } & 1 & 250 & 125 & 90 & $125-215$ \\
\hline & 2 & 200 & --- & 0 & --- \\
\hline & 3 & 200 & --- & 0 & --- \\
\hline & 4 & 250 & --- & 0 & --- \\
\hline
\end{tabular}


Minimum distance and number of contacts between the ligands and FR $\alpha$
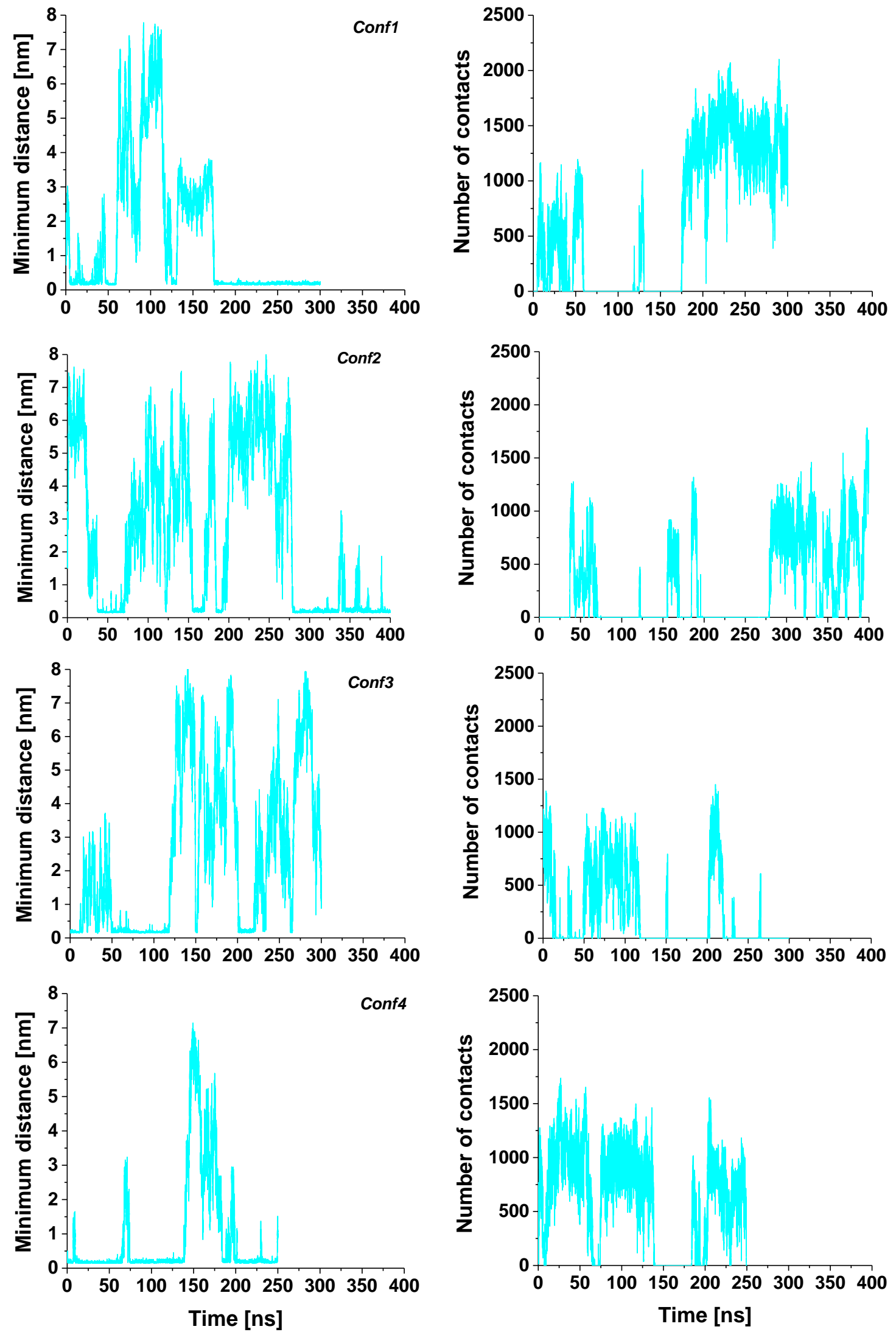

Figure S1: Evolution of the (left) minimum distance and (right) number of contacts between atom pairs from FA and FR $\alpha$ in the four MD trajectories 

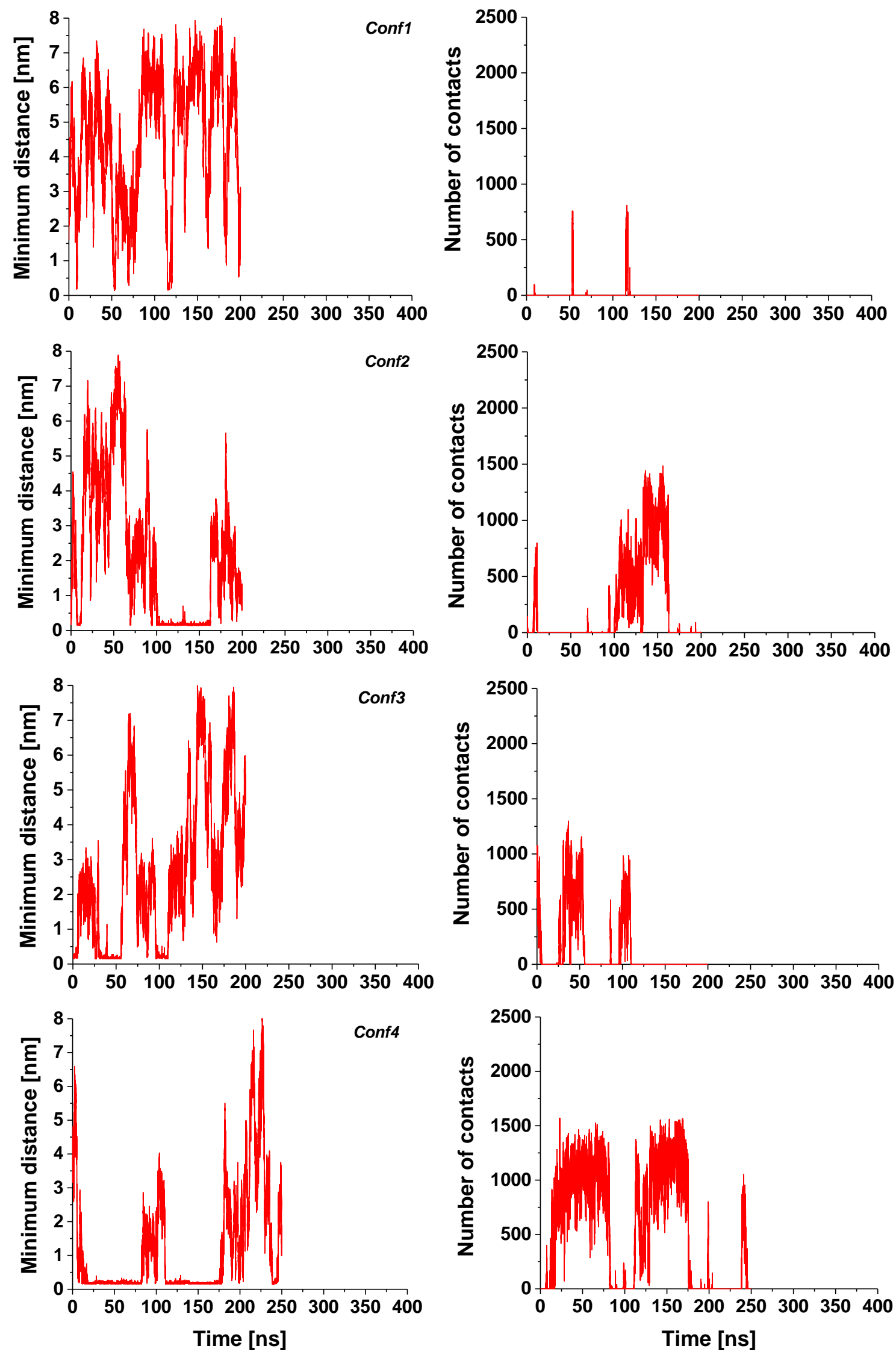

Figure S2: Evolution of the (left) minimum distance and (right) number of contacts between atom pairs from MTX and FR $\alpha$ in the four MD trajectories 

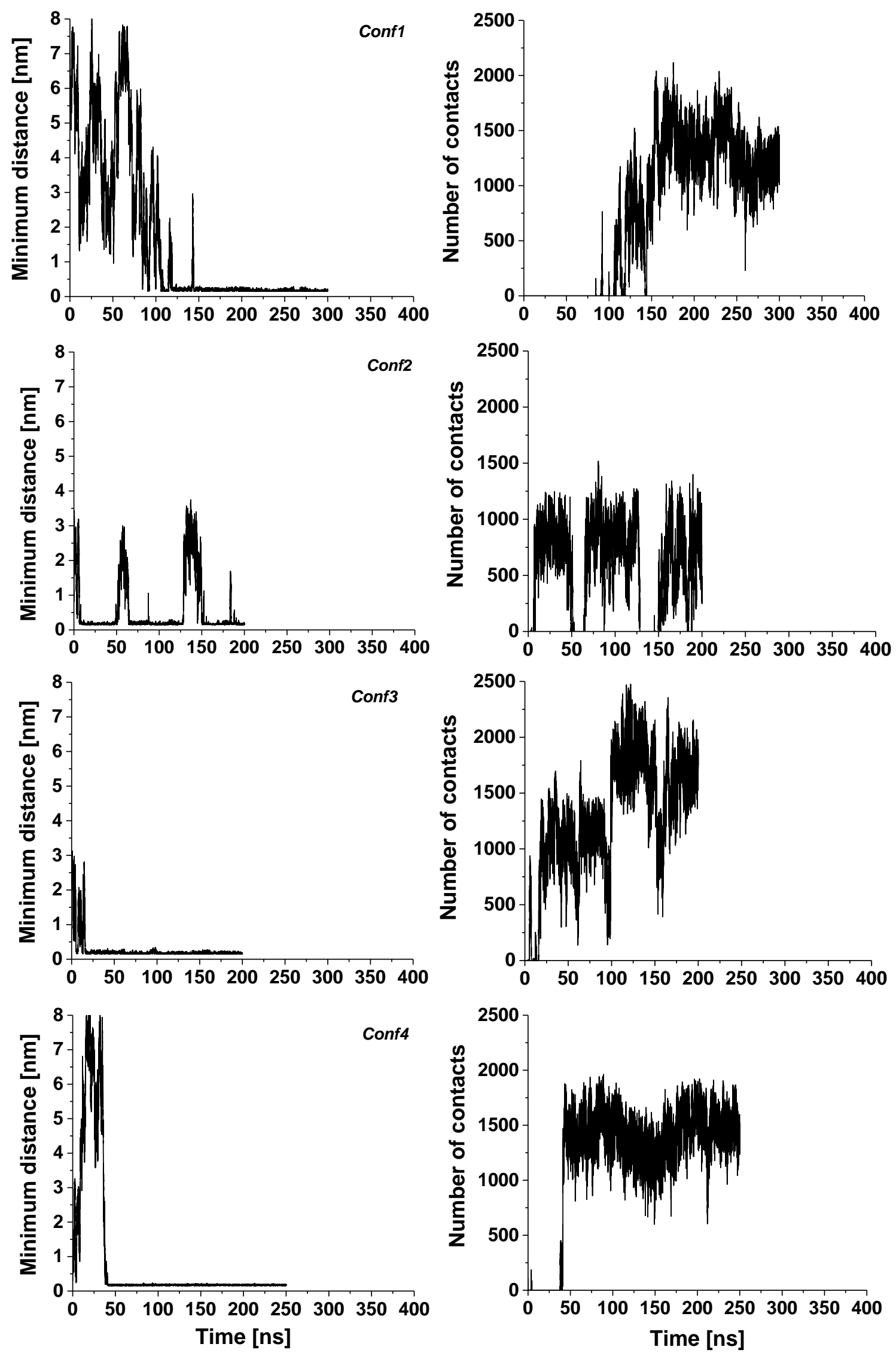

Figure S3: Evolution of the (left) minimum distance and (right) number of contacts between atom pairs from RTX and FR $\alpha$ in the four MD trajectories 

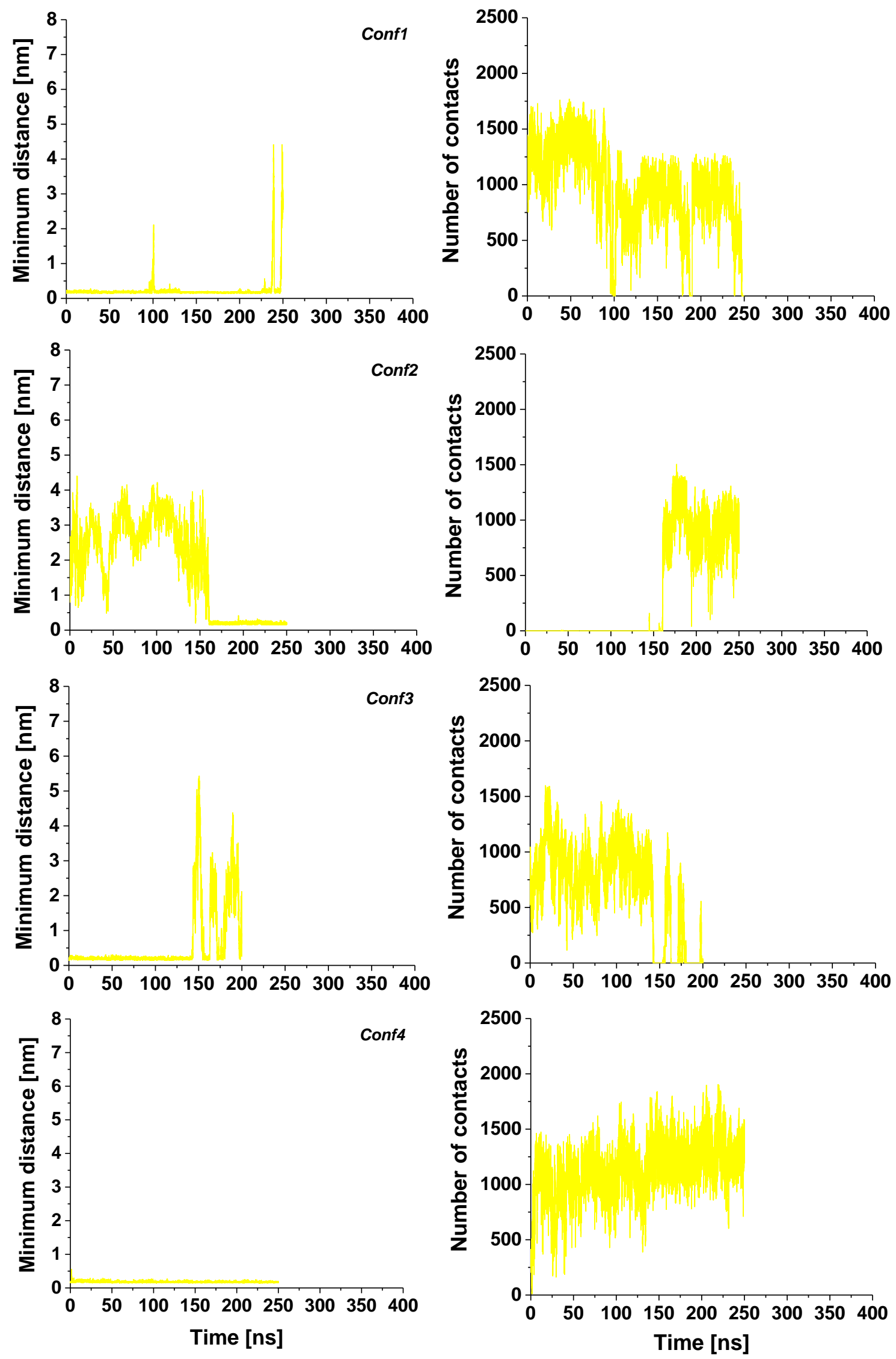

Figure S4: Evolution of the (left) minimum distance and (right) number of contacts between atom pairs from PTX and FR $\alpha$ in the four MD trajectories 

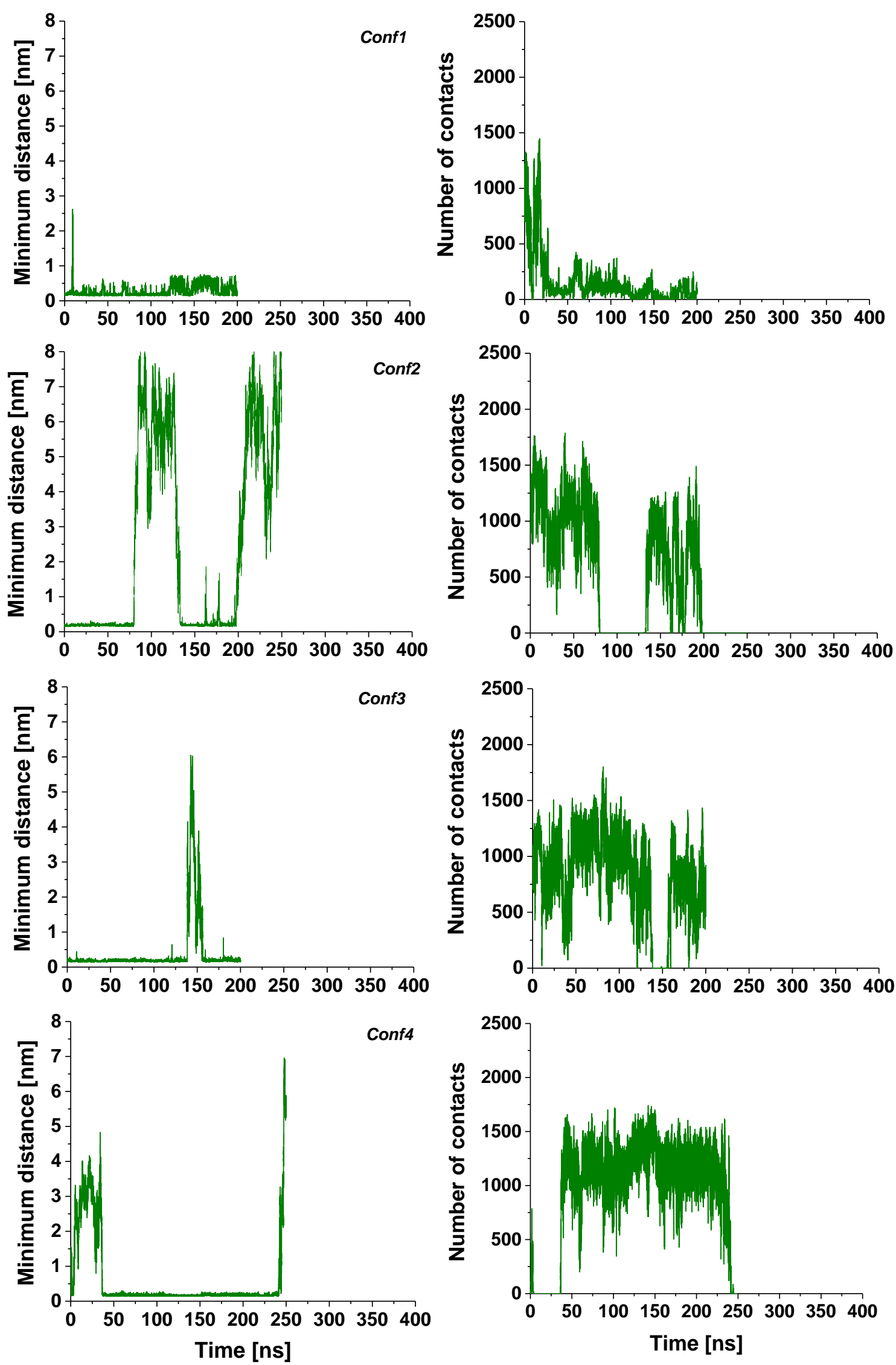

Figure S5: Evolution of the (left) minimum distance and (right) number of contacts between atom pairs from MTHF and FR $\alpha$ in the four MD trajectories 

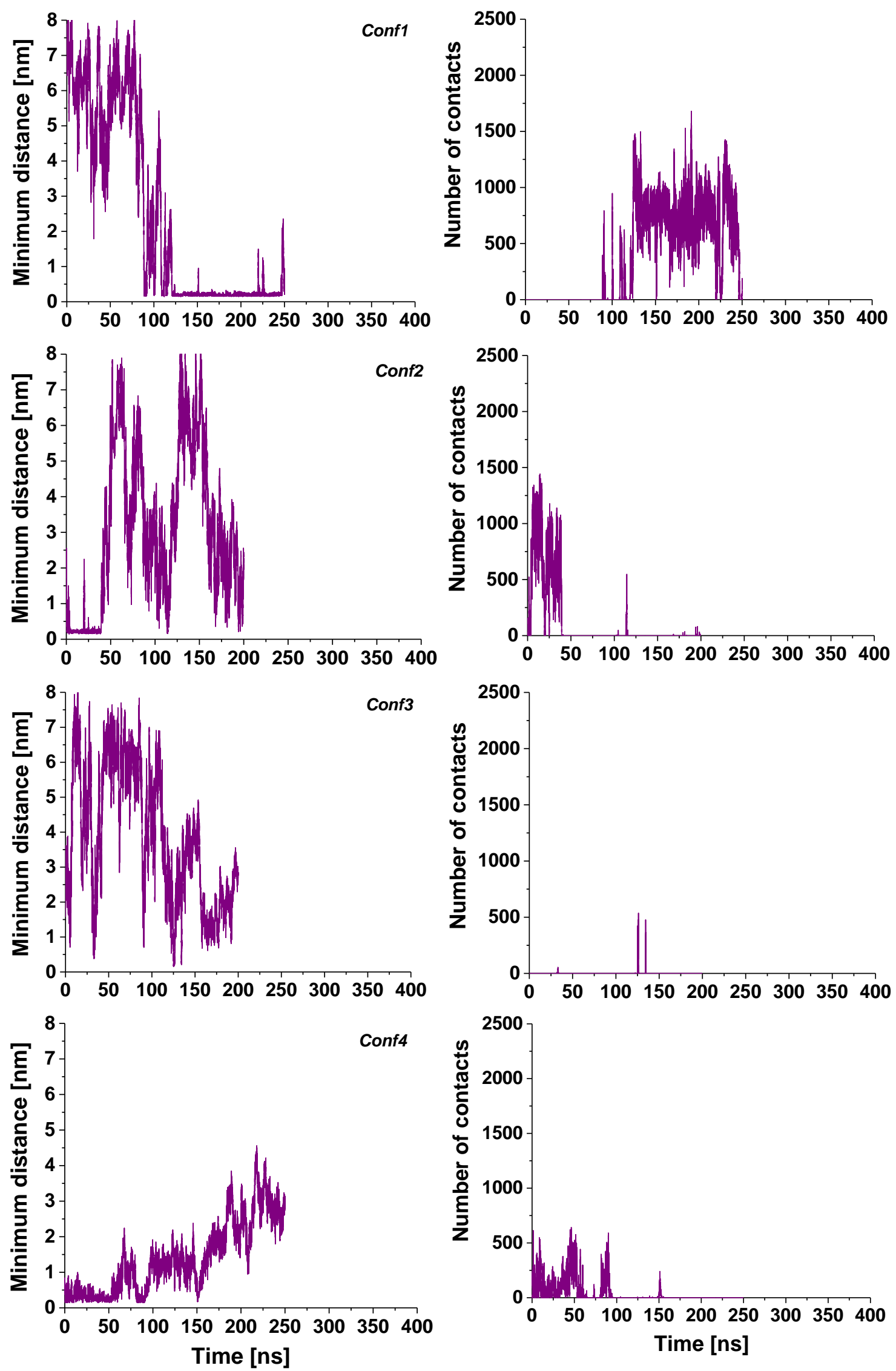

Figure S6: Evolution of the (left) minimum distance and (right) number of contacts between atom pairs from PON and FRa in the four MD trajectories 


\section{Experimental affinity of FA and its derivatives for FR $\alpha$}

The dissociation constants of PTX and MTX are measured by Wibowo et al. ${ }^{\mathrm{S} 10}$ by isothermal titration calorimetry experiment to be $K_{d}=4.5 \mathrm{nM}$ and $K_{d}=65 \mathrm{nM}$, respectively. Mauritz et al. ${ }^{\text {S11 }}$ outline the affinity of MTX, RTX and PTX relative to FA (whose affinity was set to 1) for FR $\alpha$ expressed in L1210-RFC cells, a leukemic murine cell line, with a competitive binding assay at $\mathrm{pH}$ 7.4. While MTX and RTX have lower relative affinities (0.009 and 0.68$)$, the relative affinity of PTX to FR $\alpha$ is reported at 1.48. The relative affinity is defined as the inverse molar ratio of molecules needed to displace $50 \%$ of the bound folate. Similar values are found by Theti and Jackman $^{\mathrm{S12}}$ who study the affinity of antifolates for the FR $\alpha$ in murine L1210-FBP and in human A431-FBP cells transfected with the receptor at $\mathrm{pH}<8.0$. In this study, MTX displays less than 0.01 relative affinity, RTX shows 0.61 in mice and 0.24 in human, and PTX displays 1.2 affinity in mice and 1.8 in human relative to FA. The affinity of RTX in human KB cells is 0.31 .

Measurement of the dissociation constants of FA, MTHF, and MTX upon binding to folate receptors expressed on the surface of human KB cells at $\mathrm{pH} 7.5$ yields values of $0.35 \mathrm{nM}, 1.0 \mathrm{nM}$, and $113.8 \mathrm{nM}$, respectively. ${ }^{\mathrm{s} 13}$ 


\section{Location of the ligands in the bound periods}

\section{Folate}

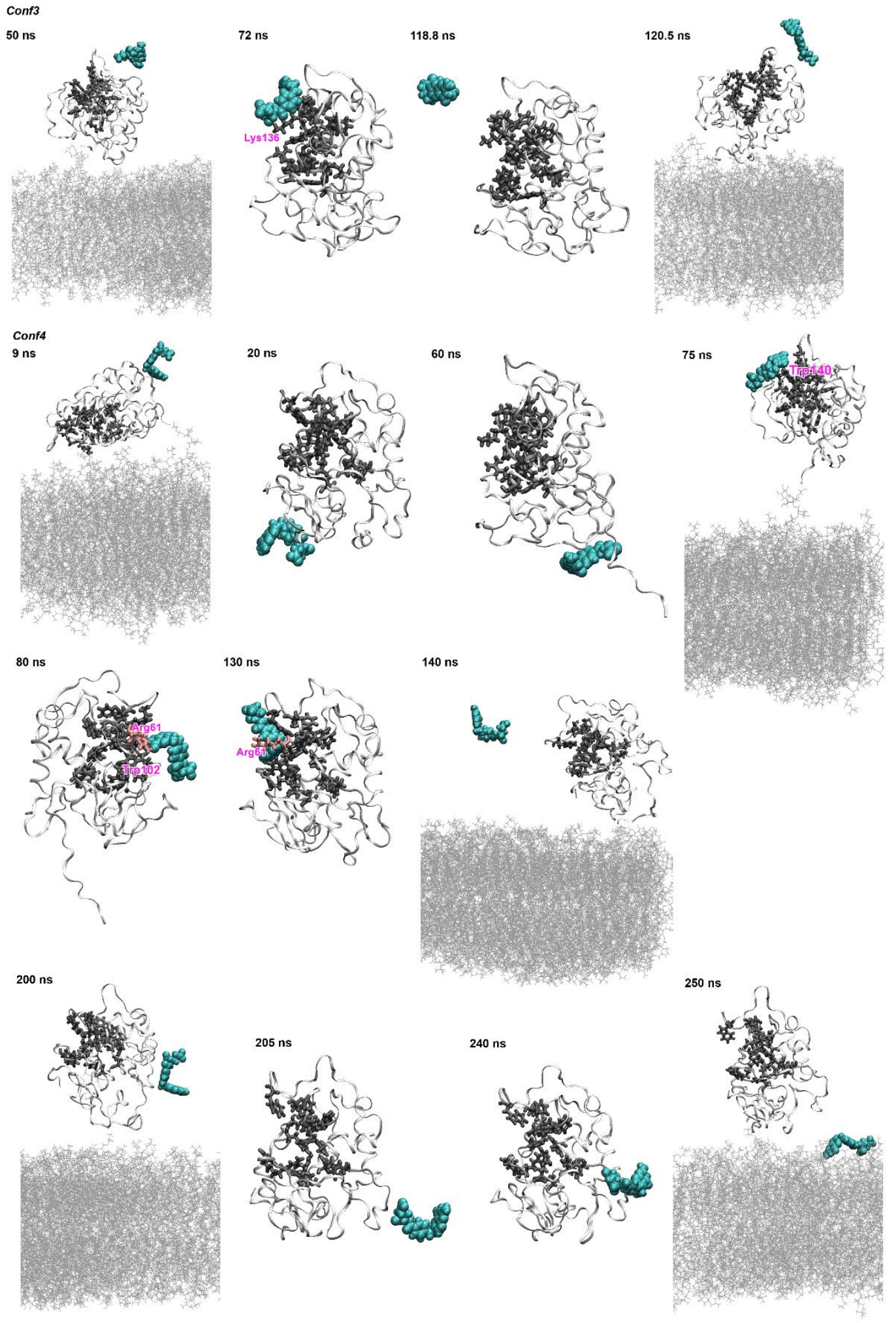

Figure S7: Representative snapshots from the Conf3 and Conf4 trajectories of folate illustrating its binding to FR $\alpha$; the membrane lipids are in silver lines, the protein - in white ribbons, its ligandbinding pocket - in grey licorice, Arg61 - in pink licorice, and FA - in cyan spheres; water and inorganic ions are omitted for clarity; key interacting amino acids are labeled in magenta 
Before binding in Conf1, FA diffuses across the entire model system. Just prior to its attachment to $F R \alpha$, it interacts with lipids in the membrane, far from the receptor. Then, over a very short period ( $\sim 500 \mathrm{ps)}$ ) the ligand moves straight to the binding pocket and attaches there (Figure 4, top). First, pterin tries to enter the cavity but is unsuccessful, then the glutamate is attracted shortly by Lys136. After that pterin is $\pi$-stacked by Trp140 and finally the aminobenzoate is positioned into the pocket entrance, coupled to Trp140. At the same time, Lys136 interacts with the glutamate. FA remains bound with this part of the molecule until the end of the trajectory. In the bound period, it readjusts, sometimes occupying very tightly the pocket entrance. The aminobenzoate $\pi$-stacks Trp140 constantly, sometimes accompanied by Trp102. The latter results in intercalation of FA. Both carboxylate groups of the glutamate interact electrostatically with Lys136.

In Conf2, just before the binding FA 'swims' in the solution around FR $\alpha$, at the side opposite the binding site (Figure 4, middle). Within 1 ns it approaches the pocket with pterin, then for ca. 500 ps faces it with glutamate, then again with pterin, which remains bound. Trp140 is the first interaction partner. Pterin stays $\pi$-stacked to it for some time and this happens repeatedly during the bound period. From 337 ns to 358.5 ns the ligand detaches from the protein. Then, it interacts shortly with amino acids away from the pocket and later on reassumes the same pterin-Trp140 $\pi$-stacked orientation and remains fluctuating at the pocket entrance. Around $387.5 \mathrm{~ns}$ the ligand drags Trp140 quite far apart from the rest of the protein, which opens the pocket. This seems to be unfavorable, since at 390 ns FA detaches again shortly from FR $\alpha$ but soon comes back to Trp140. At 395 ns folate tries to enter the cavity with glutamate but is expelled. Overall, the dominant and persistent interaction in this trajectory is the $\pi$-stacking pterin-Trp140. The ligand is very mobile at the pocket entrance, in general less inserted than in Conf1. It is noteworthy that in the entire bound period FR $\alpha$ is bent toward the membrane and the binding pocket faces the lipid bilayer.

In Conf3, FA comes from above FR $\alpha$, spends a short time at the loop on top of the binding site and then goes to the pocket and remains there for the entire bound period (Figure S7, top). In this case, the pocket points away from the membrane in the entire interaction segment. At 71.5 ns pterin shortly couples to Trp140, then FA wanders non-specifically in front of the pocket and after 90 ns remains bound via glutamate attracted by Lys136 or Arg106 and then by both of them in a clamp-like manner. This is stabilized by an additional interaction of pterin with Trp140 (until 106 ns) or of aminobenzoate with Trp102 (106-110 ns). At 113 ns glutamate is released from the clamp almost instantaneously and FA starts fluctuating in front of the pocket. At $121 \mathrm{~ns}$ it dissociates into the solution above FRa. This behavior indicates that such electrostatically dominated attraction of glutamate is not sufficient to hold the ligand to the pocket for a long time.

In Conf4, FA spends the first bound period (20-60 ns) away from the pocket at the base of the protein (Figure S7, second row). It is noteworthy that in this segment of the trajectory the C- 
terminus of FR $\alpha$ is largely unfolded, leaving space for interaction between FA and the GPI sugars. In this trajectory segment, the pocket points toward the membrane and FA is already close to the protein base from the beginning of the MD production stage. After 10ns FR $\alpha$ starts standing up from the membrane and FA also moves a bit upward the protein surface. After $15 \mathrm{~ns}$ the ligand moves forth and back between the tip of the protein base and the vicinity of the GPI until 69.9 ns. Then, FA shortly approaches the GPI and dissociates. After a quick rearrangement, FA attaches to the pocket and stays close to it in the second bound part (80-138 ns) (Figure S7, third row). There, pterin is attracted initially by Trp102, then by Trp140 to which it $\pi$-stacks. After that it $\pi$ stacks shortly with Trp102 and then interacts mostly with Arg61. The latter is accomplished by $\pi$ stacking of pterin or aminobenzoate to guanidinium or by electrostatic attraction of glutamate to it. FA fluctuates very dynamically at the pocket entrance during the entire bound period. It does not couple tightly to any pocket residue. At $138.5 \mathrm{~ns}$ it dissociates and dissolves. In the third bound trajectory piece (200-240 ns) FA comes from the 'back' of FR $\alpha$, which is upright with the pocket oriented sideways (Figure S7, bottom). The ligand is mostly non-specifically adsorbed on the surface of FR $\alpha$, opposite the binding site. It is very mobile there, sometimes reaching also the GPI. The behavior is similar to that in the first bound period. At $249.5 \mathrm{~ns}$ FA dissociates and moves to the membrane. It does not readsorb until the end of the simulation. This very dynamic picture implies that in this trajectory the ligand did not encounter an appropriate arrangement of the binding site residues, which would allow it to remain firmly bound.

\section{Methotrexate}

In Conf2, shortly before 100 ns MTX interacts with the lipids from the outer leaflet of the bilayer. It then detaches from there and heads to the receptor. Even during the bound period of this trajectory, the glutamate of the ligand occasionally makes contacts with the membrane. As far as FR $\alpha$ is concerned, MTX is bound at the turn at the base of the protein facing the bilayer but far from the GPI anchor (Figure S9, top). The glutamate fragment interacts with the protein but the molecule changes its orientation relative to it. At $137 \mathrm{~ns}$ MTX spreads parallel to the receptor surface and remains like this until 158 ns. From 132.5 ns on mostly the aminobenzoate makes contact with FR $\alpha$ and only at the very end aminopterin (APT) comes into play. While bound, MTX remains at the same spot on the macromolecule surface. At 159 ns the ligand starts reducing the degree of contact with $\mathrm{FR} \alpha$, at $163 \mathrm{~ns}$ it completely separates from the protein after orienting its glutamate fragment toward it and at $164.3 \mathrm{~ns}$ MTX buries in the membrane and remains there. 

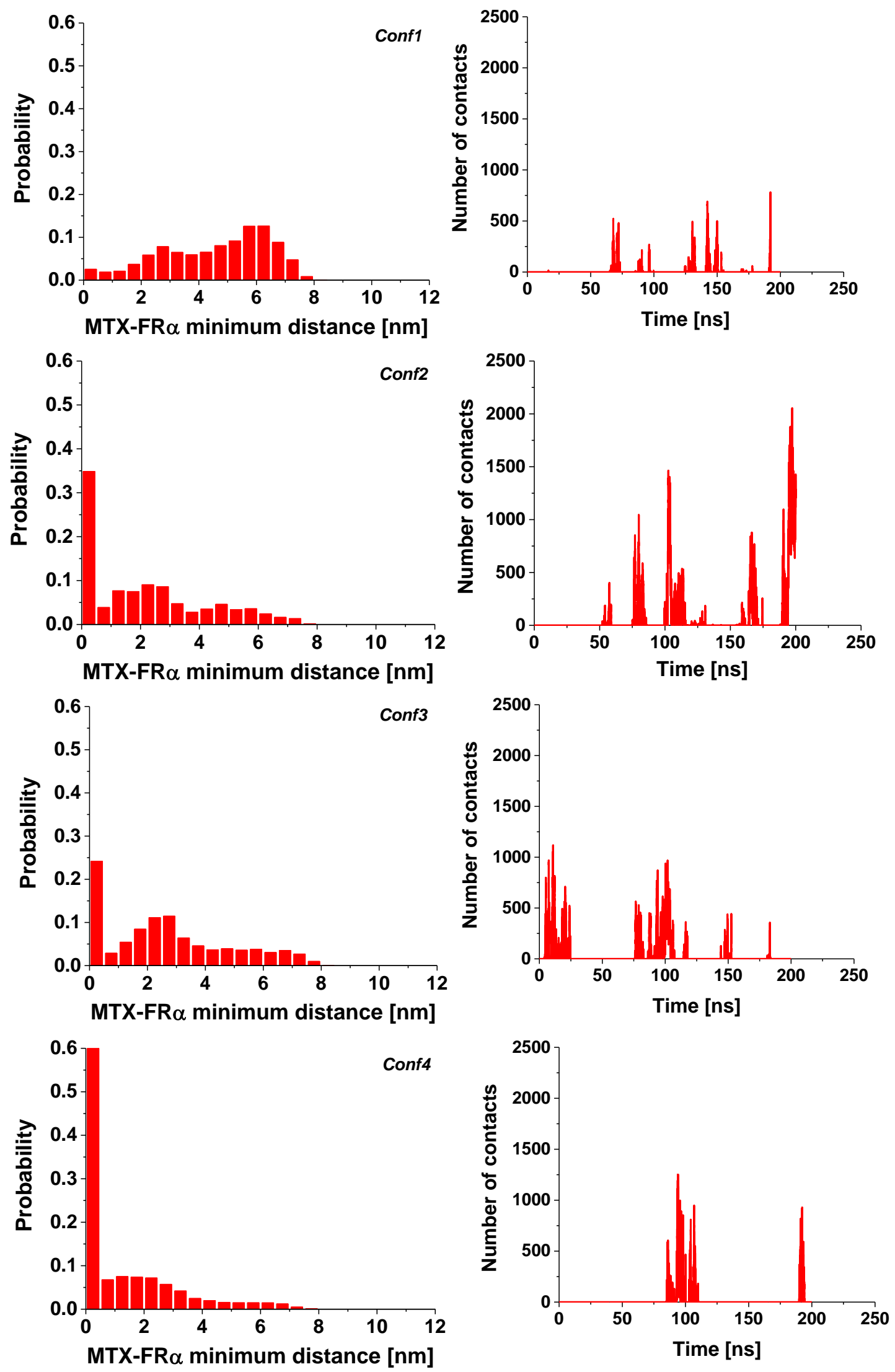

Figure S8: (left) Distribution of the minimum distance between MTX and FR $\alpha$ and (right) number of contacts between MTX and the membrane in the four MD trajectories 


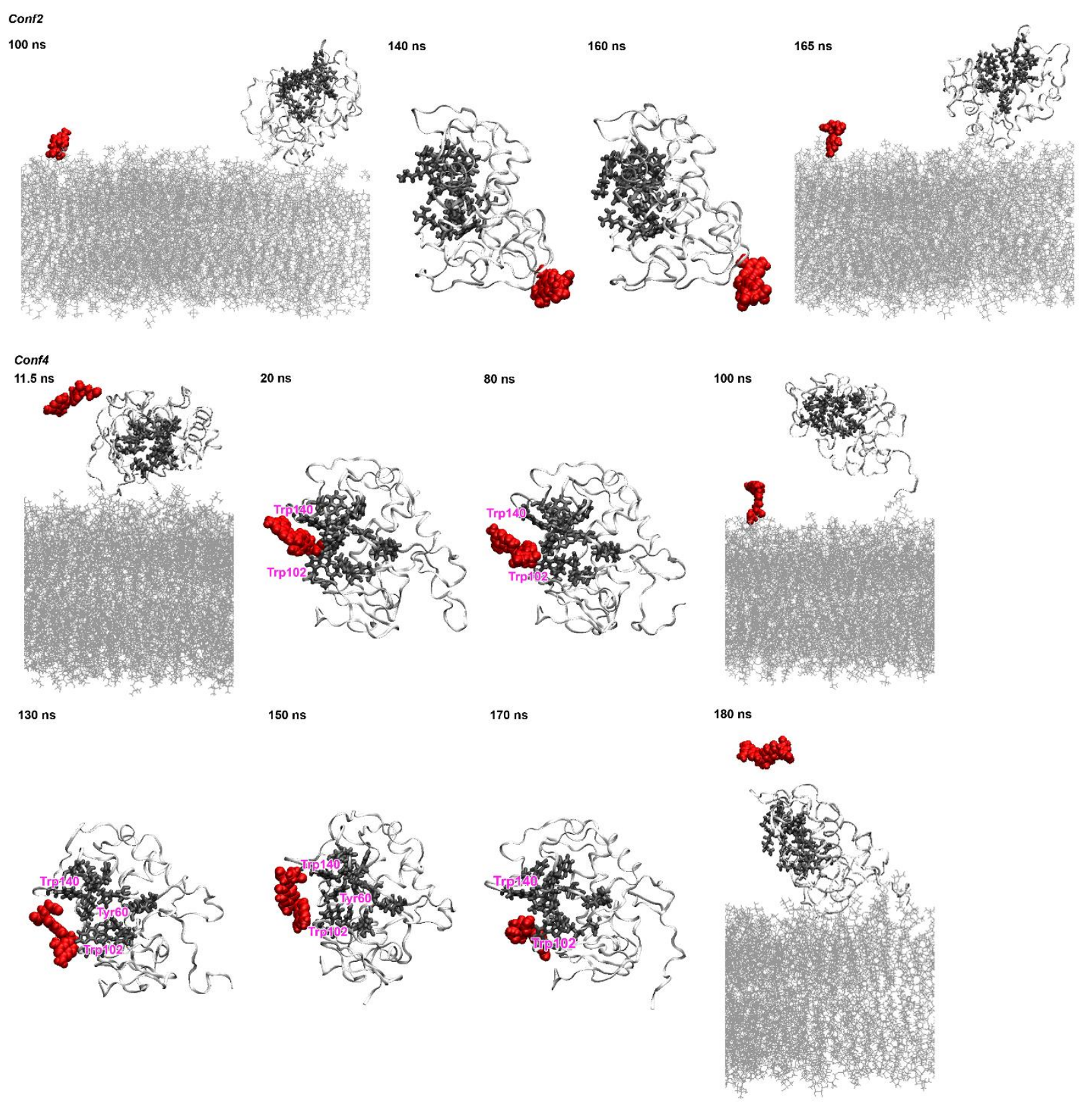

Figure S9: Representative snapshots from Conf2 and Conf4 trajectories of MTX illustrating its binding to FR $\alpha$; the membrane lipids are in silver lines, the protein - in white ribbons, its ligandbinding pocket - in grey licorice, and MTX - in red spheres; water and inorganic ions are omitted for clarity; key interacting amino acids are labeled in magenta

In the first bound period of Conf4, MTX approaches the protein from above. At 6.4 ns and at $7.1 \mathrm{~ns}$ it touches the top of the receptor but swiftly goes away. Then it moves closer and at 11.5 ns finally binds at the top (Figure S9, middle). After that the ligand scans the protein surface, probing various orientations and at $18 \mathrm{~ns}$ is already tightly coupled. At the beginning of this first bound period, MTX is bound next to the pocket, close to Trp140, its glutamate part interacting with this amino acid. At the same time aminopterin $\pi$-stacks with Trp102 and occasionally tries to enter the cavity but with no success. Part of the reason for the latter may be that Trp60 is 
located in the middle of the pocket entrance and blocks it. The ligand remains close to the pocket entrance during the entire first bound period (18-80 ns) but is very mobile. From 70 ns to 80 ns MTX is quite tightly bound with aminobenzoate to FR $\alpha$. At $81.7 \mathrm{~ns}$ the ligand detaches from the pocket, at $84 \mathrm{~ns}$ it dissociates from FR $\alpha$ and goes straight to the membrane. Before the second bound period (120-170 ns), MTX is in the membrane (Figure S9, snapshot at $100 \mathrm{~ns}$ ). At $111.5 \mathrm{~ns}$ it comes close to the pocket and binds. During this bound trajectory fragment, the molecule is located first 'at the back' of the protein, opposite to the pocket. It scans the surface there, then at $130 \mathrm{~ns}$ approaches very fast the binding pocket. From $135 \mathrm{~ns}$ on MTX binds in the same way as in the first bound segment of Conf4: glutamate interacts with Trp140 and aminopterin - with Trp102. The ligand remains like this, spanning the entire pocket entrance until $170 \mathrm{~ns}$. Then it detaches from Trp140, makes a 90-degree turn and couples solely to Trp102. At 175.5 ns MTX dissociates from the receptor and floats to the solution above it (Figure S9, bottom).

\section{Raltitrexed}

RTX binds to the active site of the receptor in Conf1 and in Conf3 and to another spot on the protein surface in Conf2 and Conf4.

In Conf1, already at 120 ns RTX is situated at the loop on top of the protein, close to the pocket (Figure 5). It goes away for a short time ( $\sim 10$ ps) and then comes back scanning the area around the pocket mostly with its glutamate part. At $125 \mathrm{~ns}$ the ligand tries entering the pocket with this fragment but unsuccessfully. This is repeated at $140 \mathrm{~ns}$. Finally, at $145 \mathrm{~ns}$ RTX manages to bind with MQ. At $150 \mathrm{~ns} \mathrm{MQ}$ is $\pi$-stacked by Trp140 and, at the same time, the glutamate of RTX forms hydrogen bonds with the backbone of this amino acid residue. Within $50 \mathrm{ps}$, MQ enters the pocket and reaches about its middle. Then, RTX adjusts its position inside the pocket until the end of the trajectory. Trp102 interacts all the time with MQ, supported also by His 135 and Lys 136. After 225 ns RTX retreats from the middle to the entrance of the pocket but remains bound.

At $5.5 \mathrm{~ns}$ of Conf3, the ligand approaches FR $\alpha$ from the membrane and stays close to the $\mathrm{N}$-terminus. At $8.5 \mathrm{~ns}$ it goes again to the membrane. It then surrounds the protein and at $16.5 \mathrm{~ns}$ approaches from the solution its surface with glutamate facing it. At $18.5 \mathrm{~ns}$ RTX binds at the pocket entrance both with this fragment and with MAT and remains there until 158 ns (Figure S10). It interacts predominantly and simultaneously with Trp140, Trp102 (MAT), and Lys136 (Glu). Occasionally, RTX goes closer to Trp140 only (in the period $37 \mathrm{~ns}$ to $60.8 \mathrm{~ns}$ ). From $60.8 \mathrm{~ns}$ to 63.5 ns, it rotates by $90^{\circ}$ and orients with MQ facing Trp140 and Glu being close to Trp102 (Figure S10). At $71 \mathrm{~ns}, \mathrm{RTX}$ transfers primarily near Trp102. At $78 \mathrm{~ns}$, it rotates back and intercalates the MAT part between Trp140 and Trp102. The ligand then spends time close to Trp102 until $95 \mathrm{~ns}$. It then moves forth and back between the two tryptophans and between the two types of rotated orientations. From $100 \mathrm{~ns}$ on, RTX is tightly bound at the pocket entrance in the initial alignment. The position of its glutamate is stabilized by a complementing interaction with Arg106. 

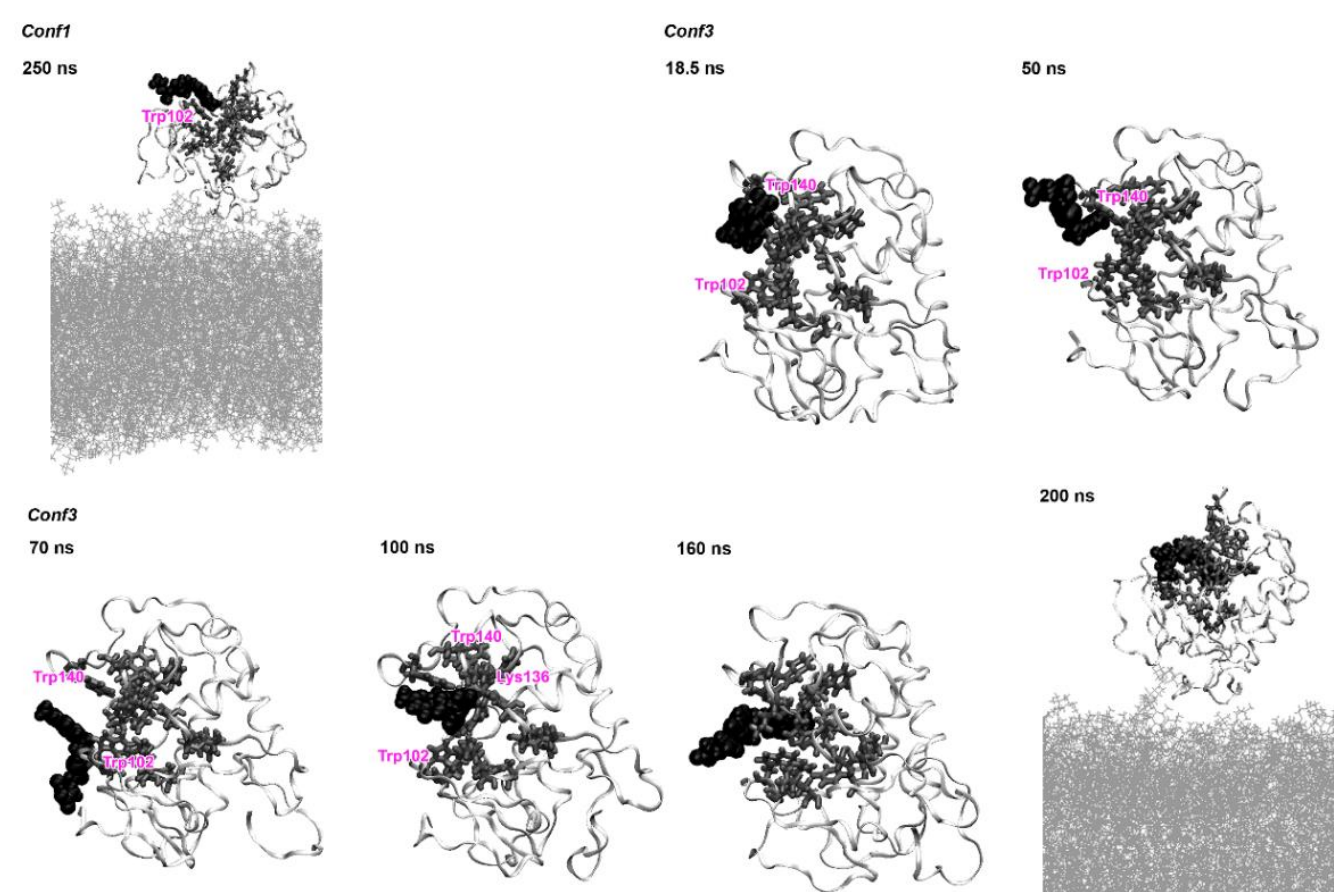

$160 \mathrm{~ns}$
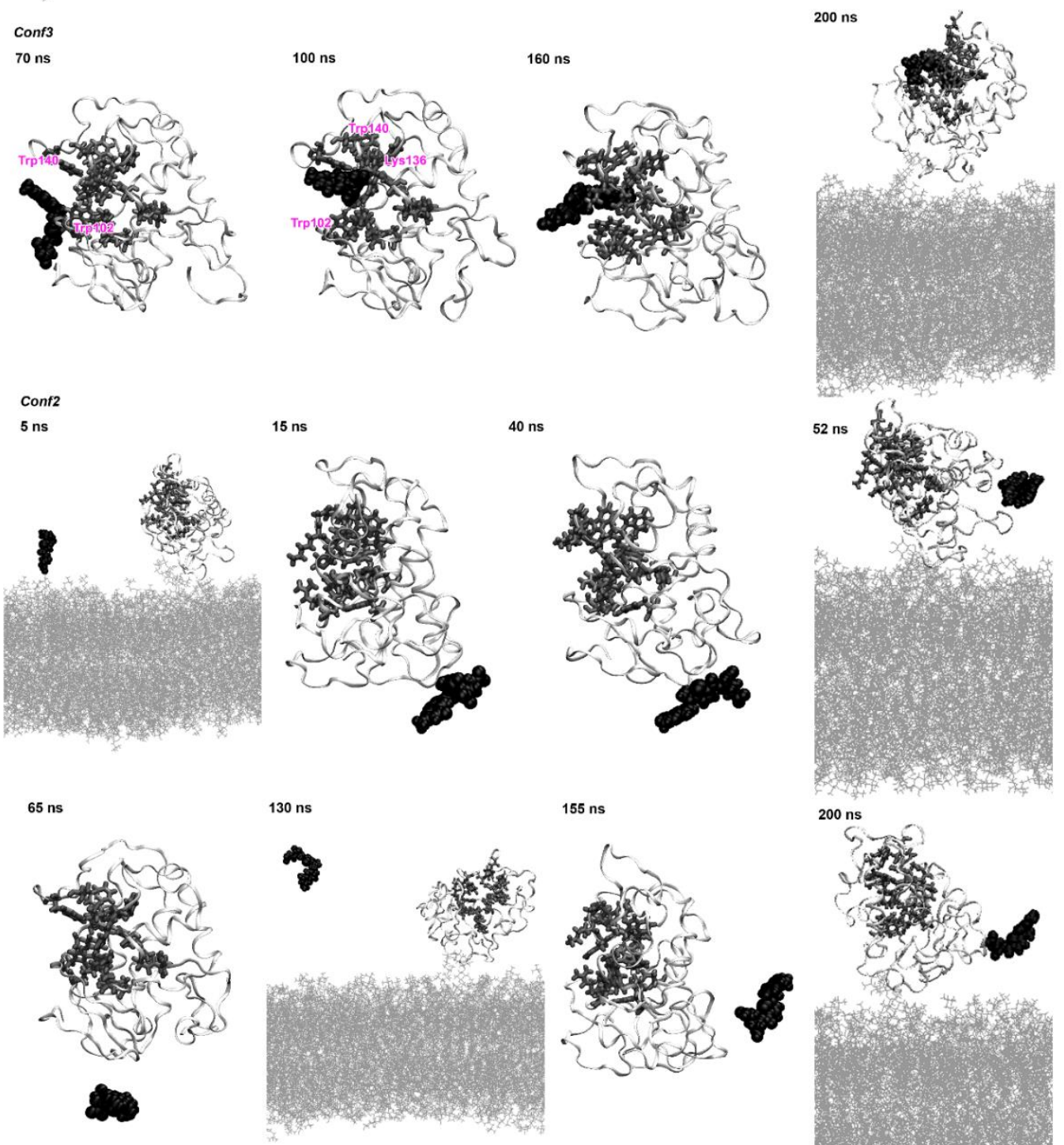

$155 \mathrm{~ns}$
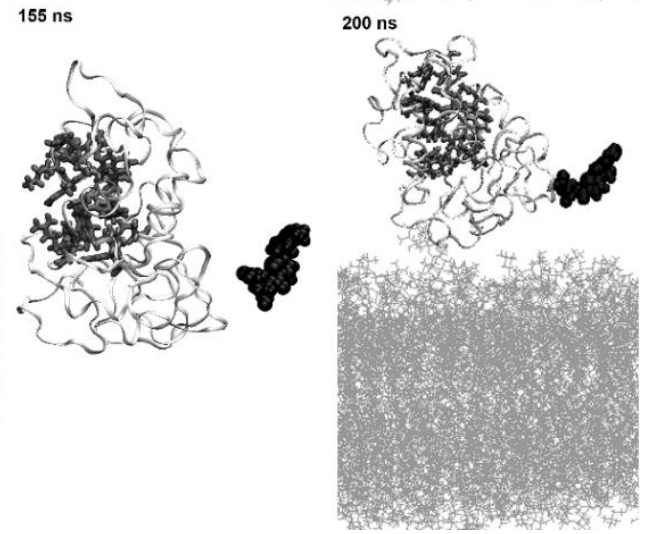

Figure S10: Representative snapshots from the trajectories of RTX illustrating its binding to FR $\alpha$; the membrane lipids are in silver lines, the protein - in white ribbons, its ligand-binding pocket in grey licorice, and RTX - in black spheres; water and inorganic ions are omitted for clarity; key interacting amino acids are labeled in magenta 


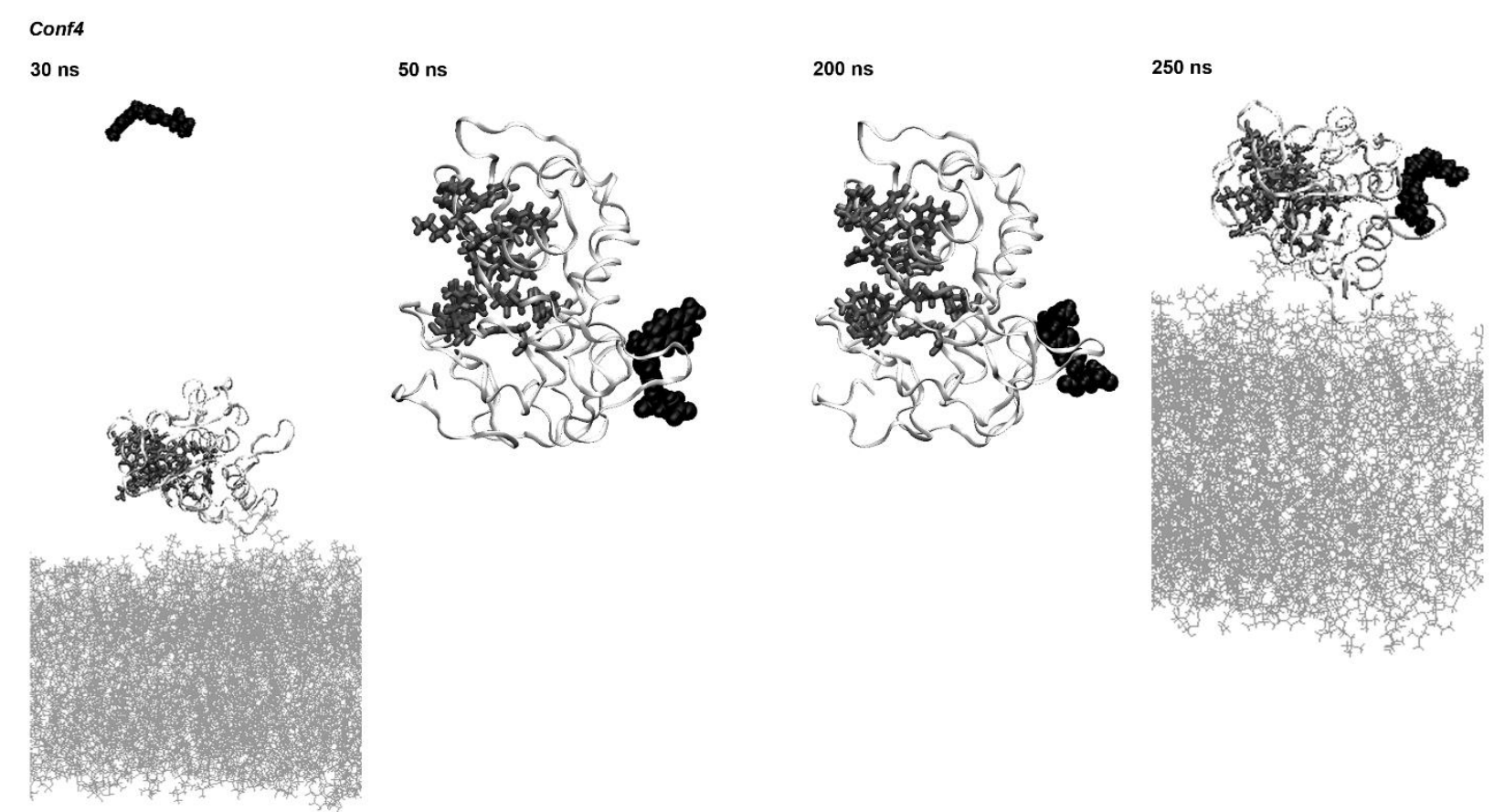

Figure S10: (Continued)

From 158ns on, MQ starts gradually turning and at 160 ns begins entering the pocket interior. This continues smoothly until $165 \mathrm{~ns}$. Then, there is some retreat and MQ remains for some time (up to $178 \mathrm{~ns}$ ) in the middle of the pocket. After that, the ligand continues entering and at the end of the trajectory RTX is completely inserted into the protein cavity.

In Conf2, RTX has three bound periods with respective duration of 39 ns, 62 ns, and min. $42 \mathrm{~ns}$. In the first bound segment, the ligand comes from the solution and binds with MQ to the 'back' of the receptor, opposite the binding site (Figure S10). RTX remains there until 19 ns with the entire molecule spread along the protein surface and interacting with it. Then the ligand moves downward and stays at the base of FR $\alpha$ (far from the GPI) up to $78 \mathrm{~ns}$. MQ is the initial interacting fragment, replaced after that by MAT and Glu. Until $38 \mathrm{~ns}$, FR $\alpha$ is upright relative to the lipid bilayer and the ligand-binding pocket points sideways. Then, the protein starts bending toward the membrane but this does not affect the binding of RTX. The ligand is severely compacted most of the time, which is highly atypical for its structure in unbound state. ${ }^{514}$ At 48 ns RTX retreats from the protein, then shortly comes back to its initial upper position, and at 51.5 ns dissociates and goes to the membrane. In the second bound period, the ligand attaches at the same spot on the surface of FR $\alpha$ from 64 ns on and behaves in a similar way. At 120 ns, RTX starts interacting heavily both with FR $\alpha$ and with the membrane (Figure S10). At $128.7 \mathrm{~ns}$, it dissociates in a manner similar to the first occasion - first stretches out and then floats to the solution. This binding instability may be partially related to the ligand being forced into a strained compact conformation by the particular interactions with the protein in-between the two loops. In the third bound period, the spot of adsorption and the conduct of RTX are analogous to those in the 
first two trajectory segments. The ligand in this case interacts almost exclusively with amino acids from the upper protein loop (Figure S10).

In conf4, FR $\alpha$ is mostly bent toward the membrane with the pocket pointing at it. This may be one of the reasons for the ligand to choose the allosteric site. RTX swims in the solution above the protein and at 38.5 ns contacts with MQ the lower loop at the base of FR (Figure S10). The ligand then rotates and readjusts between the upper and the lower loop, similar to Conf2. The structure of RTX is very compact most of the time, while FR $\alpha$ stands up and bends back to the membrane dynamically (but is predominantly bent). The ligand remains locked between the two loops in the entire bound time with its glutamate pointing to the base of the protein.

\section{Pemetrexed}

PTX adsorbs on top of the protein in Conf1 and Conf2 and contacts the ligand-binding pocket only with its glutamate part. In Conf3 and Conf4 the molecule binds to the same allosteric spot at the bottom of FR $\alpha$ as RTX.

At the beginning of the production trajectory of Conf1 PTX is bound at the top of the pocket (Figure S11) and interacts with Gly137 and Lys136 on one side and with Trp140 on the other. It remains like this until $27 \mathrm{~ns}$ with the molecule upright, glutamate pointing toward the membrane and pyrrolopyrimidine (PP) sticking out above the protein. The whole molecule couples to FR $\alpha$ but only Glu makes closer contact with residues from the pocket. The top loop of the receptor is heavily involved in attracting PTX. At 27 ns the ligand rotates up by $90^{\circ}$ and only PP interacts with the loop. At 29 ns the molecule flings back after severely perturbing locally the secondary structure of FR $\alpha$. PTX remains bound in the initial position until ca. $43 \mathrm{~ns}$. Then it rotates fast (for $\sim 1.5 \mathrm{~ns}$ ) and assumes a position (Figure S11) similar to the initial one of FA (Figure S7, Conf1, 72 ns). At 96 ns PTX turns down and spans the pocket entrance, similar to MTX (Figure S9, Conf4, 130 ns) but oriented oppositely. At 104. 65 ns the ligand rotates by $90{ }^{\circ}$ and stays bound only with glutamate to Lys136 up to $109 \mathrm{~ns}$. It then wobbles a lot and at $112.5 \mathrm{~ns}$ attaches at the back of $F R \alpha$, behind the pocket (Figure 6 of the main manuscript). The ligand is flexible but remains bound to the same spot with at least one of the fragments gradually transferring up. At 143.5 ns PTX comes from behind to the top of the pocket, glutamate interacting with it, mostly with Lys136 (Figure 6). The molecule remains in this position until $238 \mathrm{~ns}$. It then rearranges away from FR $\alpha$ and at 241 ns readsorbs with PP at the top loop. It rotates intensively there and at 248 ns dissociates from the protein and floats to the solution.

In Conf2, PTX comes from the solution above FR $\alpha$ and at 160 ns binds with glutamate to the top loop farther from the pocket. Then it scans the protein surface there and slowly approaches with glutamate the same spot on top of the pocket (Figure 6) as in Conf1. PTX stays tightly bound on top of the protein behind the pocket until 186 ns without changing its position much. Then, it turns by $90^{\circ}$ but remains on top. After that the molecule slowly retreats from the pocket, 'crawling' on the protein surface. 

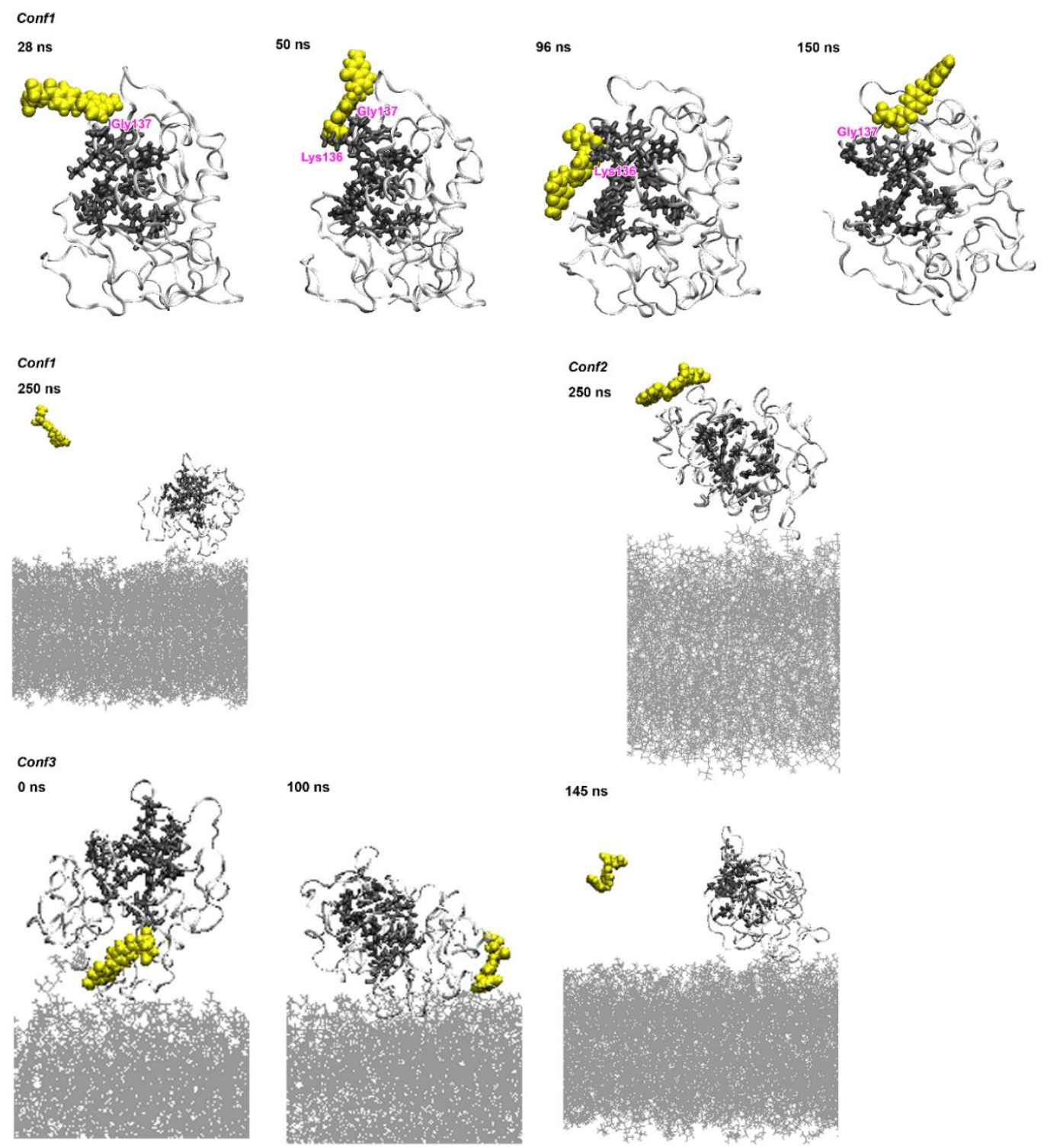

$145 \mathrm{~ns}$
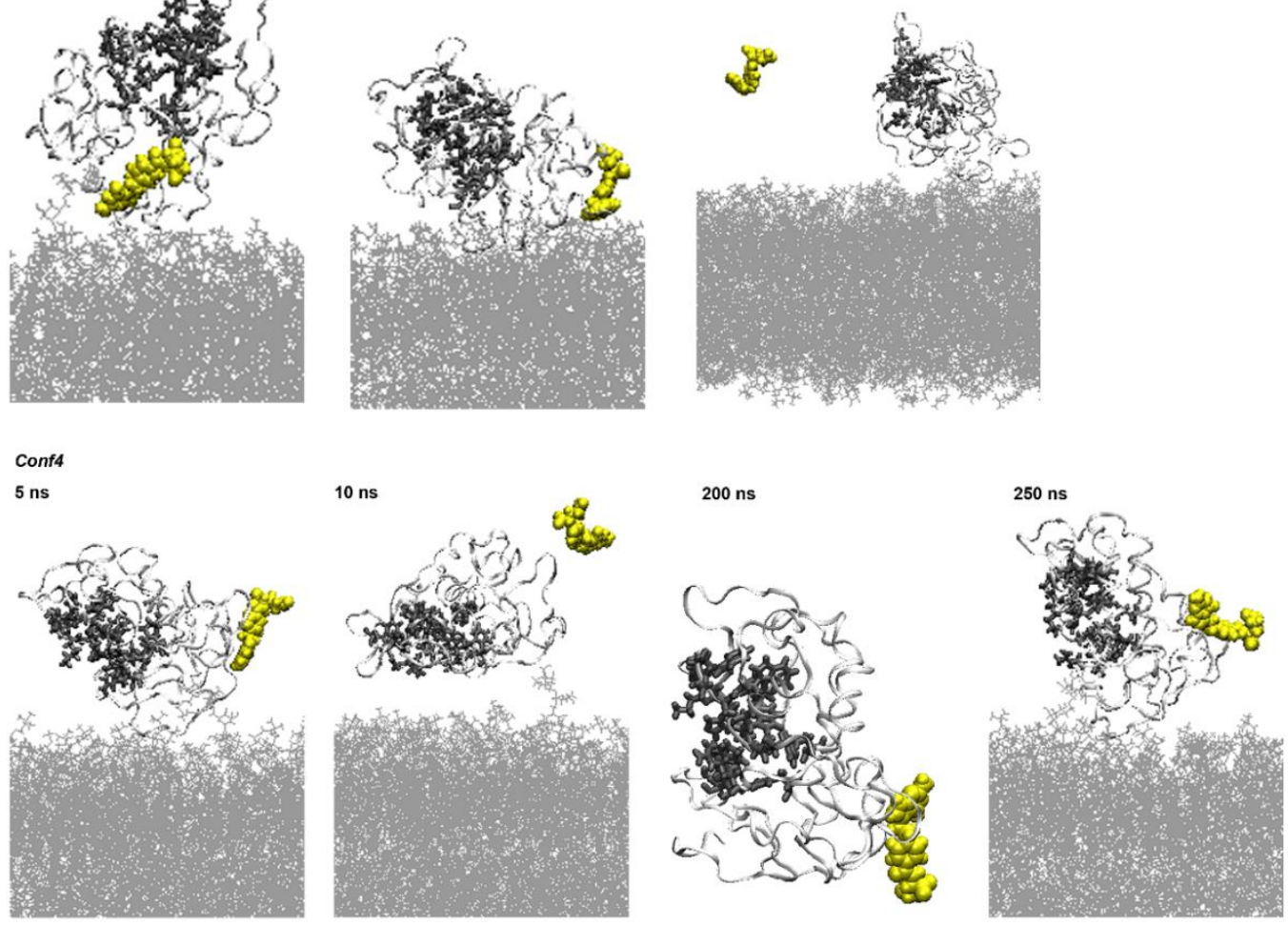

Figure S11: Representative snapshots from the trajectories of PTX illustrating its binding to FR $\alpha$; the membrane lipids are in silver lines, the protein - in white ribbons, its ligand-binding pocket in grey licorice, and PTX - in yellow spheres; water and inorganic ions are omitted for clarity; key interacting amino acids are labeled in magenta 
In this period, it interacts mostly but not exclusively with ethylbenzoate (EB). PTX preserves this behavior up to the end of the trajectory (Figure S11). It should be noted that most of the time the receptor is upright and the pocket is open, i.e., there is no physical barrier for the binding of the ligand into the pocket. It just prefers adsorbing on top of FR $\alpha$.

Already at 0 ns in Conf3 PTX is adsorbed at the base of the protein close to the GPI (Figure S11). It interacts simultaneously with the anchor, with $F R \alpha$, and with the membrane. This lasts until $100 \mathrm{~ns}$. The protein is rather unfolded and the pocket entrance points away from the bilayer in the first half of the period and down to it in the second half. Most of the time PTX lies on the membrane and at the same time PP interacts with the lowest situated loop of FR $\alpha$ (Figure 6, 50 ns) in a manner similar to RTX in Conf2 (Figure S10). In the period 97.5-111.5 ns PTX rotates by 90 ' so that glutamate can concurrently couple to the two loops at the base of the protein (Figure S11). After that the ligand moves forth and back between the two orientations. At 143 ns PTX suddenly dissociates from FR $\alpha$ and floats into the solution.

At the beginning of the trajectory in Conf4 PTX is close to the bottom of the receptor. It rotates a lot until $10 \mathrm{~ns}$ and slides a bit on the protein surface. After that PTX is stably bound at the same allosteric site at the base of the protein (Figure S11). It soon assumes in a very similar orientation to that of RTX in Conf4 (Figure S10) - the molecule is locked between the two bottom loops of FR $\alpha$. It remains like this until the end of the trajectory. The molecule rotates and readjusts without changing its binding spot. Overall, the conduct of PTX is very alike to that in Conf3. The difference is that FR $\alpha$ is mostly bent and the pocket entrance faces the lipid bilayer.

\section{5-Methyltetrahydrofolate}

At the start of the Conf1 trajectory MTHF is already adsorbed on FR $\alpha$ - parallel to the protein surface with glutamate at the bottom of the pocket and tetrahydropteroyl (THP) interacting with the GPI anchor. At $5.5 \mathrm{~ns}$, MTHF rotates by $90^{\circ}$ and orients parallel to the membrane. From 6.25 ns on it spins a lot and moves along the protein surface, mostly scanning its base. At $15 \mathrm{~ns}$, MTHF briefly interacts with the bottom of the pocket entrance with THP. At 18.25 ns it retracts from the pocket, rotates, and buries with THP close to the GPI, where it remains bound from 20 ns on (Figure S12). The glutamate points up, facing the protein, while THP is very close to the membrane and couples to it. The entire ligand interacts heavily with the GPI sugars. MTHF remains like this quite immobilized parallel to the GPI during the entire bound period, i.e., until the end of the trajectory. The ligand-binding pocket points up or sideways (Figure 7) and is open throughout the run. So, the preference of the ligand for the GPI/membrane is not caused by blockade of the active site. 

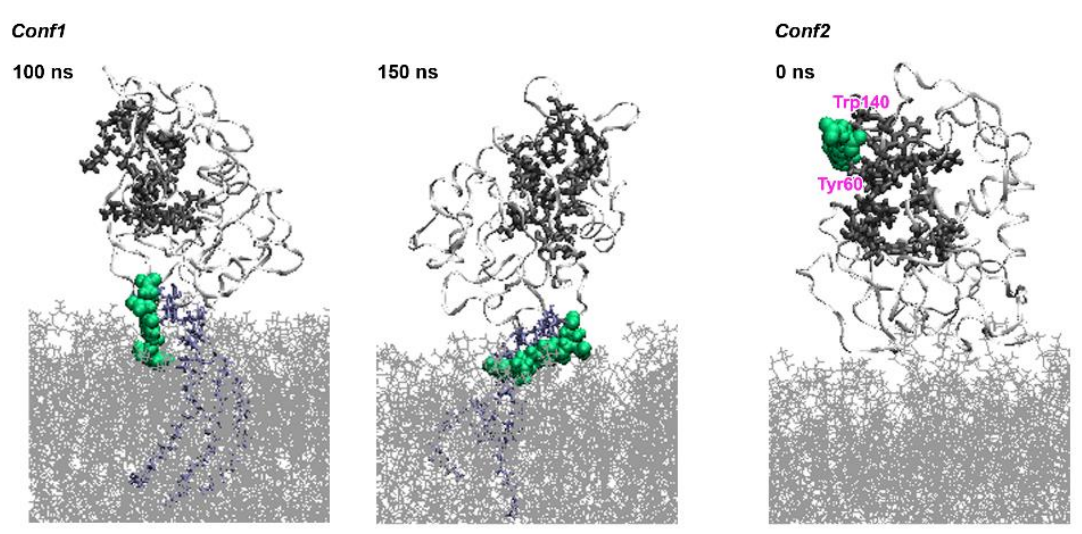

$80 \mathrm{~ns}$
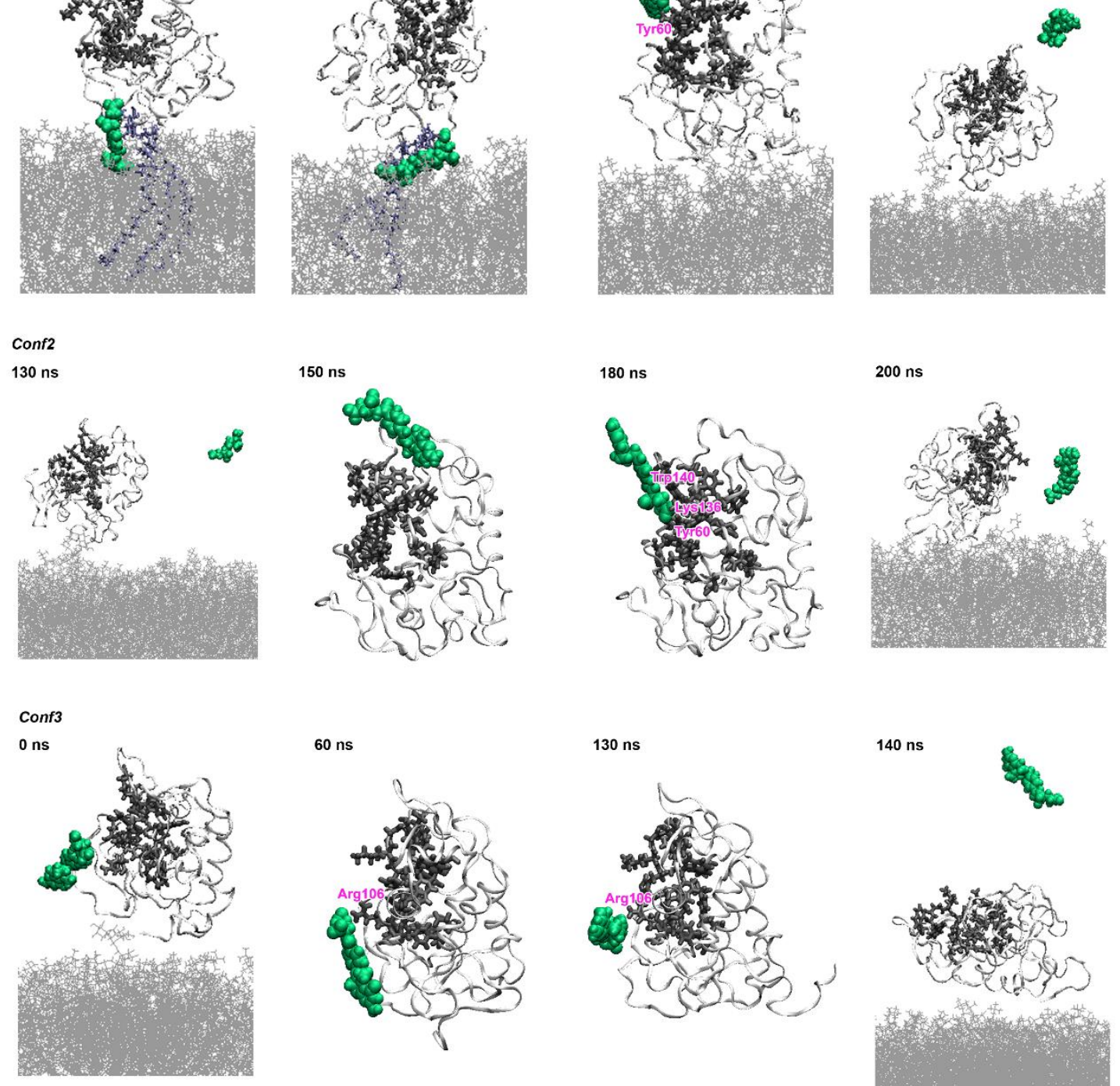

Conf4

$20 \mathrm{~ns}$

$40 \mathrm{~ns}$
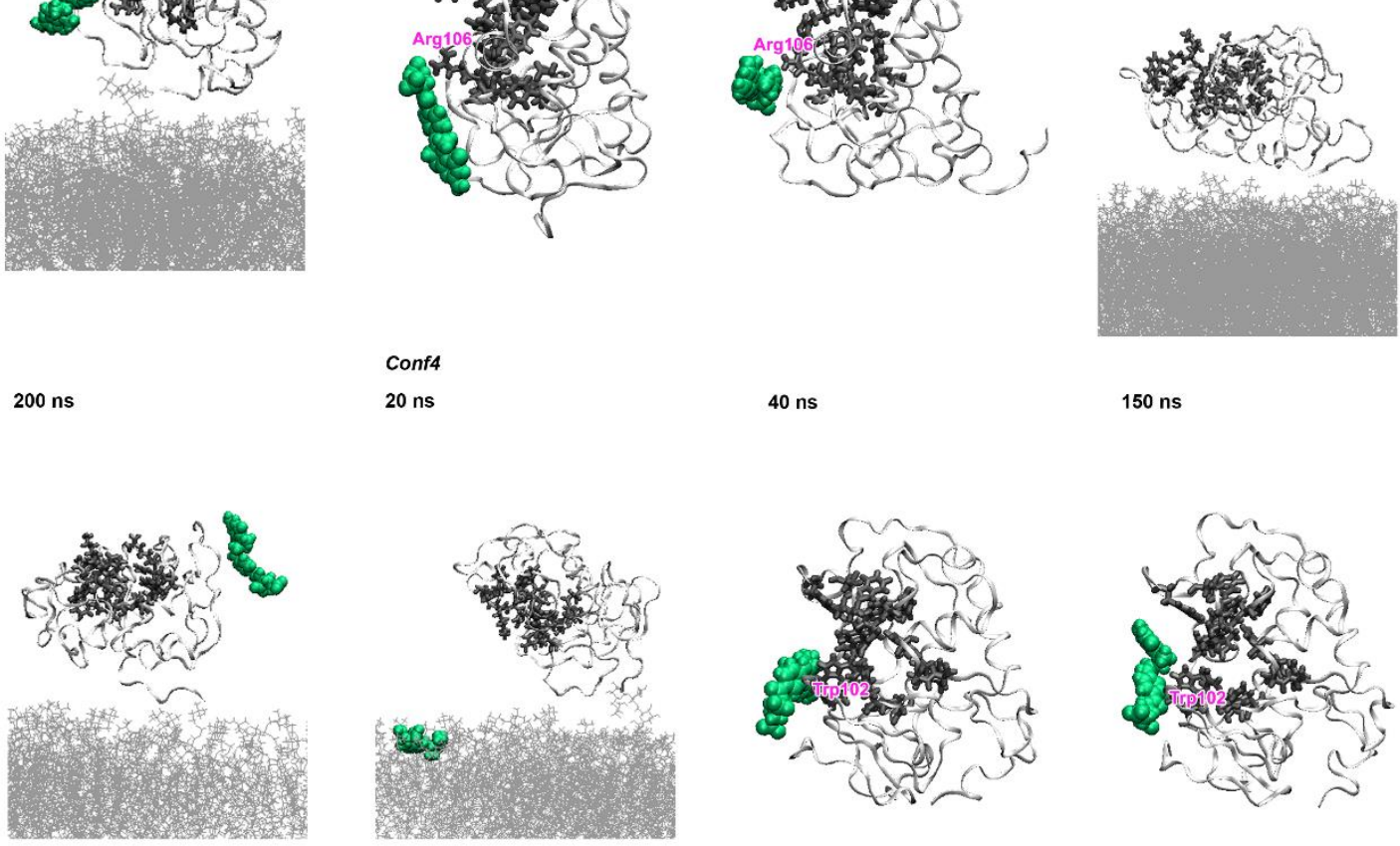

Figure S12: Representative snapshots from the trajectories of MTHF illustrating its binding to FR $\alpha$; the membrane lipids are in silver lines, the protein - in white ribbons, its ligand-binding pocket in grey licorice, GPI - in ice-blue bonds, and MTHF - in green spheres; water and inorganic ions are omitted for clarity; key interacting amino acids are labeled in magenta 

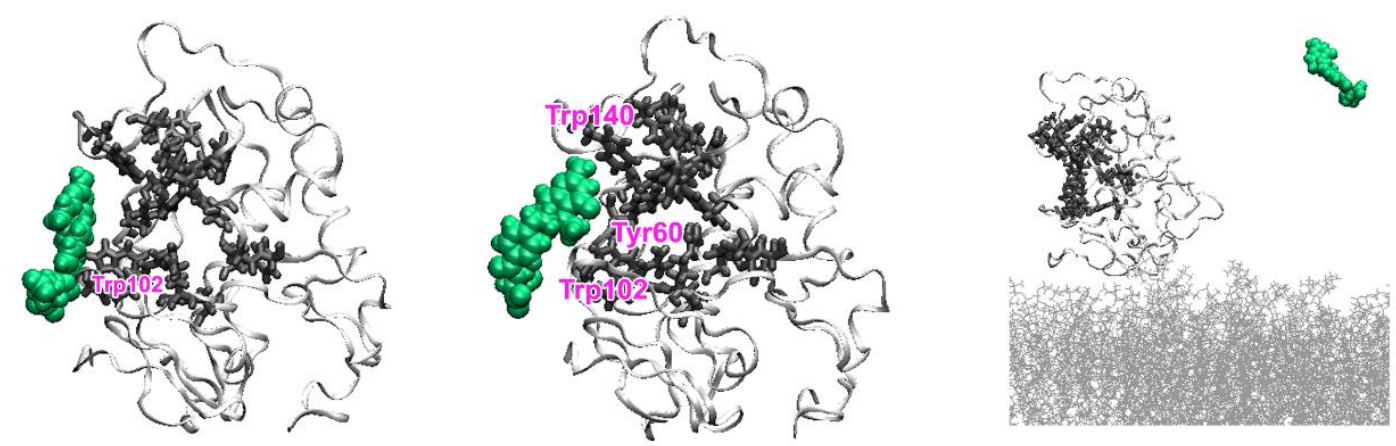

Figure S12: (Continued)

At the beginning of Conf2 MTHF is also bound to FR $\alpha$ but this time at the pocket entrance in a manner very similar to that of FA (Figure S12). However, unlike FA, the aminobenzoate fragment of MTHF is $\pi$-stacked by Trp140 and Tyr60. The ligand remains like this until 53 ns when it suddenly rotates by $90^{\circ}$ and stands with THP pointing up. Shortly after that, it returns and stays bound perpendicularly at the pocket entrance until $77.75 \mathrm{~ns}$. At this moment, the ligand suddenly dissociates and floats to the solution above the protein. At $134 \mathrm{~ns}$, MTHF approaches again FR $\alpha$ from the solution and soon after that binds at the topmost loop (Figure S12). The molecule is fully stretched along the protein and there is no direct interaction with the pocket residues. At $154 \mathrm{~ns}$, glutamate makes contact with Lys136 at the top of the pocket. At 166.75 ns, MTHF binds to the pocket entrance as in the first bound period. However, the binding is not stable this time (it should be noted that the cavity is blocked by Tyr60, Figure S12) and MTHF wanders between the pocket and the top loop, detaching several times from the receptor. Finally, at 198 ns it dissociates permanently and diffuses into the solution.

In the initial moments of the trajectory in Conf3 MTHF is bound with glutamate to the lower end of the pocket entrance. It is attracted mostly by Lys136 and Arg106 (Figure 7) in a way very similar to FA in Conf3 (Figure 4). The rest of the molecule points mostly down to the base of the protein but rotates quite intensely. At $46.5 \mathrm{~ns}$, MTHF detaches from Lys136, moves to the lower end of the pocket entrance (Figure S12) and remains there until $81.5 \mathrm{~ns}$. Then, MTHF goes away from the pocket without desorbing from the surface of FR $\alpha$, slides down, and at 86 ns adsorbs close to the receptor base. After that, it moves up again and from $90 \mathrm{~ns}$ on attaches at one side of the pocket. The ligand rotates and readjusts a lot up to $130 \mathrm{~ns}$, occasionally interacting with pocket residues (primarily Lys136 and Arg106). At $138.5 \mathrm{~ns}$, MTHF simply dissociates into the solution above FR $\alpha$. At 156.5 ns, the ligand comes back but adsorbs at the base of the receptor. It then 'crawls' quickly to the lower side of the pocket entrance, the same as at the beginning of the trajectory. It remains there very shortly, then moves again to the base of FR $\alpha$, unfolds the $\mathrm{N}$ terminus, attaches to it and starts waiving around together with it. The ligand rotates quite 
extensively but this bound configuration lasts until the end of the trajectory. It is noteworthy that the molecule of the receptor is bent toward the membrane all the time but the pocket points up and is accessible.

In Conf4, MTHF is buried almost entirely into the membrane after $10 \mathrm{~ns}$ (Figure S12). At $28 \mathrm{~ns}$, it exits and goes into the solution, where it 'swims' freely. At $37 \mathrm{~ns}$, the ligand approaches very fast the protein and adsorbs with glutamate at the pocket entrance close to Trp102. It remains there adjusting dynamically, sometimes stretched along the cavity entrance, up to 235 ns. Sometimes the molecule orients at the entrance very similar to FA but does not bind that tightly at all. Tyr60 blocks the cavity volume in this trajectory as well (Figure S12, snapshot at 235 ns). Overall, in this binding position MTHF interacts mainly with Trp102 and Arg61 with its THP and aminobenzoate fragments. Most of the time glutamate hangs down well below the pocket (Figure 7). At $235 \mathrm{~ns}$, MTHF tries to enter the pocket with THP. However, the attempt is unsuccessful because Tyr60 still blocks the cavity. Probably that is why at 240 ns MTHF dissociates from the protein and floats into the solution above, locating itself close to the inner leaflet of the lipid bilayer at the end of the trajectory.

\section{Pteroyl ornithine}

PON binds to the protein only in Conf1 and dissociates $90 \mathrm{~ns}$ after that. The ligand floats in the solution most of the simulation time. At 110 ns in Conf1 it approaches the protein shortly but away from the pocket. Then it scans very dynamically the vicinity of the $\mathrm{N}$-terminus and the base of FR $\alpha$. The ligand detaches again and at 125 ns finally adsorbs at the back of the protein, opposite the binding pocket (Figure S13).

Pterin interacts most with the receptor and this continues until $185 \mathrm{~ns}$. After that, also ornithine starts making short contacts with FR . At 220 ns PON dissociates briefly and then readsorbs higher on the protein surface (Figure S13). It detaches from there at $225 \mathrm{~ns}$ and returns at $227.5 \mathrm{~ns}$ even higher but on the same side of the protein. The ligand then scans actively the receptor surface with pterin, rotating a lot, until 246.25 ns when PON dissociates permanently and approaches the membrane. It should be noted that the ligand does not interact with the binding pocket at all. This deteriorated affinity of pteroyl ornithine for FR $\alpha$ stems from the replacement of the dianionic glutamate with the zwitterionic ornithine, which underlines the importance of the electrostatic attraction for the targeting ability of folate-based ligands. 


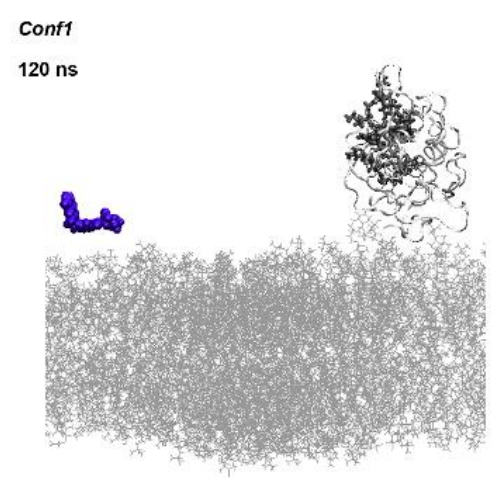

$130 \mathrm{~ns}$

$150 \mathrm{~ns}$

$200 \mathrm{~ns}$
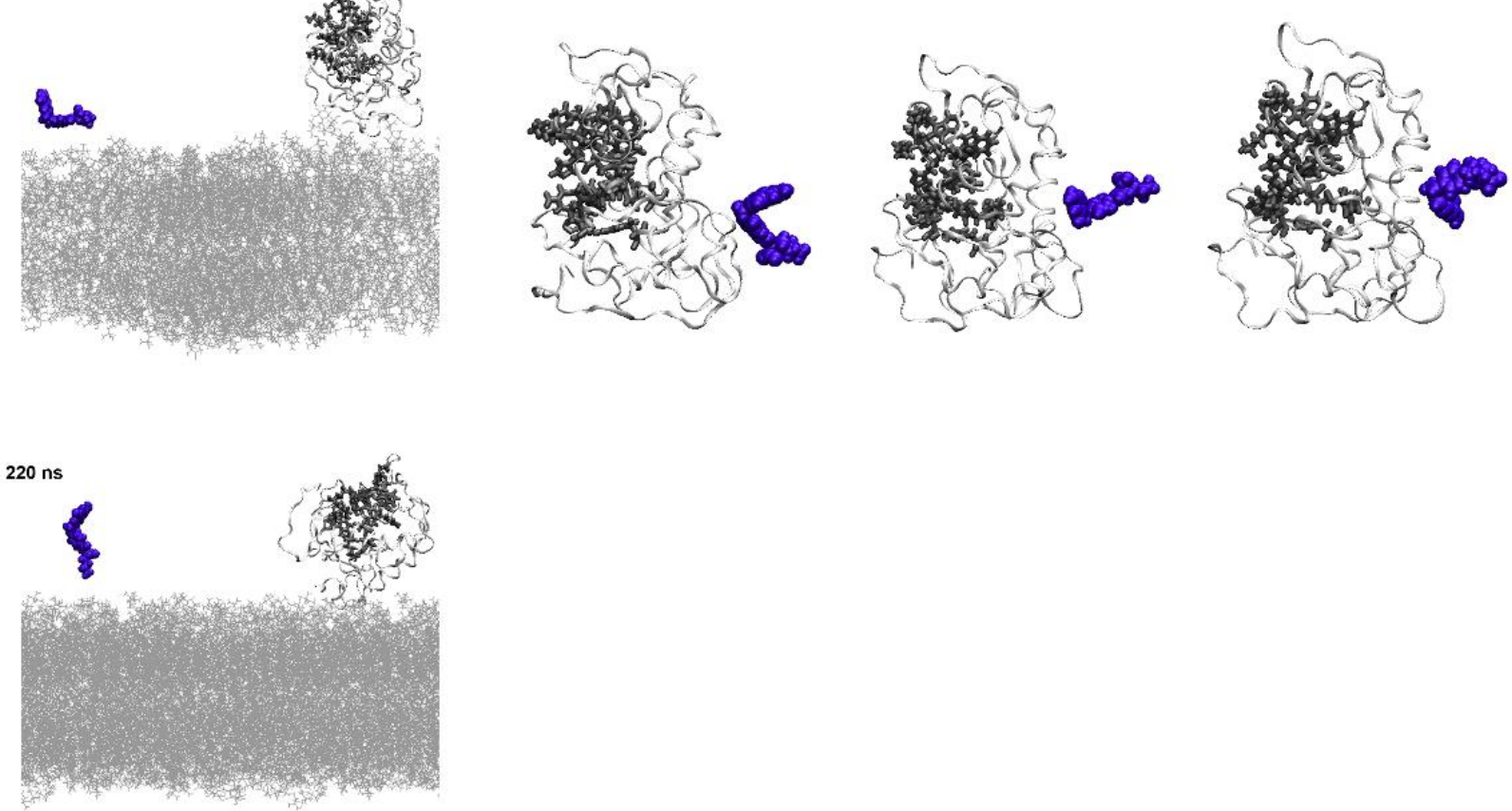

Figure S13: Representative snapshots from Conf1 of PON illustrating its adsorption on the surface of $F R \alpha$; the membrane lipids are in silver lines, the protein - in white ribbons, its ligand-binding pocket - in grey licorice, and PON - in violet spheres; water and inorganic ions are omitted for clarity 


\section{Specific ligand-receptor interactions}

The analyses involving averaging are made for segments of 50 ns from the bound periods of each ligand in the four trajectories. Whenever interactions between some fragments of a ligand and the active site of the protein are analyzed, the amino acids from the latter are those in contact with the particular ligand fragment outlined in the crystallographic structure. ${ }^{\mathrm{s}}$

\section{Folate}

FA couples most often to $F R \alpha$ with the aminobenzoate and with the glutamate part. Hence, only the interactions of these two fragments with the relevant parts of the protein (mostly with the residues from the ligand-binding pocket) are discussed. No sizeable interactions of pterin with its target amino acids from the binding pocket are registered in any of the bound periods: the minimum distance of pterin to them is ca. $1 \mathrm{~nm}$.

Figures S14 to S20 and Table S3 summarize the data from the analyses of the FA models. It is evident from the plots in Figures S14 and S15 that in Conf1 to Conf3 the aminobenzoate and the glutamate fragments of folate interact with their crystallographic partner amino acids from the receptor. Together with the lack of specific interactions of pterin, this verifies that FA binds to the active site of the protein, maintaining most of the key interactions, but orienting in a different manner to the receptor than in the X-ray geometry.

The strength of the specific interactions depends materially on the particular alignment of the ligand at the pocket entrance, which is different in the four trajectories. Most profound coupling of aminobenzoate and glutamate to their target amino acids is witnessed in Conf1 (Figures S14, S15). Moreover, the interactions intensify over time. There is some dynamic rearrangement of FA in the period 200-250 ns and after that, a steady contact with the pocket amino acids is maintained. The stabilizing interactions are a combination of glutamate-backbone coupling to Gly137, His135, and Trp138, dispersion attraction of aminobenzoate mostly to Trp140 (Figure S17), Trp102, and Tyr60 (Figure S15), and specific hydrogen bonds combined with nondirectional electrostatics between the dianionic glutamate and Lys136 and Trp140 (Table S3, Figure S16). The same interactions of folate are present also in Conf2 but all of them are much less expressed. The more intensive dynamics of the ligand in the binding site in this trajectory is evidenced by the much lower and shallower RDF maxima of glutamate and the much smaller average number of its hydrogen bonds. This is supplemented by the occasional loss of contacts of aminobenzoate with Trp102 and Tyr60, and by the frequent exchange of pterin and $A B$ as preferred closest partners of Trp140.

All these interactions are even less expressed in Conf3, which might explain why the ligand dissociates from the pocket in this trajectory. The specific electrostatic stabilization of FA there is illustrated by the RDFs and hydrogen bonds with Arg106 and Lys136.

The RDF maxima in Conf3 are well shaped over the entire bound period (Figure S14) with preferred distance $<0.5 \mathrm{~nm}$ and the 'clamp' of glutamate is stabilized by 2 to 3 hydrogen bonds 
with Arg106 and 1 to $2 \mathrm{H}$-bonds with Lys136 (Table S3). It is evident that the coupling, however, is weaker in the second half of the bound period. Then, the H-bonds with Arg106 are broken at ca. $115 \mathrm{~ns}$, followed by the same loss of binding with Lys136 at ca. $118 \mathrm{~ns}$, which eventually causes the unbinding of the ligand.

The particular behavior of folate in Conf4 is accompanied also by varying interactions. In the second bound period (80-138 ns), when the ligand is close to the pocket, there is some stabilization by weak but persistent interaction of AB with Trp140 (Figure S17). The main contributions, however, are coupling to Arg61 both by electrostatic and dispersion attraction (Figure S18, bottom) and 1 to 3 hydrogen bonds between the glutamate of FA and guanidinium (Figure S19). In the first and third bound period, the coupling of folate to FR $\alpha$ may be characterized as non-specific adsorption. In the segment 20-60 ns the ligand is briefly attracted to the GPI (Figure S18, top), while between 200 and 240 ns it 'scans' the surface of the protein opposite to the binding pocket, thereby interacting directly with 12 different amino acids (Figure S20). 


\section{Configuration 1}
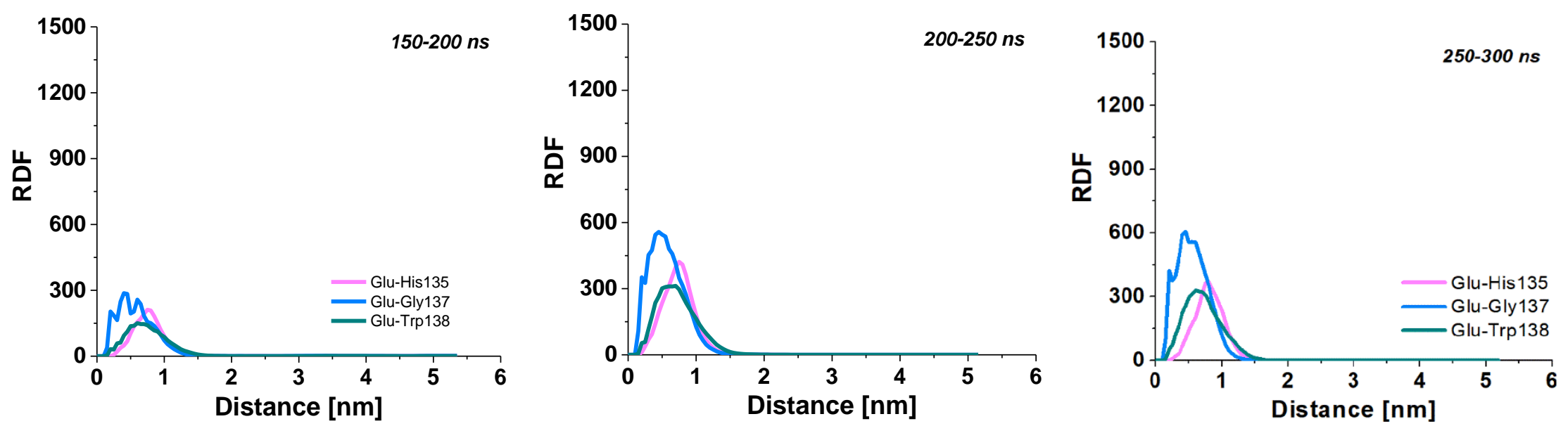

\section{Configuration 2}
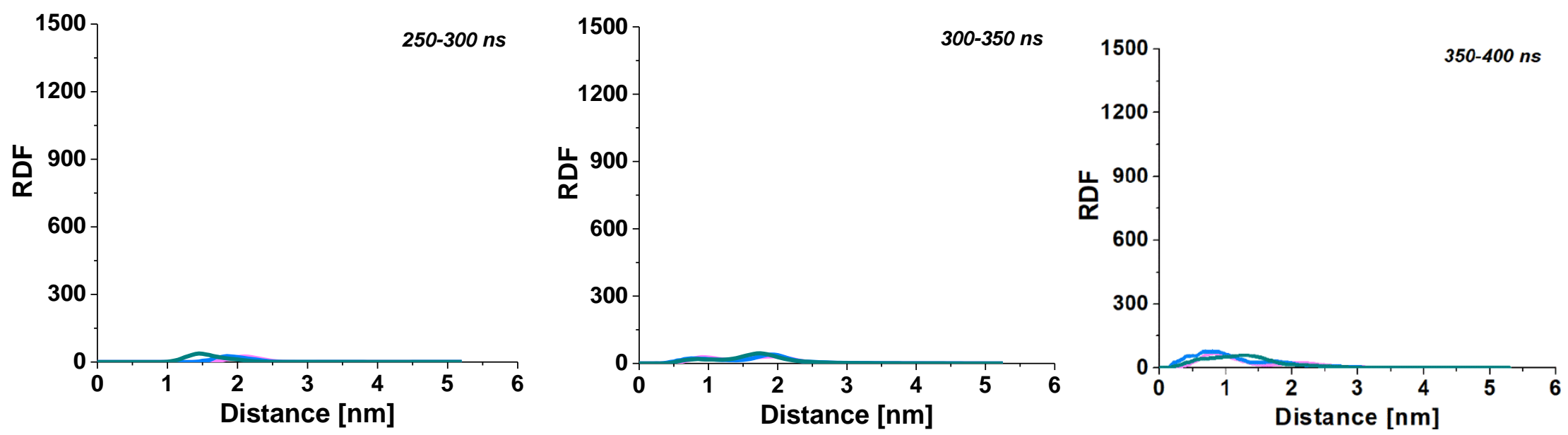


\section{Configuration 3}
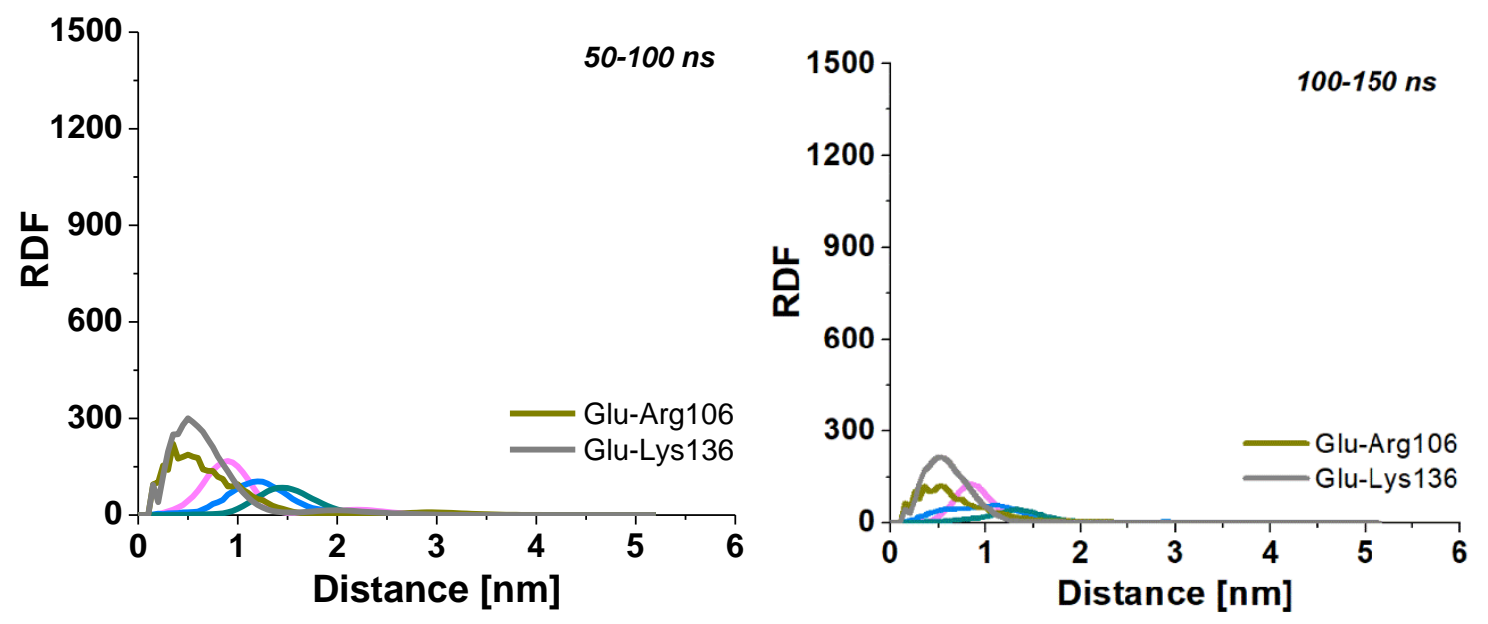

Figure S14: Radial distribution functions of the distance between the glutamate part of folate and its target interaction partners from FRa during the bound periods of Conf1 to Conf3 


\section{Configuration 1}
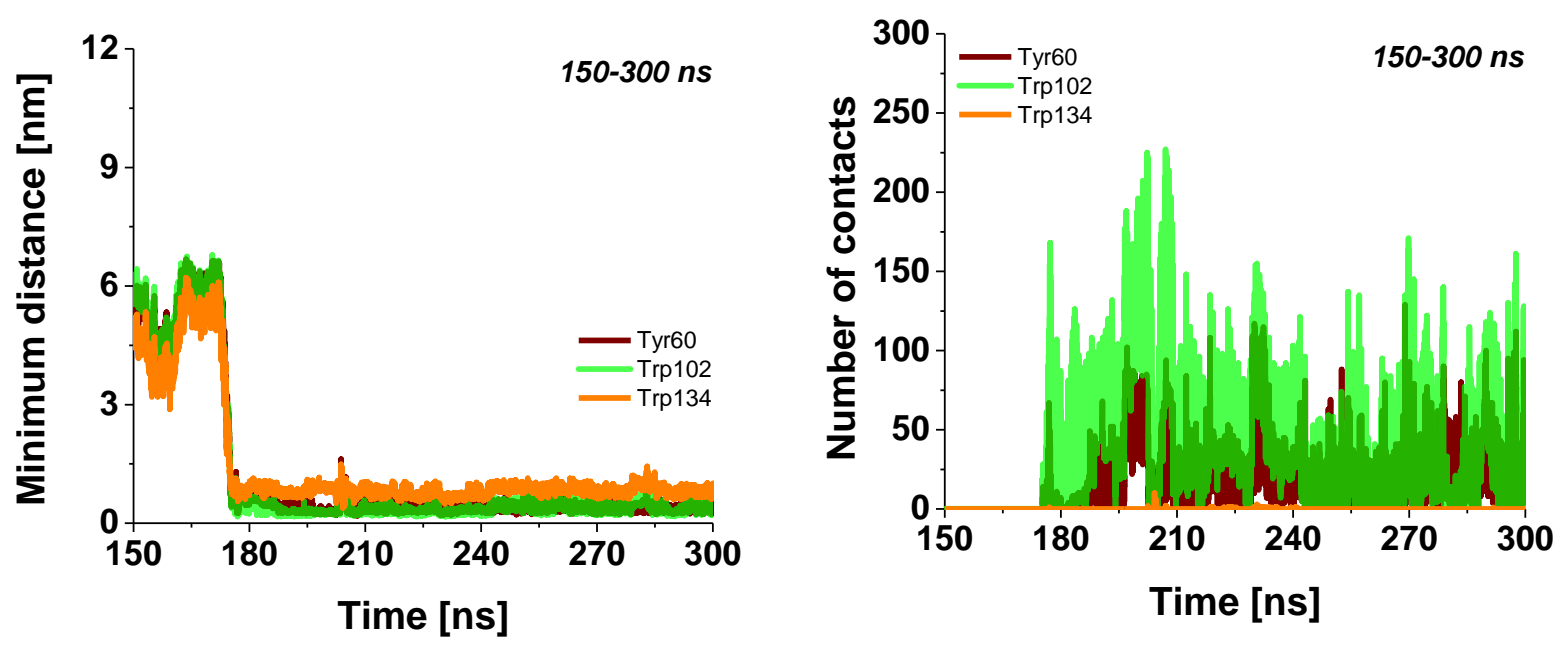

\section{Configuration 2}
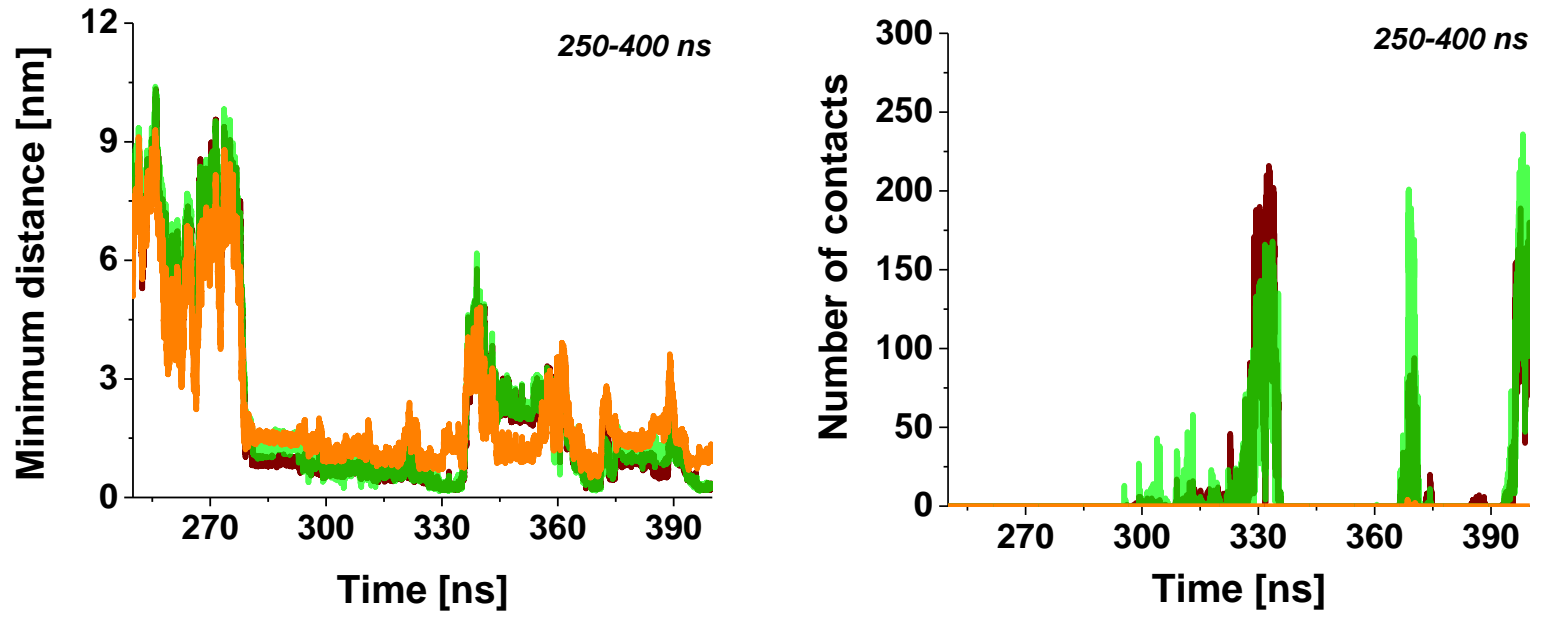

\section{Configuration 3}
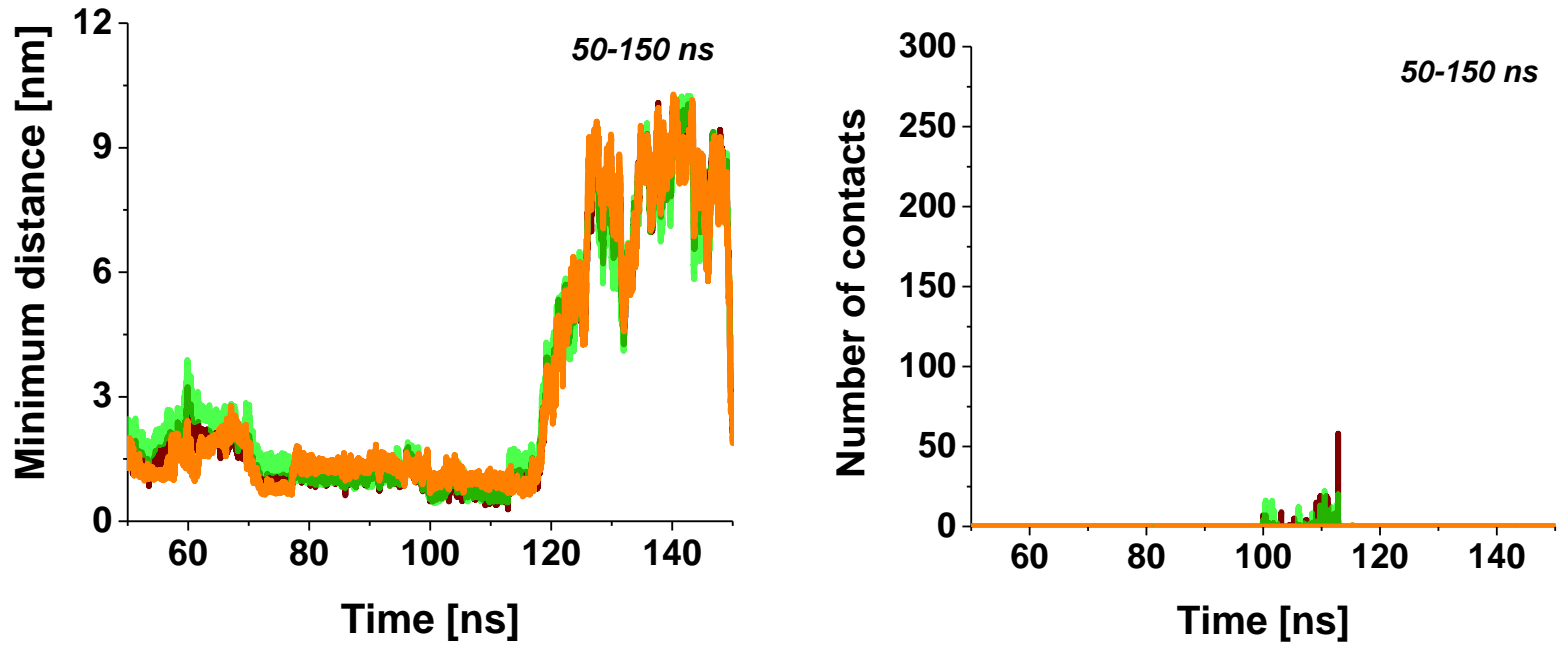

Figure S15: Evolution of the (left) minimum distance and (right) number of contacts during the entire bound periods between atom pairs from the aminobenzoate of FA and its target interaction partners from FR $\alpha$ in Conf1 to Conf3 
Table S3: Average number $\left(\mathrm{N}_{\mathrm{HB}}{ }^{\text {ave }}\right)$ with standard deviation, preferred length $\left(\mathrm{R}_{\mathrm{HB}}{ }^{\text {ave }}\right)$, and type of the hydrogen bonds formed between the glutamate of FA and its target interaction partners from FR $\alpha$ during the bound periods of Conf1 to Conf3

\begin{tabular}{|c|c|c|c|c|}
\hline Amino acid & Period & $\mathrm{N}_{\mathrm{HB}}{ }^{\text {ave }}$ & $\mathrm{R}_{\mathrm{HB}}{ }^{\text {ave }}, \mathrm{nm}$ & Bond type \\
\hline \multicolumn{5}{|c|}{ Configuration 1} \\
\hline \multirow{3}{*}{ Trp102 } & $150-200 \mathrm{~ns}$ & $0.003 \pm 0.051$ & $0.218 / 0.238$ & \multirow{3}{*}{$\alpha$-or $\gamma$-carboxylate group } \\
\hline & $200-250 \mathrm{~ns}$ & $0.001 \pm 0.032$ & 0.228 & \\
\hline & $250-300 \mathrm{~ns}$ & $0.000 \pm 0.014$ & 0.213 & \\
\hline \multirow{3}{*}{ Lys136 } & $150-200 \mathrm{~ns}$ & $0.043 \pm 0.209$ & 0.168 & \multirow{3}{*}{ both carboxylate groups } \\
\hline & $200-250 \mathrm{~ns}$ & $0.402 \pm 0.501$ & 0.168 & \\
\hline & $250-300 \mathrm{~ns}$ & $0.308 \pm 0.541$ & 0.168 & \\
\hline \multirow{3}{*}{ Trp140 } & $150-200 \mathrm{~ns}$ & $0.255 \pm 0.436$ & 0.188 & \multirow{3}{*}{$\gamma$-carboxylate group } \\
\hline & $200-250 \mathrm{~ns}$ & $0.148 \pm 0.356$ & 0.193 & \\
\hline & $250-300$ ns & $0.265 \pm 0.442$ & 0.188 & \\
\hline \multicolumn{5}{|c|}{ Configuration 2} \\
\hline \multirow{3}{*}{ Trp102 } & $250-300 \mathrm{~ns}$ & $0.000 \pm 0.000$ & ----- & \multirow{3}{*}{$\gamma$-carboxylate group } \\
\hline & $300-350 \mathrm{~ns}$ & $0.000 \pm 0.000$ & ----- & \\
\hline & $350-400$ ns & $0.003 \pm 0.053$ & $0.193 / 0.208$ & \\
\hline \multirow{3}{*}{ Lys136 } & $250-300 \mathrm{~ns}$ & $0.000 \pm 0.000$ & $\begin{array}{l}---- \\
--\end{array}$ & \multirow{3}{*}{ both carboxylate groups } \\
\hline & $300-350 \mathrm{~ns}$ & $0.043 \pm 0.217$ & 0.168 & \\
\hline & $350-400 \mathrm{~ns}$ & $0.121 \pm 0.346$ & 0.163 & \\
\hline \multirow{3}{*}{ Trp140 } & $250-300 \mathrm{~ns}$ & $0.000 \pm 0.000$ & ---- & \multirow{3}{*}{$\alpha$-carboxylate group } \\
\hline & $300-350$ ns & $0.017 \pm 0.129$ & 0.193 & \\
\hline & $350-400 \mathrm{~ns}$ & $0.014 \pm 0.122$ & 0.183 & \\
\hline \multicolumn{5}{|c|}{ Configuration 3} \\
\hline \multirow{2}{*}{ Trp102 } & $50-100 \mathrm{~ns}$ & $0.000 \pm 0.000$ & ----- & \\
\hline & $100-150$ ns & $0.000 \pm 0.000$ & ----- & \\
\hline \multirow{2}{*}{ Lys136 } & $50-100 \mathrm{~ns}$ & $0.399 \pm 0.640$ & 0.168 & \multirow{2}{*}{ both carboxylate groups } \\
\hline & $100-150 \mathrm{~ns}$ & $0.209 \pm 0.424$ & 0.173 & \\
\hline \multirow{2}{*}{ Trp140 } & $50-100 \mathrm{~ns}$ & $0.000 \pm 0.000$ & $\begin{array}{l}---- \\
--\end{array}$ & \\
\hline & $100-150$ ns & $0.000 \pm 0.000$ & ----- & \\
\hline
\end{tabular}




\begin{tabular}{|l|l|l|l|l|}
\hline \multirow{2}{*}{ Arg106 } & 50-100 ns & $0.804 \pm 0.998$ & 0.173 & \\
\cline { 2 - 4 } & $100-150 \mathrm{~ns}$ & $0.468 \pm 0.853$ & 0.173 & $\begin{array}{l}\text { both carboxylate groups in the } \\
\text { first period, only } \alpha- \\
\text { carboxylate group in the } \\
\text { second one }\end{array}$ \\
\hline
\end{tabular}




\section{Configuration 1}
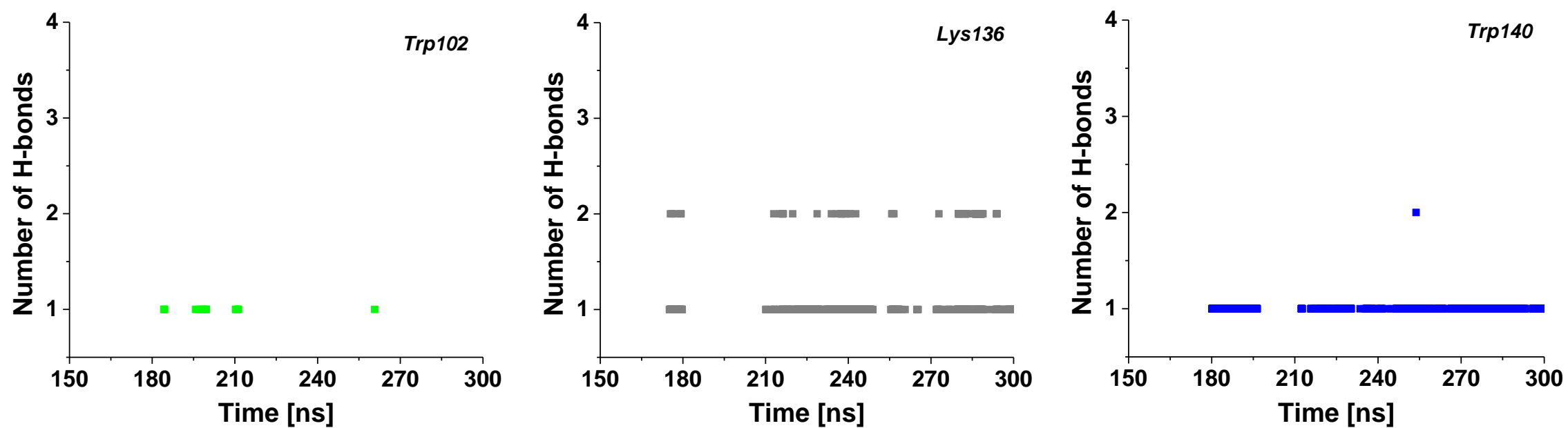

\section{Configuration 2}
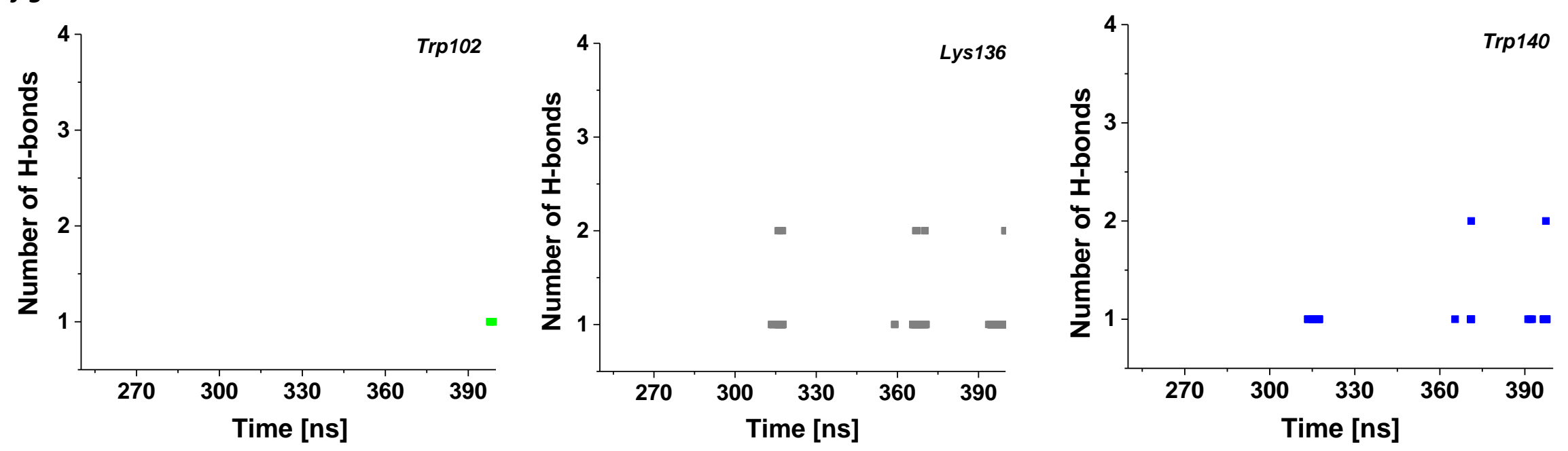

Figure S16: Evolution of the number of hydrogen bonds between the glutamate of FA and its target interaction partners from the binding pocket of FR $\alpha$ during the bound periods of Conf1 to Conf3 


\section{Configuration 3}
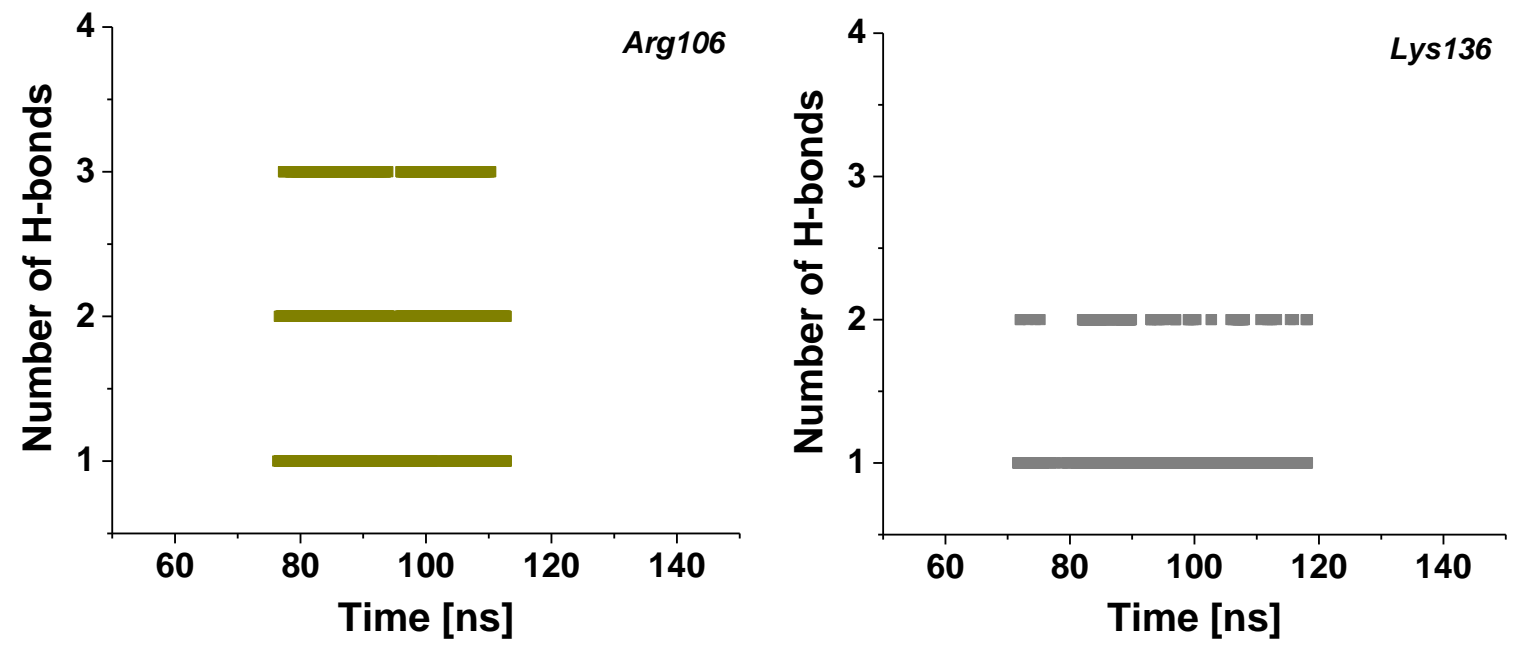

Figure S16: (Continued); No hydrogen bonds were formed between glutamate and Trp102 and Trp140 in Conf3 


\section{Configuration 1}
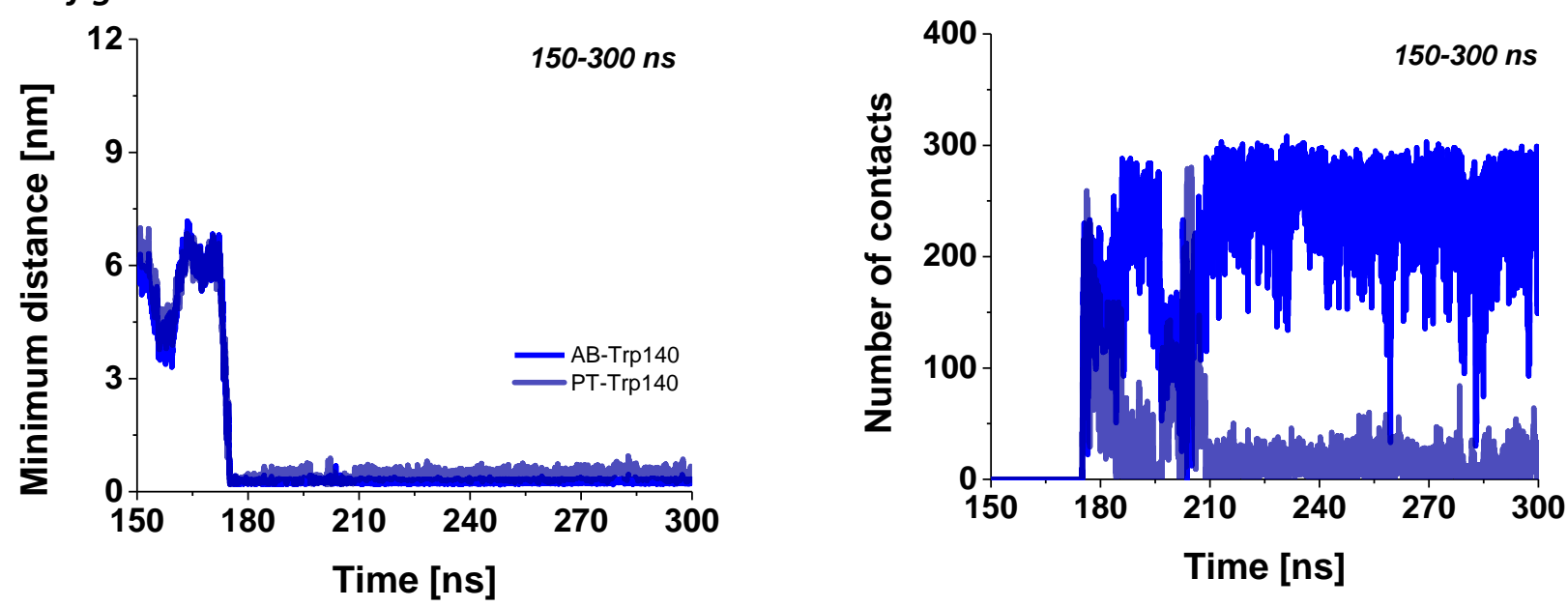

\section{Configuration 2}

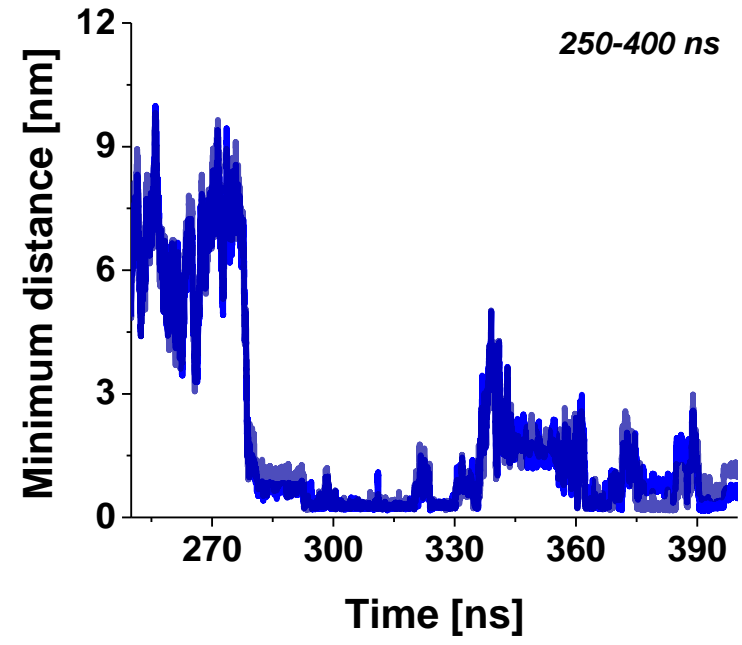

\section{Configuration 3}
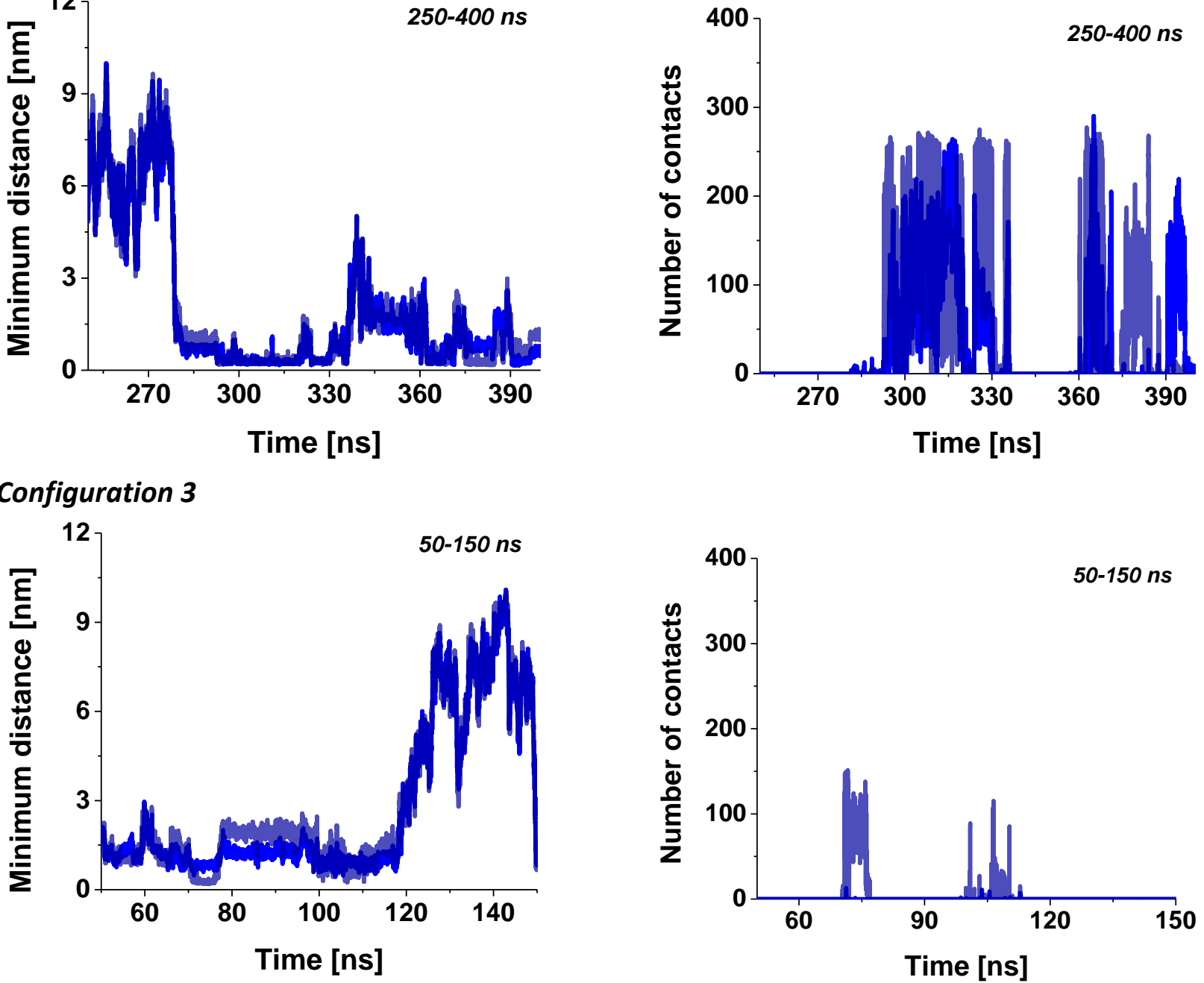

Configuration 4

Figure S17: Evolution of the (left) minimum distance and (right) number of contacts during the entire bound period between atom pairs from the aminobenzoate or pterin of FA and Trp140 from $F R \alpha$ in the four MD trajectories

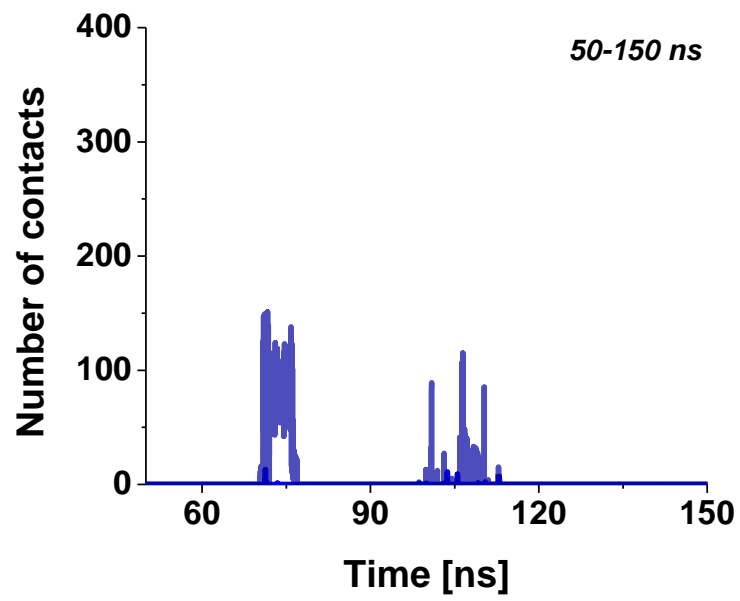




\section{Configuration 4}
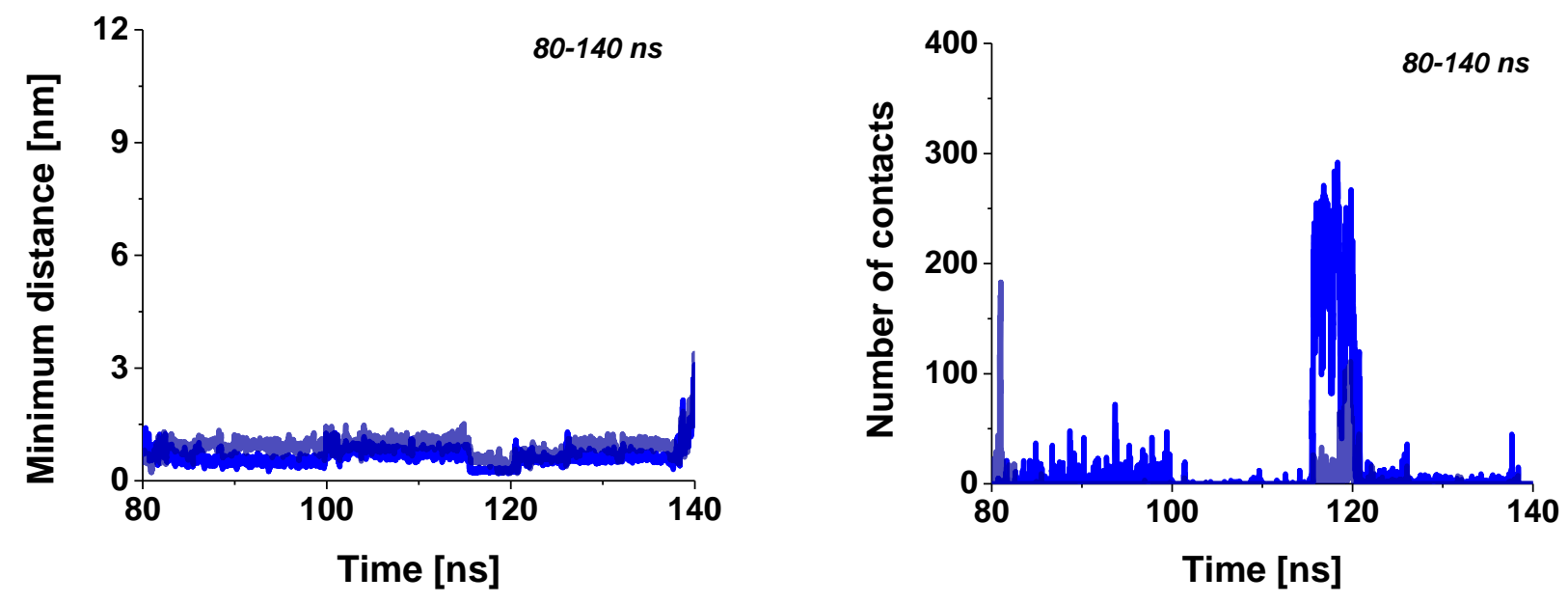

Figure S17: (Continued)

\section{Configuration 4}
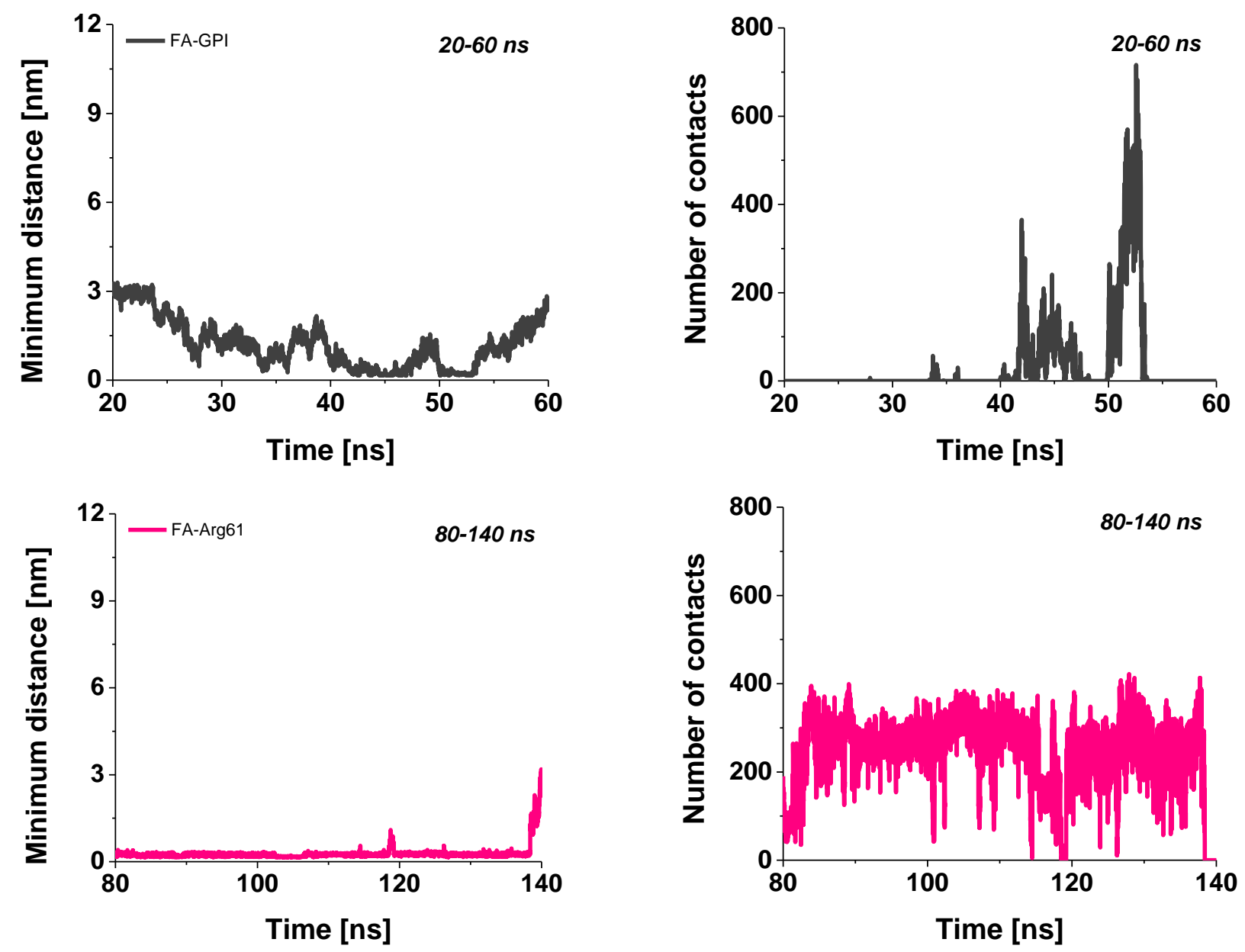

Figure S18: Evolution of the (left) minimum distance and (right) number of contacts between atom pairs from (top) FA and GPI during the first bound period and (bottom) FA and Arg61 during the second bound period of Conf4 


\section{Configuration 4}

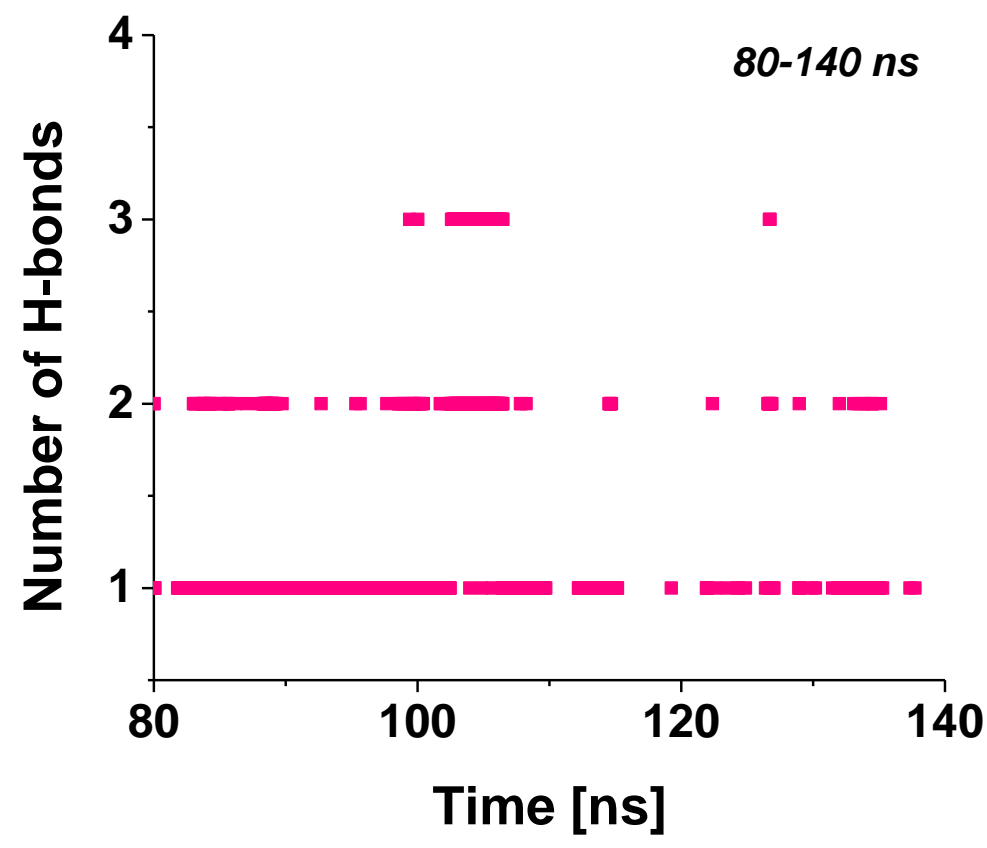

Figure S19: Evolution of the number of hydrogen bonds formed between FA and Arg61 of FR $\alpha$ during the second bound period of Conf4

\section{Configuration 4}
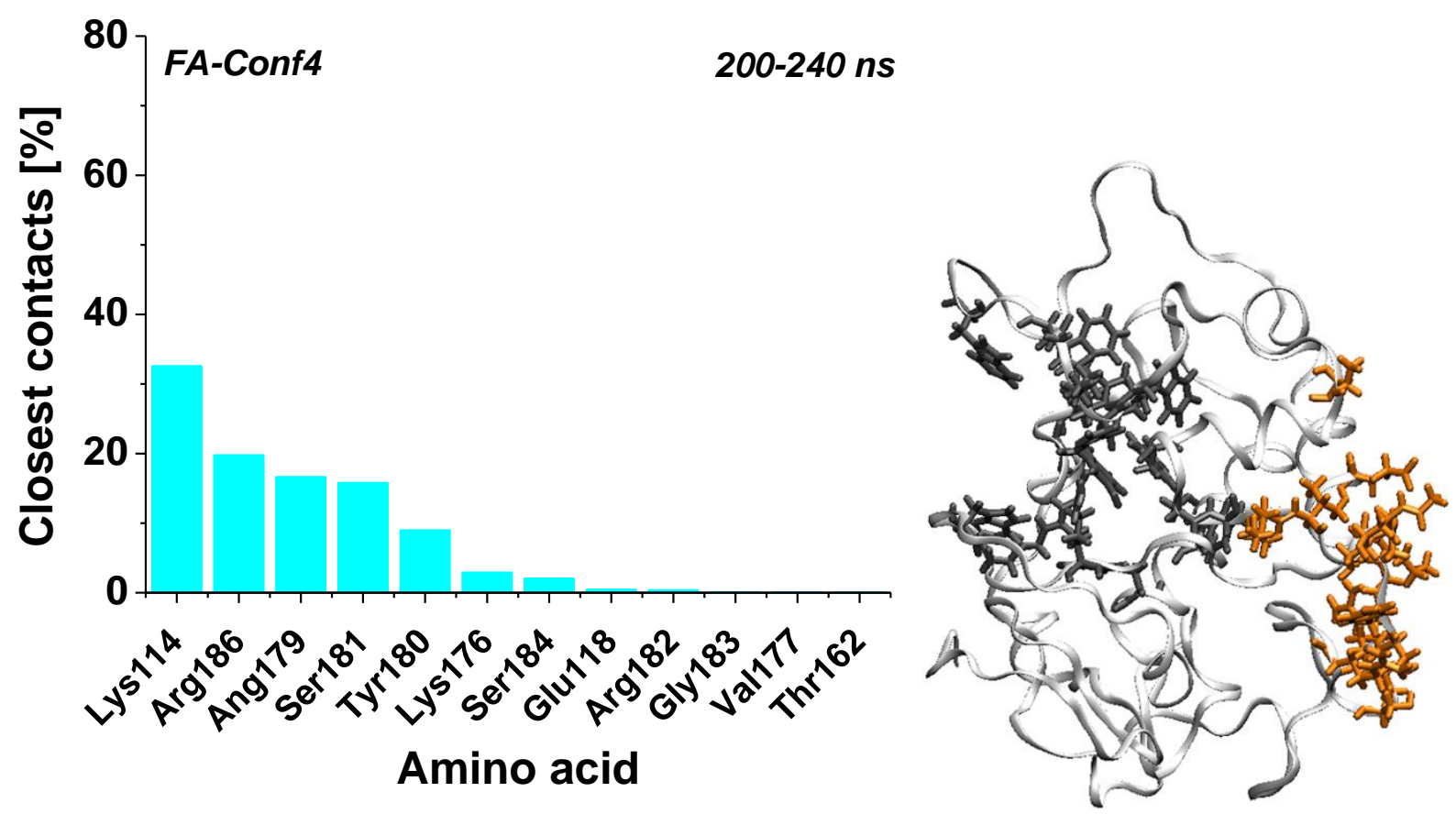

Figure S20: (left) Distribution of the number of minimum distances closed between FA and amino acids from FR $\alpha$ during the third bound period of Conf4 and (right) illustration of these interaction partners on the structure of the receptor at 200 ns; the protein is shown as ribbons, the binding pocket residues - as grey licorice, the interaction partners of FA - as orange licorice 


\section{Methotrexate}

In Conf2, MTX scans the entire width of the receptor at its base (Figure S21), being in close contact with 14 different amino acids. This positioning of MTX is stabilized predominantly by a combination of electrostatic attraction and hydrogen bonding of its anionic glutamate part. Evidently, these interactions are not sufficient to fix permanently the ligand at this spot on the protein surface and it dissociates at $160 \mathrm{~ns}$.

In Conf4, MTX interacts with amino acids from the FR $\alpha$ binding site but aligns mostly perpendicular to the plane of the pocket entrance. This is enabled by pure electrostatic attraction between the glutamate of MTX and Trp140 from FR (without the supplementing hydrogen bond needed for specific attachment, Figure S22, bottom) and simultaneous $\pi$-stacking of amino pterin from MTX and Trp102 from the receptor (Figure S23, top). The latter is by far the dominant intermolecular interaction, which is reinforced occasionally by one hydrogen bond between the amino group bound at C4 of pterin and the sidechain NH group of Trp102 (Figure S23, bottom). This pattern of coupling of MTX to FR $\alpha$ lacks most of the specific interactions characteristic for FA (Figure S22 compared to Figures S14 to S16), which explains why MTX does not attach permanently to the receptor. 


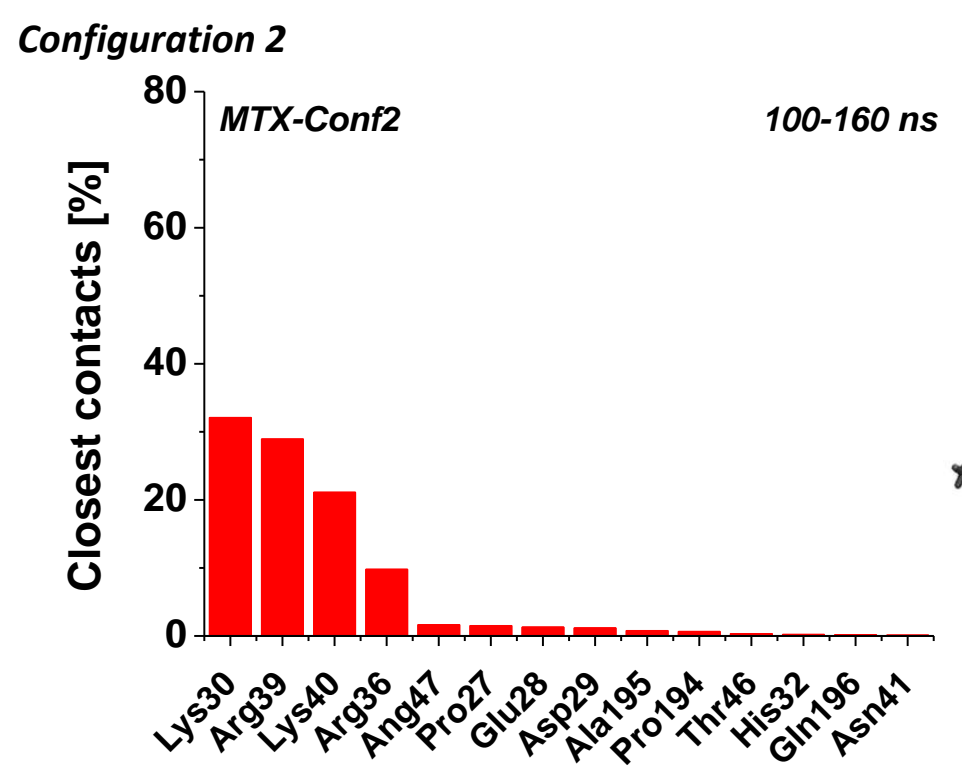

Amino acid

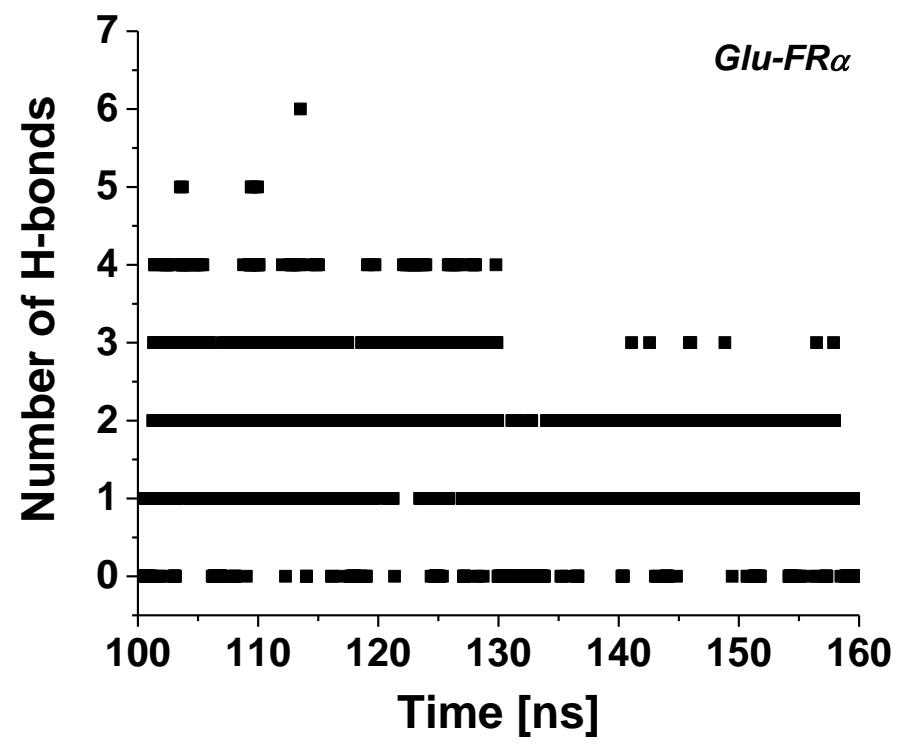

Figure S21: (top left) Distribution of the number of minimum distances closed between MTX and amino acids from FR $\alpha$ during the bound period of Conf2 with (top right) illustration of these interaction partners on the structure of the receptor at $150 \mathrm{~ns}$; the protein is shown as ribbons, the binding pocket residues - as grey licorice, the interaction partners of MTX - as orange licorice; (bottom) evolution of the number of hydrogen bonds of the glutamate of MTX with amino acids from the receptor in the same period of Conf2

In $92 \%$ of the bound time the molecule is in nearest proximity to two Lys and two Arg residues (all them with positively charged side chains). In addition, the glutamate fragment forms between one and six hydrogen bonds throughout the entire trajectory, albeit 48 different $\mathrm{H}$-bonds with 7 different amino acids (Asp29, Lys30, Arg36, Arg39, Lys40, Asn41, and Ala 195). 


\section{Configuration 4}
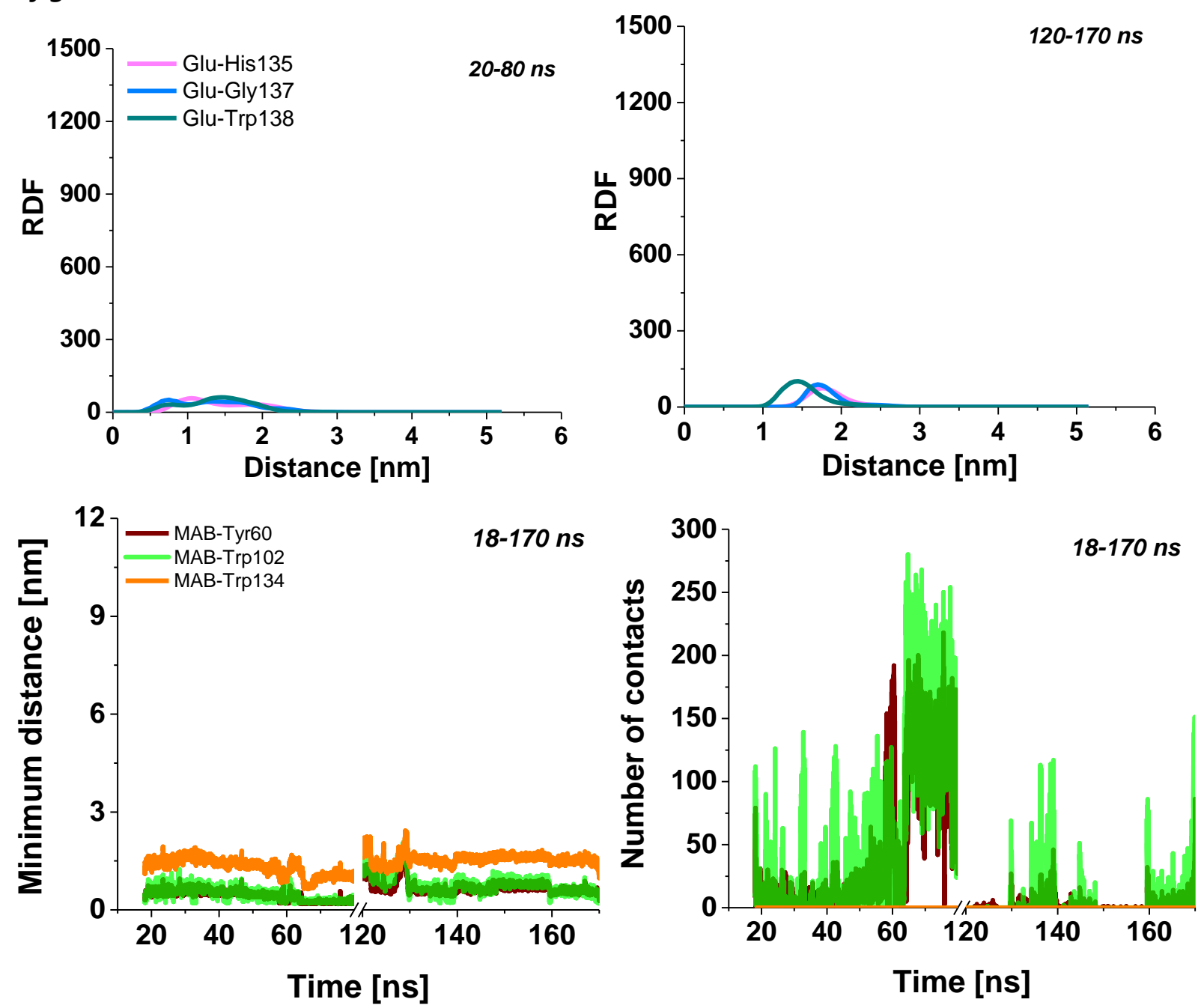

Figure S22: (top) Radial distribution functions of the distance between the glutamate part of MTX and the target interaction partners from FR $\alpha$, (middle) minimum distance and number of contacts between the methyl aminobenzoate (MAB) of MTX and the target interaction partners from FR $\alpha$, and (bottom) evolution of the number of hydrogen bonds between the glutamate of MTX and the target interaction partners from the binding pocket of FR $\alpha$ during the bound periods of Conf4 

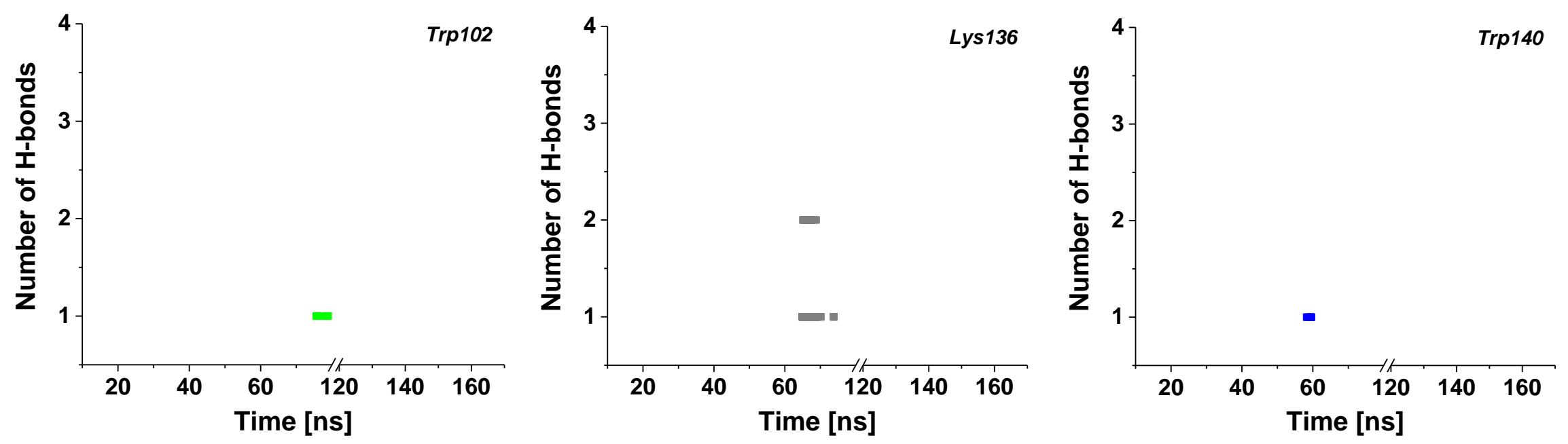

Figure S22: (continued)

The interactions of MTX with the target amino acids from the binding pocket of FR $\alpha$ (Figure S22) are much weaker than those of FA (Figures S14-S16). In the first bound period of Conf4, the glutamate part of the ligand has some proximity to Gly137 and Trp138 but both the position of the RDF maxima and their intensity do not signify strong coupling, compared to the folate. In the second bound period, the preferred distances are too large, which means that glutamate does not participate into stabilizing backbone interactions with the three target amino acids. The methyl aminobenzoate fragment of MTX is in close proximity to Tyr60 and Trp102 only in the first bound period, too. There, especially from 70 ns to 80 ns, massive hydrophobic coupling to these partners stabilizes the binding of the ligand. The hydrogen bonding of the glutamate of MTX is negligible in the first bound period and absent in the second one. This is due to the perpendicular positioning of this ligand at the pocket entrance and is in stark contrast to the behavior of FA in all its bound configurations. This loss of a combination of stabilizing factors is the reason for the inability of MTX to bind permanently to FR $\alpha$ in the same mode as folate.

The ligand adopts this inappropriate perpendicular alignment because of the strong propensity of methyl aminopterin to interact with Trp102 (Figure S23), one of the amino acids located at the binding site entrance. In both bound periods of Conf4, these two parts remain in close contact. 


\section{Configuration 4}
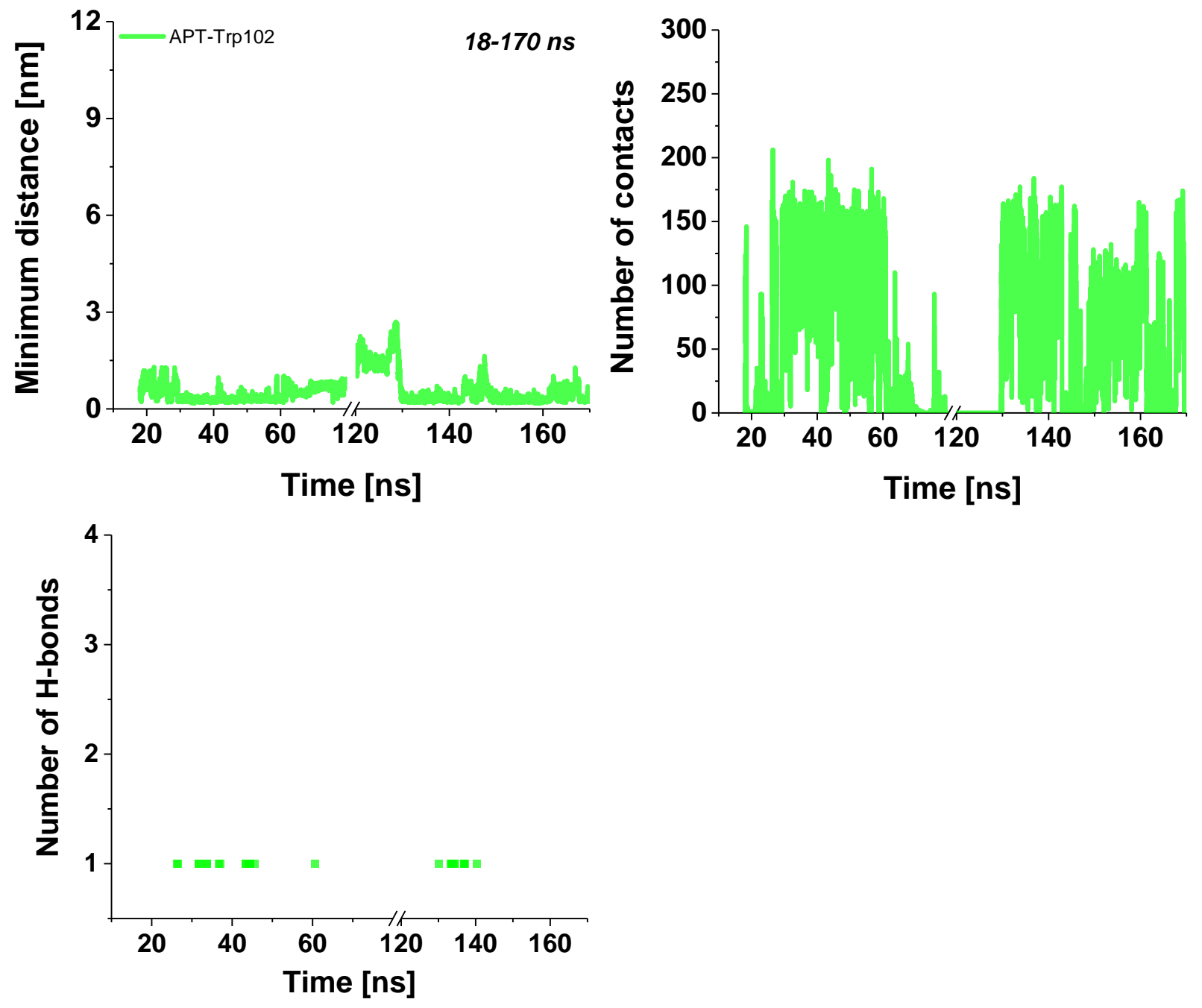

Figure S23: Evolution of (top) the minimum distance and number of contacts and (bottom) the number of hydrogen bonds between the aminopterin of MTX and Trp102 from FR $\alpha$ during the bound periods of Conf4 


\section{Raltitrexed}

The specific interactions of RTX in Conf1 and Conf3 (when it binds to the receptor active site) and in the other two trajectories are summarized in Figures S24 to S29 and Table S4. The glutamate part of the ligand $\pi$-stacks with its target amino acids from the pocket most of the time in Conf1 and Conf3. The only exceptions are the period 200-250 ns in Conf1 (Figures S24, S25) and 50-100 ns in Conf3 (Figure S24), where RTX is quite mobile. In Conf3, the binding of the molecule is stabilized additionally by coupling to Arg106. It is especially pronounced in the period 100-150 ns. There, the $\pi$-stacking to Arg106 and His135 is exceptionally strong (Figure S24). This might be related to the orientation rearrangement of RTX (see main text), which follows shortly afterwards. Apart from that, the $\pi$-stacking of glutamate is similar to that of FA in Conf1 and Conf3. An interesting feature of the binding of RTX is the non-negligible interaction of its $M Q$ fragment with its partner amino acids from the pocket of FR $\alpha$ (Figures S24, S25, and S27, Table S4). It is particularly remarkable in the period 150-200 ns of Conf3 and is a unique signature of the deep penetration of RTX into the cavity with its MQ part. Such interactions are not registered for any of the other ligands. The 'proper' binding of RTX is reflected also in the conduct of its MAT fragment. The latter is in close proximity to Tyr60 and Trp102 (similar to FA in Conf1 and MTX in Conf4) but in addition to that has sizeable contacts with Trp134 (Figures S25 and S26), which is not realized by the other two ligands.

The hydrogen bonding of glutamate is both qualitatively and quantitatively very similar to that of FA - H-bonds rapidly form and break but are most persistent with Lys136 and Trp140 (Table S4 and Figure S27) and very rare with Trp102. The binding of both ligands is assisted by Arg106 as well but mostly in Conf3. The rotation of MQ in Conf3 of RTX affects predominantly the hydrogen bonding with Trp140. H-bonds of MQ with His135, Arg103, and Asp81 are formed only in the last 40 ns of Conf3. This means that they become operative for stabilizing the ligand only when it is inserted very deeply into the pocket. The less pronounced degree of penetration of $\mathrm{MQ}$ in Conf1 is well illustrated by its very close proximity to Trp102 (situated at the pocket

entrance) and by its larger separation from Asp81 (located at the pocket bottom) (Figure S28). In contrast, MQ in Conf3 approaches constantly Asp81 and after the rotation at ca. 160 ns reaches distances $<1.0 \mathrm{~nm}$. This is the final evidence that RTX is completely inserted into the protein cavity in this trajectory.

As evident from its FR $\alpha$ interaction partners in Conf2 and Conf4 (Figure S29), they are similar in the two configurations and are located at the surface of the protein facing the lipid bilayer, opposite the binding pocket. The ligand is quite mobile but stays bound to the protein surface for long periods. As discussed already for MTX, electrostatic attraction seems to play a dominant role also for RTX to bind there because from 79 \% (second bound period of Conf2) to $96 \%$ (first bound period of Conf2) of the closest contacts of the ligand are with amino acids with charged side chains. However, the strong and persistent binding of RTX there (unlike MTX, which spends much less time) is likely to be additionally supported by spatial confinement between two loops of the protein structure and probably by other interactions, too. 

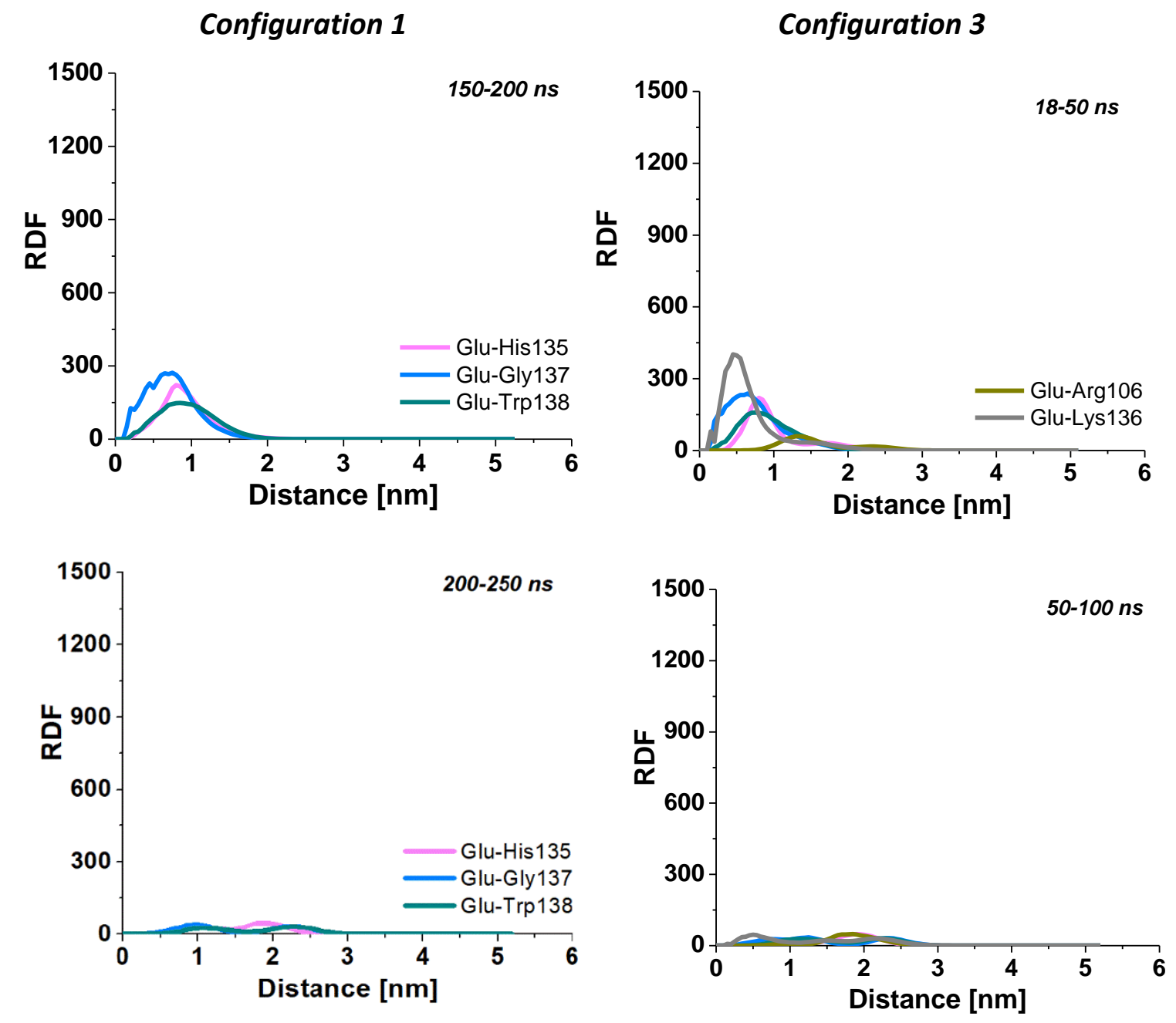

\section{Configuration 3}
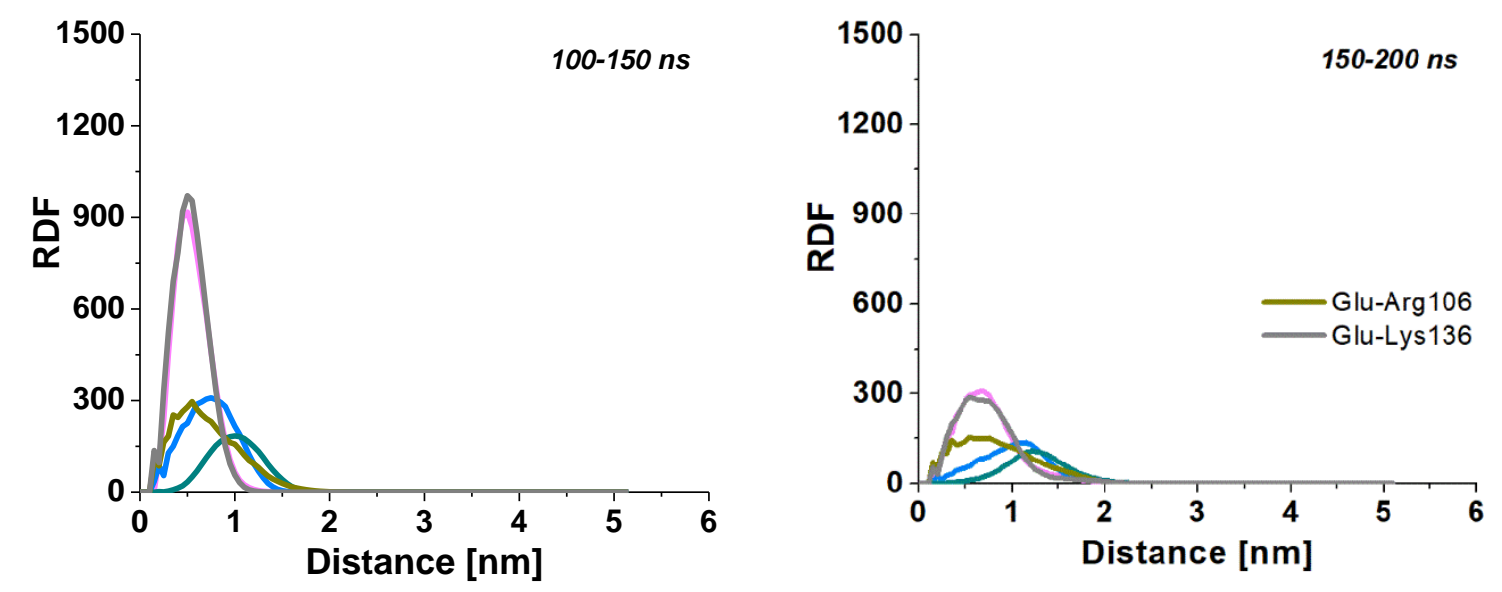

Figure S24: Radial distribution functions of the distance between the glutamate part of RTX and its target interaction partners from FR $\alpha$ during the bound periods of Conf1 and Conf3 


\section{Configuration 1}
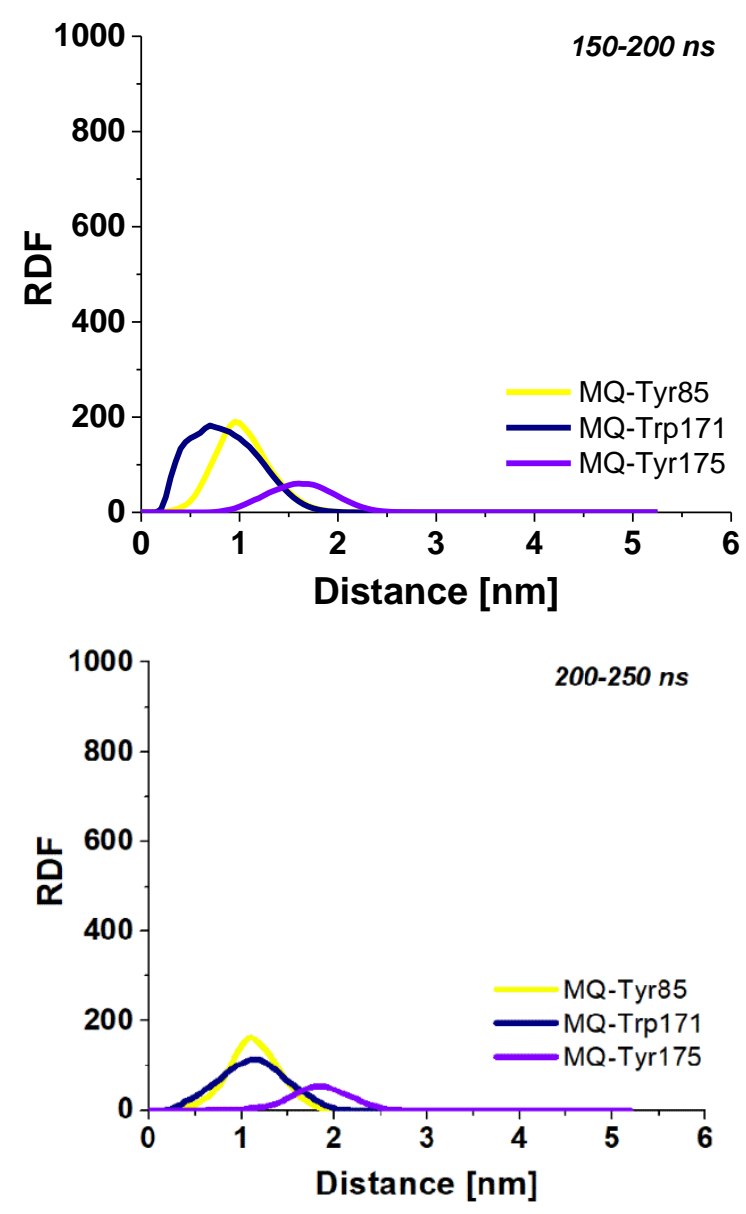

\section{Configuration 3}

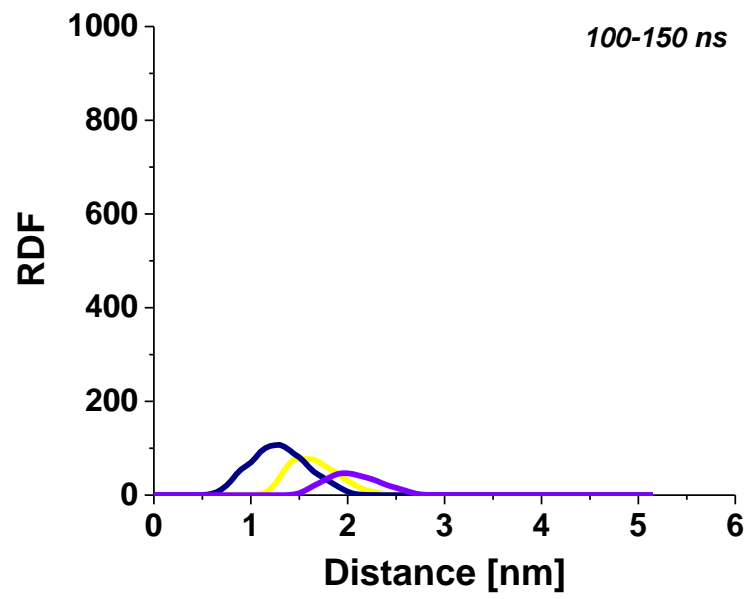

\section{Configuration 3}
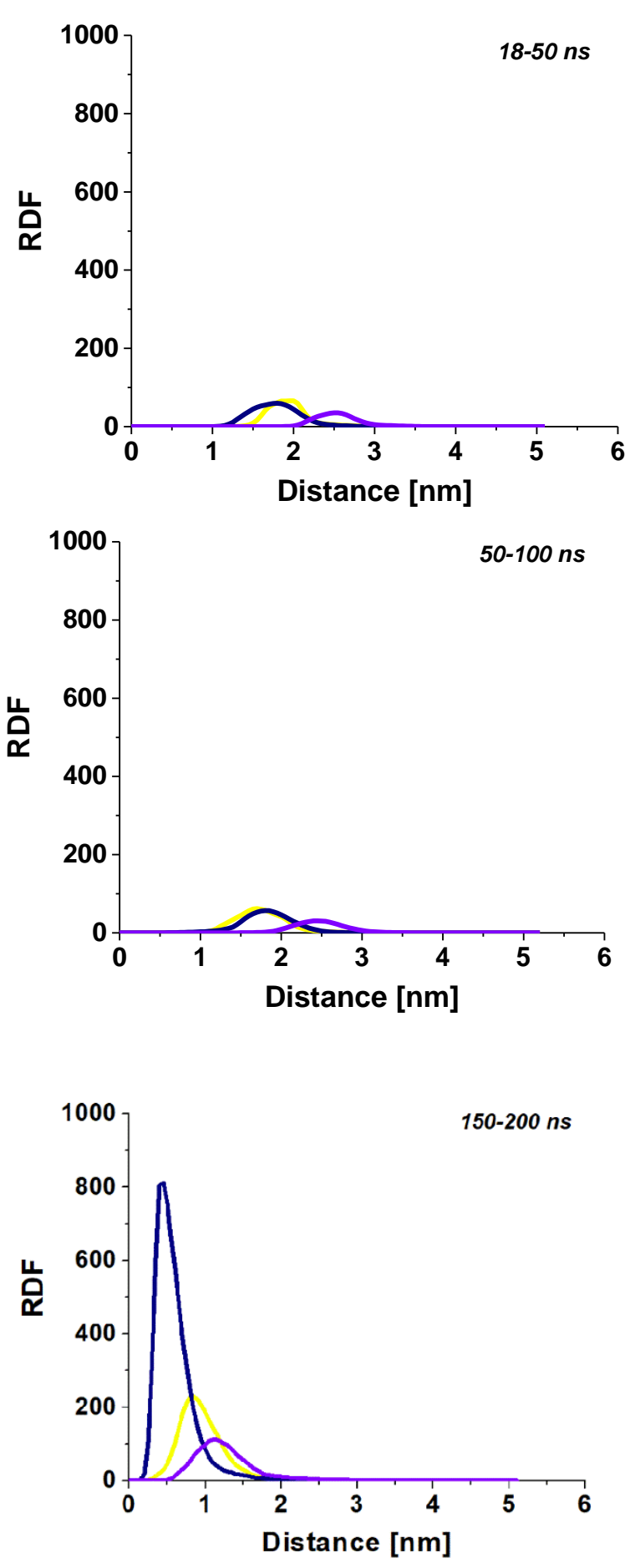

Figure S25: Radial distribution functions of the distance between the MQ fragment of RTX and its target interaction partners from FR $\alpha$ during the bound periods of Conf1 and Conf3 


\section{Configuration 1}

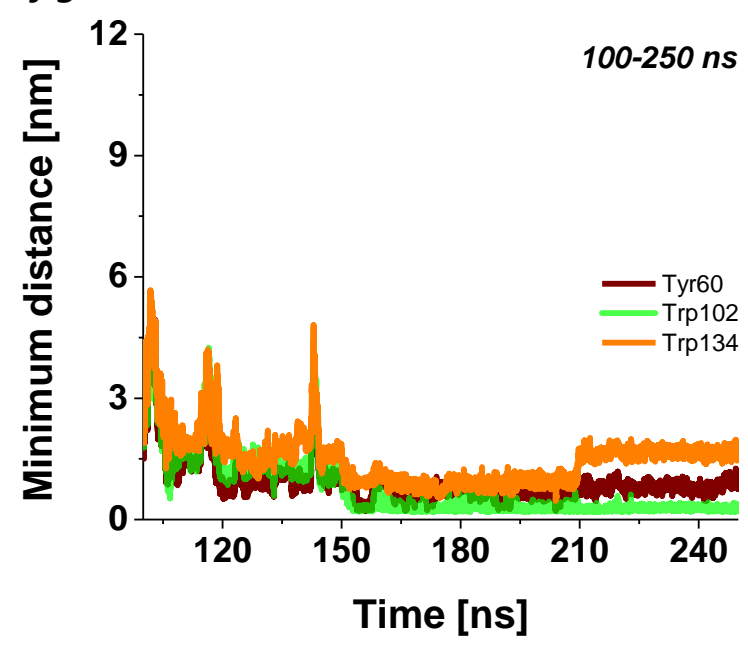

\section{Configuration 3}
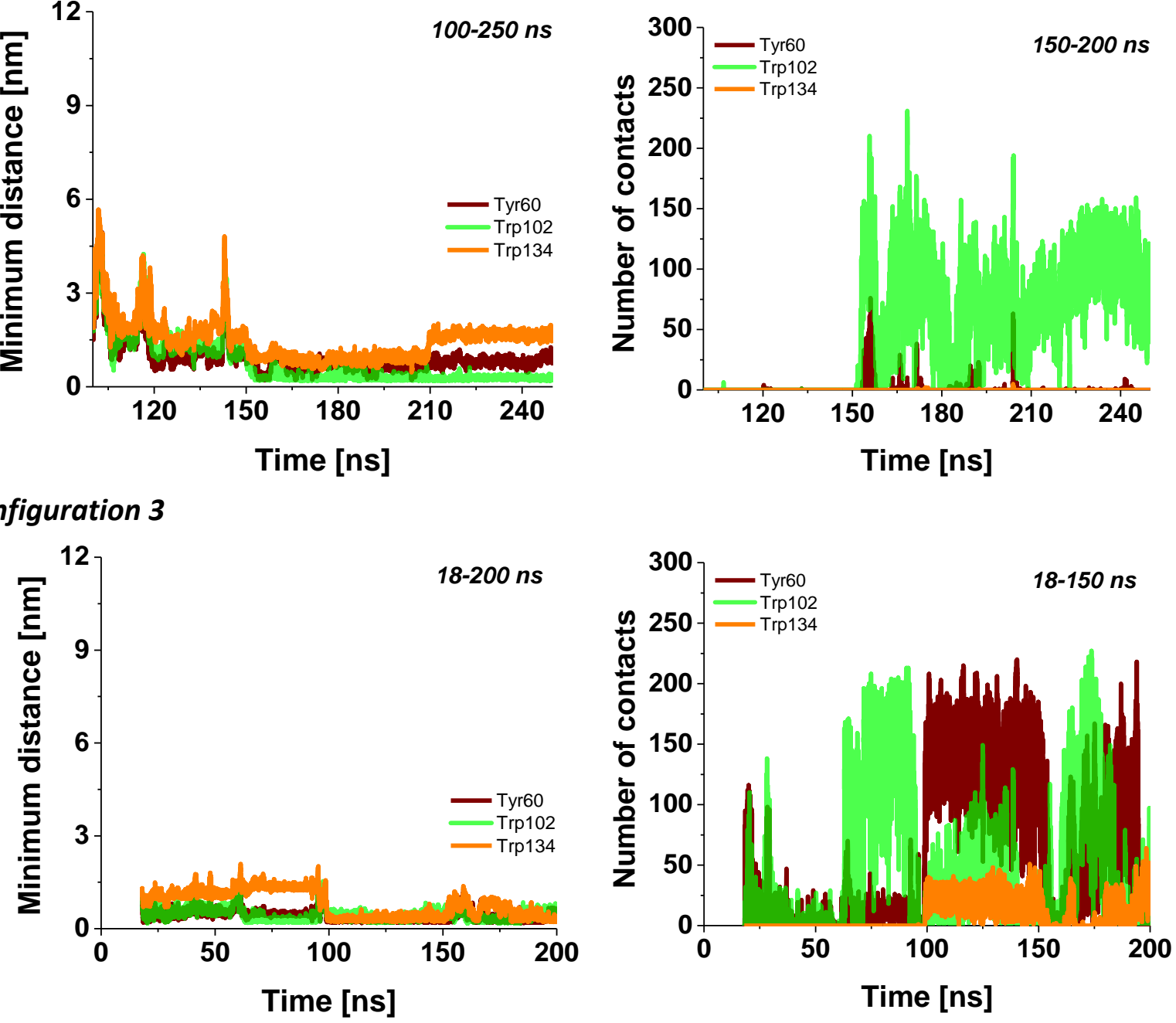

Figure S26: Evolution of the (left) minimum distance and (right) number of contacts during the entire bound period between atom pairs from the MAT fragment of RTX and its target interaction partners from FR $\alpha$ in Conf1 and Conf3 
Table S4: Average number $\left(\mathrm{N}_{H B}{ }^{\text {ave }}\right)$ with standard deviation, preferred length $\left(\mathrm{R}_{\mathrm{HB}}{ }^{\text {ave }}\right)$, and type of the hydrogen bonds formed between the glutamate of RTX and its target interaction partners from FR $\alpha$ during the bound periods of Conf1 and Conf3

\begin{tabular}{|c|c|c|c|c|}
\hline Amino acid & Period & $\mathbf{N}_{\mathrm{HB}}{ }^{\text {ave }}$ & $\mathrm{R}_{\mathrm{HB}}^{\text {ave }}, \mathrm{nm}$ & Bond type \\
\hline \multicolumn{5}{|c|}{ Configuration 1} \\
\hline \multirow[b]{2}{*}{ Trp102 } & $150-200 \mathrm{~ns}$ & $0.000 \pm 0.020$ & $0.218 / 0.233$ & \multirow{2}{*}{$\alpha$ - or $\gamma$-carboxylate group } \\
\hline & $200-250 \mathrm{~ns}$ & $0.005 \pm 0.075$ & 0.213 & \\
\hline \multirow{2}{*}{ Lys136 } & $150-200 \mathrm{~ns}$ & $0.266 \pm 0.459$ & 0.168 & \multirow{2}{*}{ both carboxylate groups } \\
\hline & $200-250 \mathrm{~ns}$ & $0.062 \pm 0.258$ & 0.173 & \\
\hline \multirow{2}{*}{ Trp140 } & $150-200 \mathrm{~ns}$ & $0.116 \pm 0.322$ & 0.188 & \multirow[b]{2}{*}{ both carboxylate groups } \\
\hline & $200-250 \mathrm{~ns}$ & $0.003 \pm 0.055$ & $\begin{array}{c}0.193 \text { to } \\
0.223\end{array}$ & \\
\hline \multicolumn{5}{|c|}{ Configuration 3} \\
\hline \multirow{4}{*}{ Trp102 } & $18-50 \mathrm{~ns}$ & $0.000 \pm 0.018$ & 0.243 & \multirow{4}{*}{$\alpha$-carboxylate group } \\
\hline & $50-100 \mathrm{~ns}$ & $0.000 \pm 0.000$ & ----- & \\
\hline & $100-150 \mathrm{~ns}$ & $0.000 \pm 0.000$ & $\begin{array}{ll}---- \\
\end{array}$ & \\
\hline & $150-200 \mathrm{~ns}$ & $0.000 \pm 0.024$ & $\begin{array}{c}0.188 / 0.213 \\
/ 0.223\end{array}$ & \\
\hline \multirow{4}{*}{ Lys136 } & $18-50 \mathrm{~ns}$ & $0.319 \pm 0.563$ & 0.163 & \multirow{4}{*}{ both carboxylate groups } \\
\hline & $50-100 \mathrm{~ns}$ & $0.037 \pm 0.199$ & 0.173 & \\
\hline & $100-150$ ns & $0.572 \pm 0.555$ & 0.168 & \\
\hline & $150-200 \mathrm{~ns}$ & $0.235 \pm 0.440$ & 0.168 & \\
\hline \multirow{4}{*}{ Trp140 } & $18-50 \mathrm{~ns}$ & $0.161 \pm 0.371$ & 0.188 & \multirow{4}{*}{ both carboxylate groups } \\
\hline & $50-100 \mathrm{~ns}$ & $0.015 \pm 0.125$ & 0.203 & \\
\hline & $100-150 \mathrm{~ns}$ & $0.005 \pm 0.073$ & 0.188 & \\
\hline & $150-200 \mathrm{~ns}$ & $0.008 \pm 0.089$ & 0.195 & \\
\hline \multirow{4}{*}{ Arg106 } & $18-50 \mathrm{~ns}$ & $0.000 \pm 0.000$ & ---- & \multirow{4}{*}{ 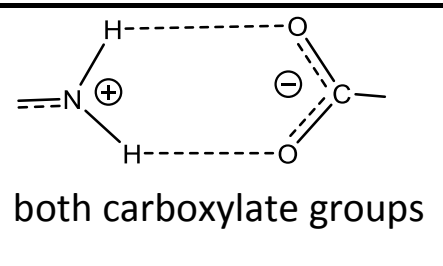 } \\
\hline & $50-100 \mathrm{~ns}$ & $0.000 \pm 0.000$ & $\begin{array}{ll}---- \\
\end{array}$ & \\
\hline & $100-150 \mathrm{~ns}$ & $0.754 \pm 0.850$ & 0.170 & \\
\hline & $150-200 \mathrm{~ns}$ & $0.490 \pm 0.807$ & 0.173 & \\
\hline
\end{tabular}




\section{Configuration 1}
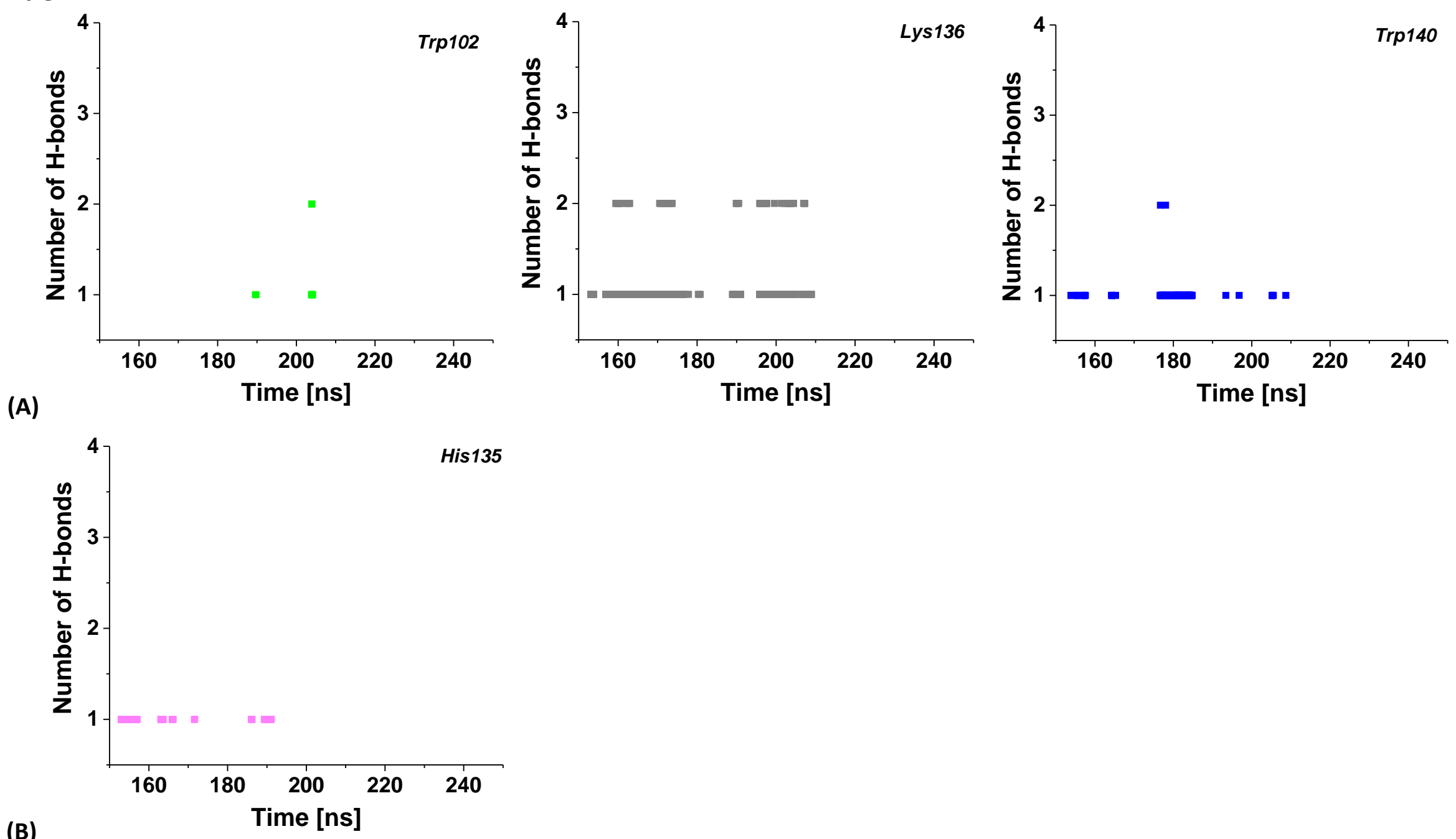

Figure S27: Evolution of the number of hydrogen bonds between (A) the glutamate or (B) the MQ of RTX and their target interaction partners from the binding pocket of FR $\alpha$ during the bound periods of Conf1 and Conf3; no hydrogen bonds were formed between MQ and Arg103, Arg106, Asp81, and Ser174 in Conf1 


\section{Configuration 3}
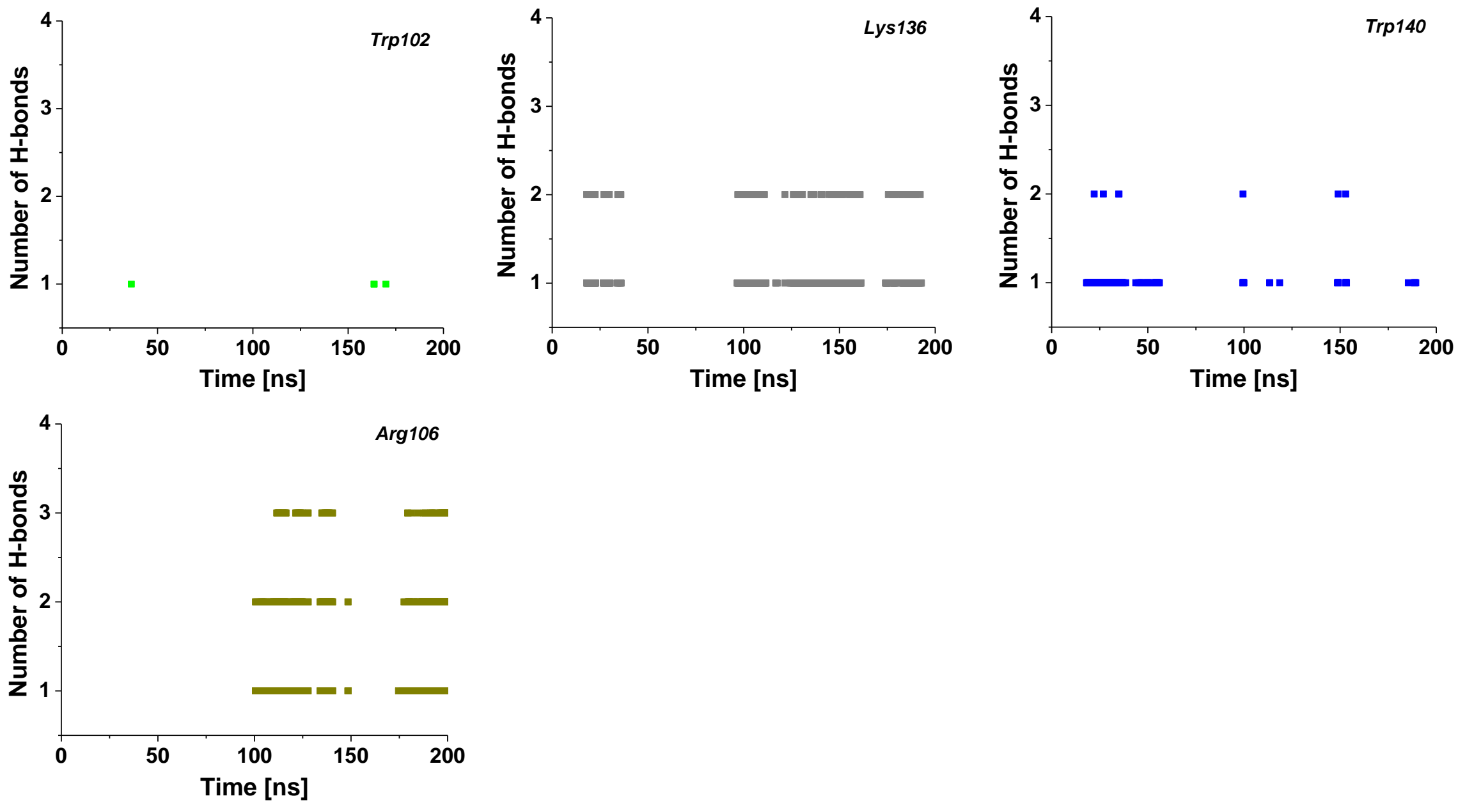

(A)

Figure S27: (Continued) 

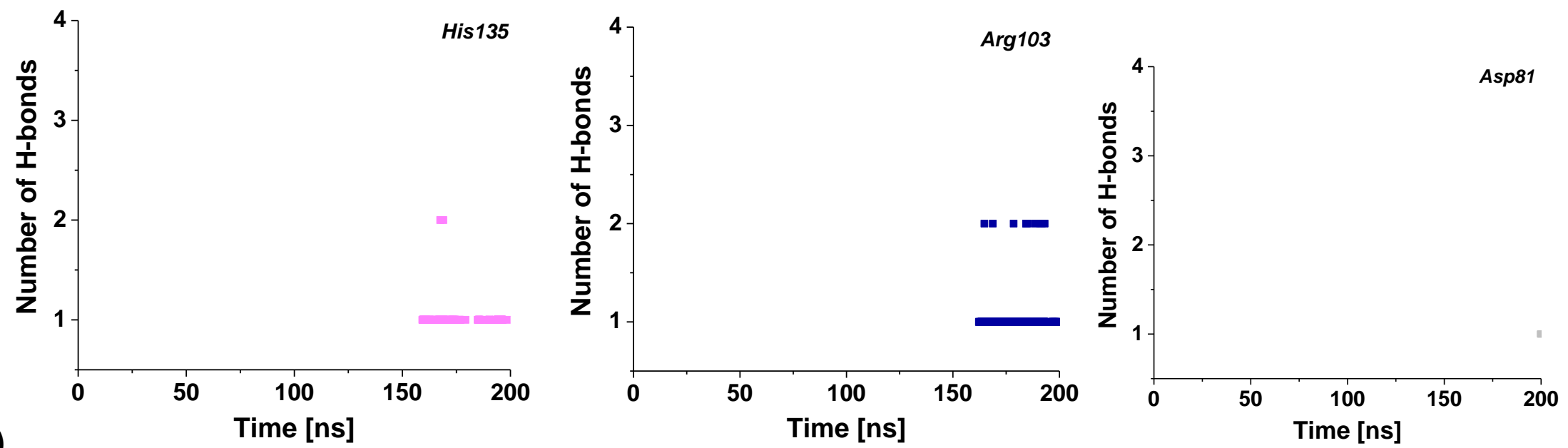

Figure S27: (Continued) 


\section{Configuration 1}

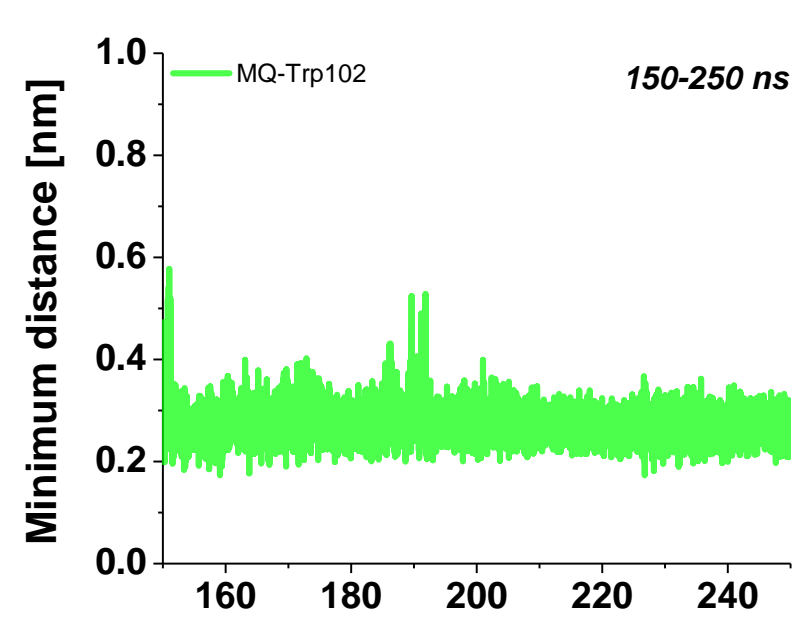

(A)

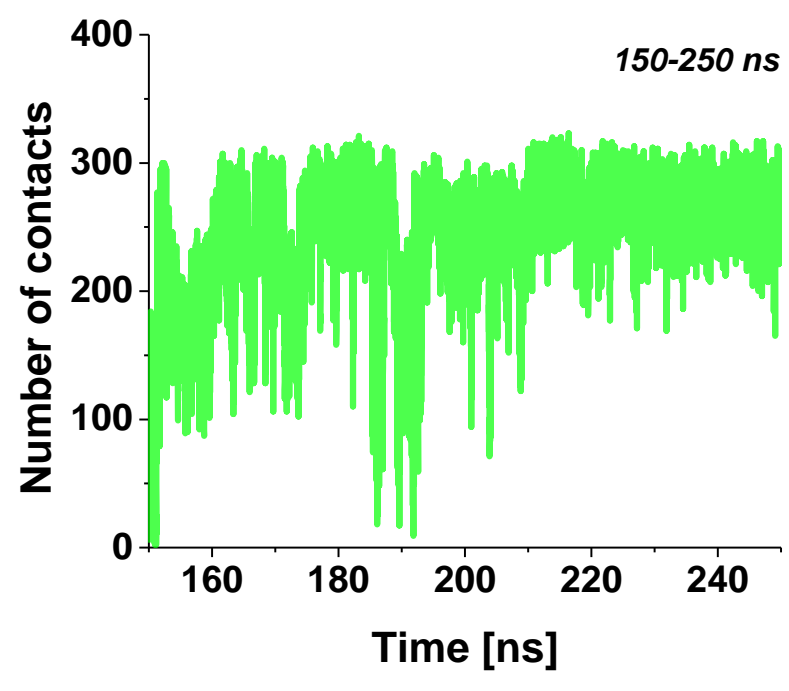

Configuration 1

Configuration 3
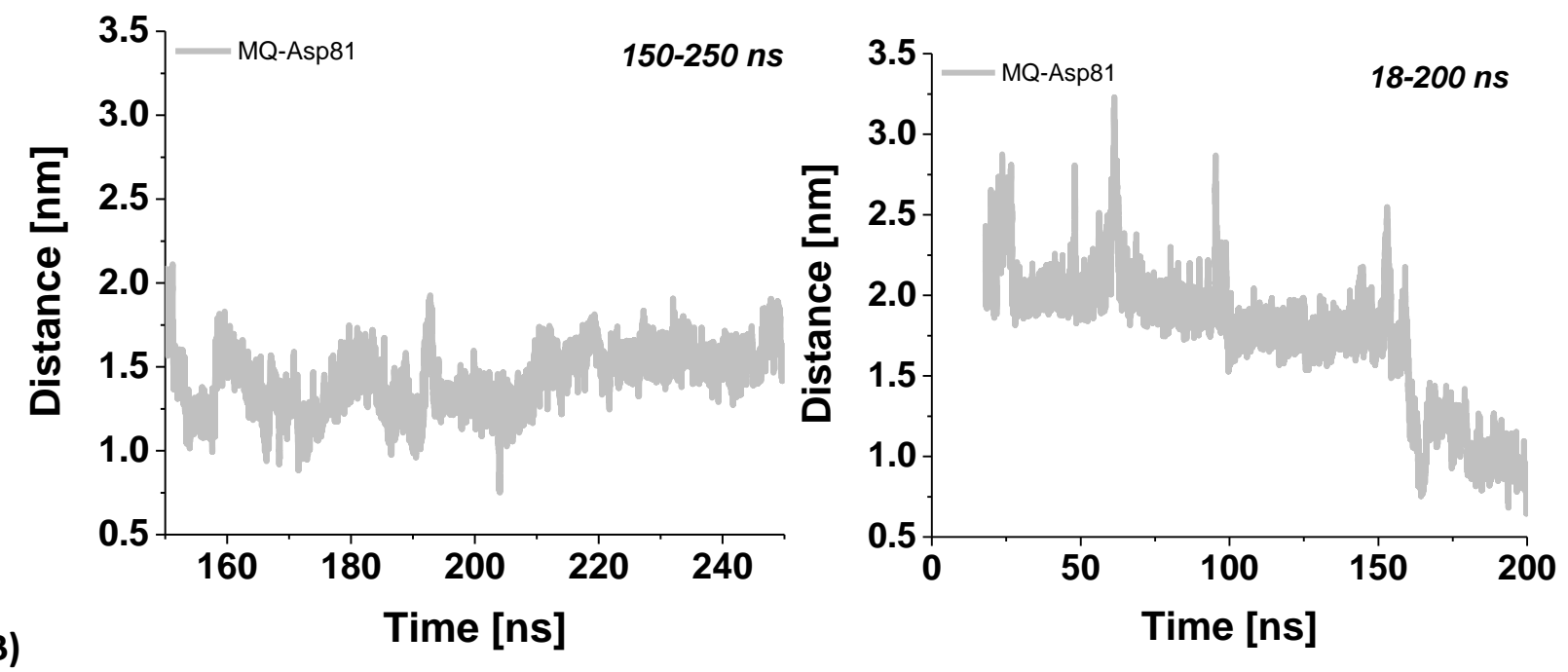

Figure S28: (A) Evolution of the (left) minimum distance and (right) number of contacts between atom pairs from the MQ fragment of RTX and Trp102 from FR $\alpha$ during the entire bound period of Conf1; (B) distance between the COMs of MQ from RTX and Asp81 from FR $\alpha$ during the entire bound time of Conf1 and Conf3 

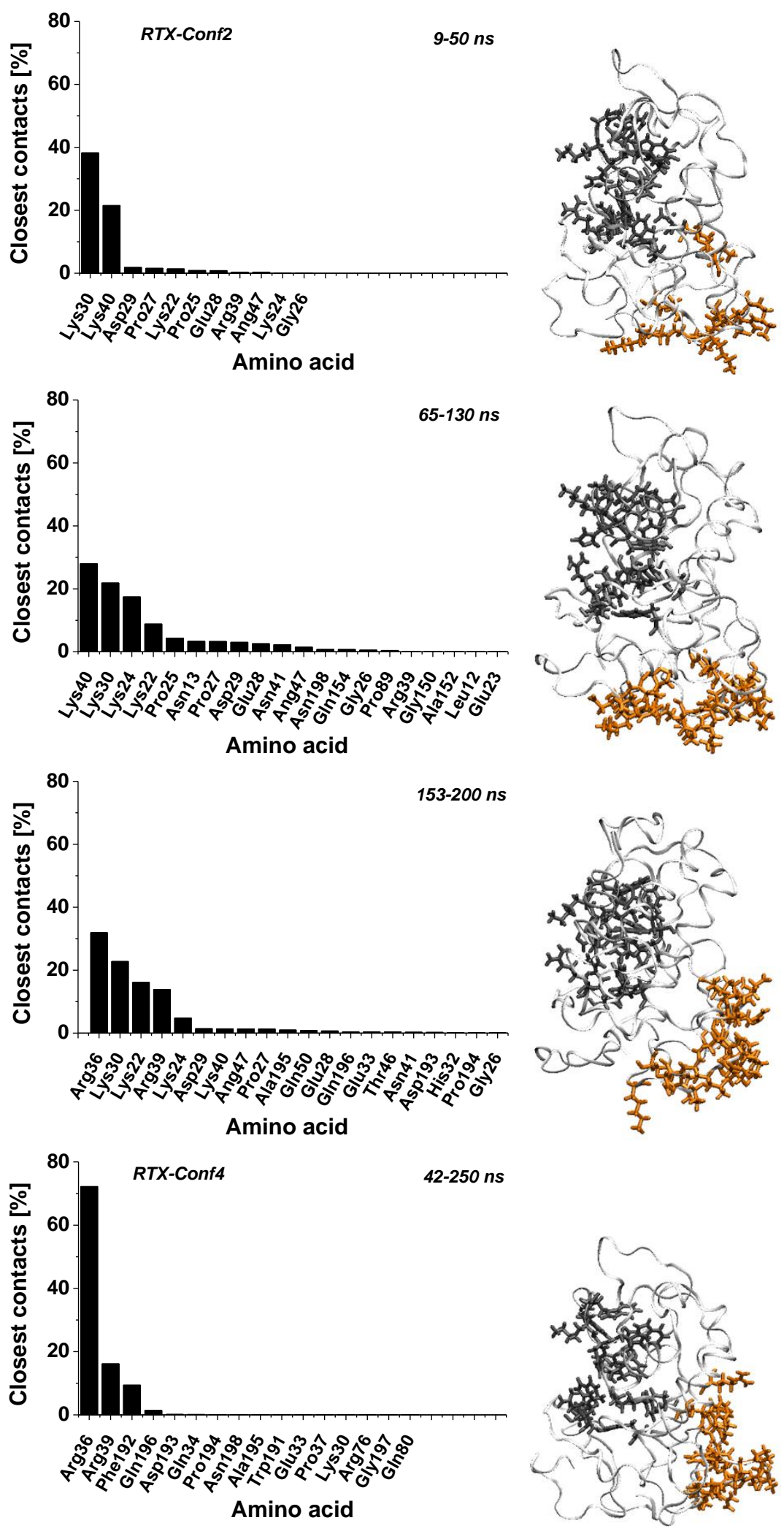

Figure S29: (left) Distribution of the number of minimum distances closed between RTX and amino acids from FR $\alpha$ during the bound periods of Conf2 (top) and Conf4 (bottom); (right) illustration of these interaction partners on the structure of the receptor on the last frame of the particular period; the protein is shown as ribbons, the binding pocket residues - as grey licorice, the interaction partners of RTX - as orange licorice 


\section{Pemetrexed}

The analyses of the specific interactions of PTX are collected in Figures S30 to S33 and Table S5. The different pattern of interaction of the glutamate of PTX with the pocket residues, compared to that of FA and RTX, is evident from the RDFs in Figure S30. Glu of PTX interacts very intensely with Gly137 and Trp138 during the first 100 ns of the bound periods of Conf1, while His135 and Gly135 are the more preferred RDF neighbors of FA and RTX. The peaks both in Conf1 in the next $150 \mathrm{~ns}$ and in the entire bound period of Conf2 are very weak and shallow and centered at distances larger than $0.5 \mathrm{~nm}$. This is due to the retreat of the ligand from the back of the binding site of FR $\alpha$ discussed in the main text. The hydrogen bonding of the glutamate of PTX (Figure S31 and Table S5) is also not that pronounced as that of folate (Figure S16 and Table S3) and raltitrexed (Figure S27 and Table S4). PTX is stabilized by H-bonds only in Conf1. There, it interacts only with Lys136 during the complete bound period. Some hydrogen bonding also takes place with Trp140 but it breaks after the rearrangement of PTX at ca. 113 ns. Trp102 does not Hbond with glutamate. In Conf2 of PTX, Glu even does not form H-bonds with Lys136 and Trp140. This verifies that PTX is not stabilized by the same set of specific interactions as FA and RTX, when bound to FR $\alpha$.

The analysis of the amino acids from FR $\alpha$, which are closest to PTX during all bound periods (Figure S32), reveals the most specific feature of the behavior of this vector ligand. It can be seen from the histograms that PTX has different preferred partners from the protein in the four trajectories. They also change over time within a given trajectory.

This is in support of the ability of PTX to adsorb on the protein surface in a non-specific manner, which is discussed in the main text. The latter is in stark contrast to the conduct of RTX, which has almost identical preferred partners in Conf2 and Conf4 (Figure S29). PTX in Conf3 and Conf4 adsorbs at the same allosteric spot at the base of FR $\alpha$ but is able to change its interaction partners much more than RTX. Moreover, the specific interactions of RTX at the allosteric site are almost exclusively electrostatic (see main text), while those of PTX are of different nature. In most of the trajectories (with the exception of Conf4 after 50 ns where the locking of PTX between the two loops was stabilized $>80 \%$ electrostatically in a way very similar to RTX in Conf4) only between $20 \%$ and $50 \%$ of the closest amino acids of pemetrexed have charged side chains. This implies that the strong adsorption of PTX on the surface of the receptor is driven by other types of interactions, such as van der Waals attraction or hydrogen bonding. The former assumption is confirmed by the persistent large number of contacts between PTX and FR (Figure S4). The hydrogen bonding is checked by calculating the number of $\mathrm{H}$-bonds between the relevant fragments of PTX (ethyl benzoate (EB) in Conf2 and pyrollo[2,3-d]pyrimidinyl (PP) in the other three trajectories) and their closest partners from FR $\alpha$ (Figure S33). The intermolecular stabilization by $\mathrm{H}$-bonding is negligible in Conf1 and Conf2 and somewhat more important in Conf3 and Conf4. However, even in the latter two trajectories it does not look deterministic for the binding of PTX. 
Configuration 1
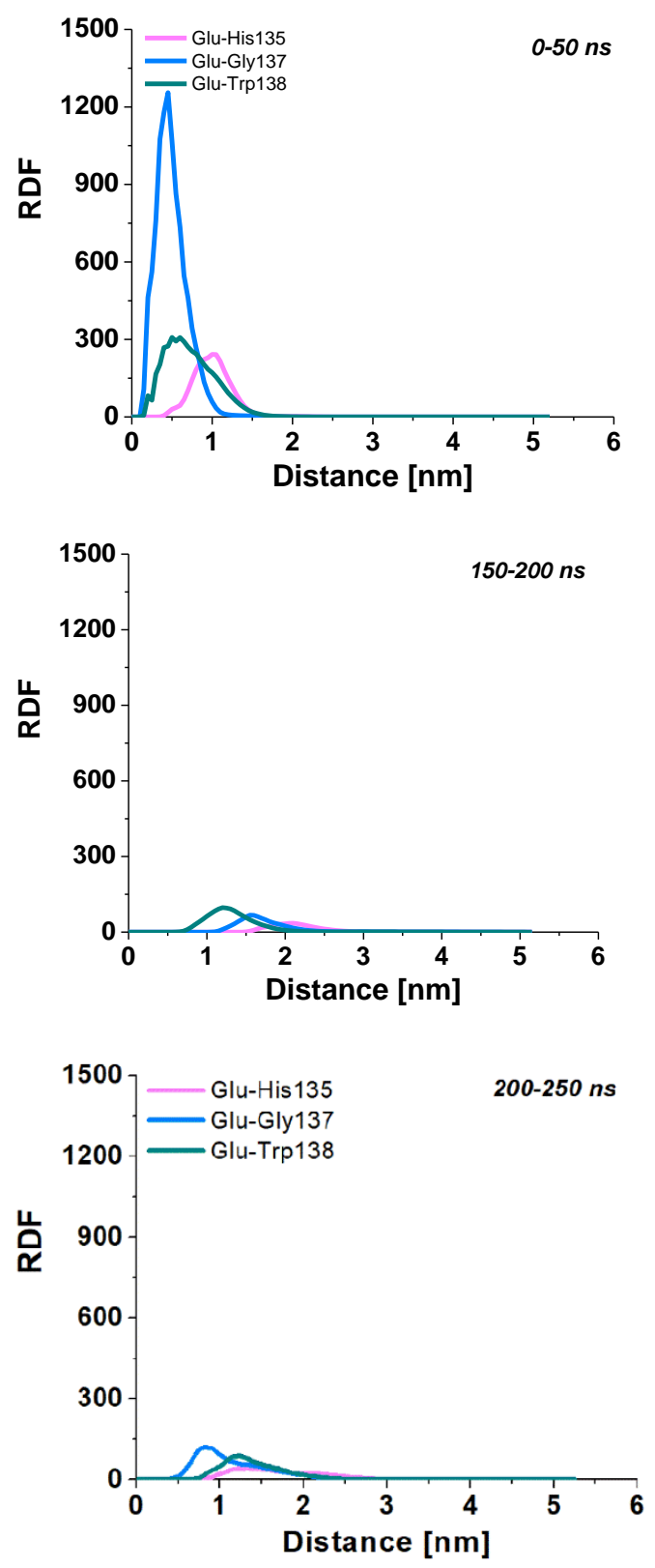

Configuration 2

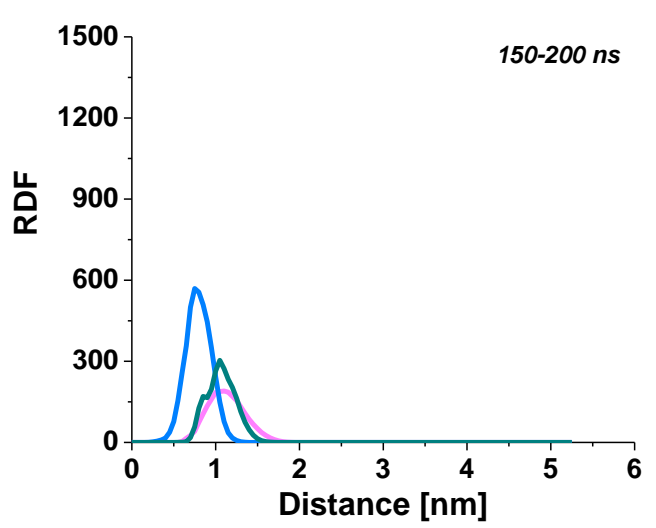

Configuration 1
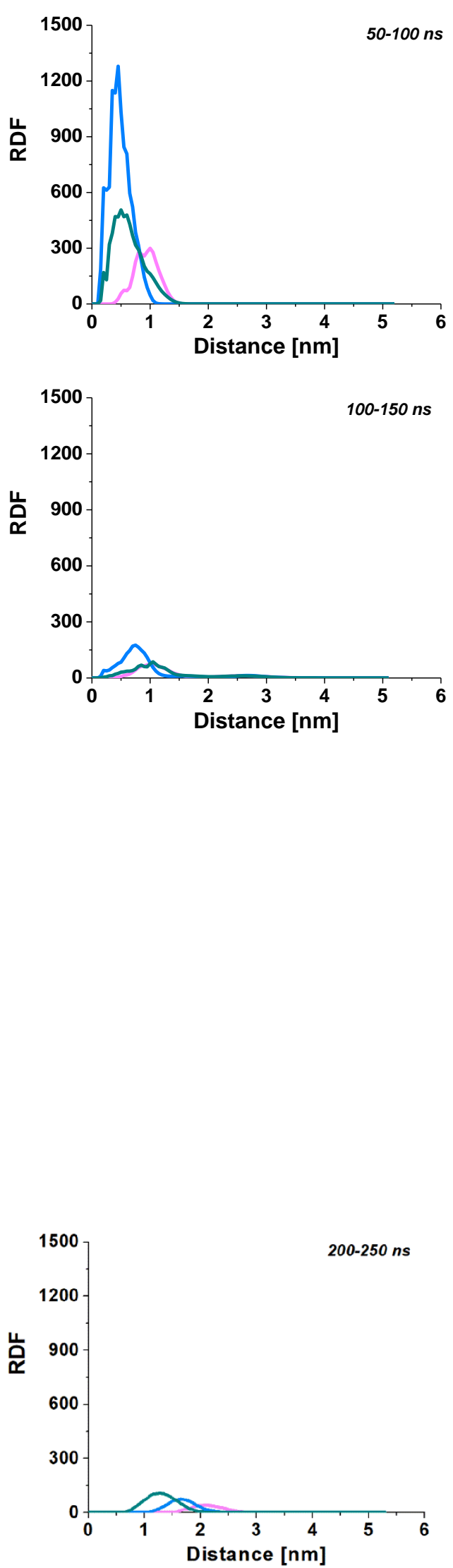

Figure S30: Radial distribution functions of the distance between the glutamate part of PTX and its target interaction partners from FR $\alpha$ during the bound periods of Conf1 and Conf2 
Table S5: Average number $\left(\mathrm{N}_{\mathrm{HB}}{ }^{\text {ave }}\right)$ with standard deviation, preferred length $\left(\mathrm{R}_{\mathrm{HB}}{ }^{\text {ave }}\right)$, and type of the hydrogen bonds formed between the glutamate of PTX and its target interaction partners from FR $\alpha$ during the bound periods of Conf1; no hydrogen bonds were formed with these amino acids in the other three trajectories and with Trp102 in any trajectory

\begin{tabular}{|c|c|c|c|c|}
\hline Amino acid & Period & $\mathbf{N}_{\mathrm{HB}}{ }^{\text {ave }}$ & $\mathrm{R}_{\mathrm{HB}}^{\text {ave }}, \mathrm{nm}$ & Bond type \\
\hline \multicolumn{5}{|c|}{ Configuration 1} \\
\hline \multirow{5}{*}{ Lys136 } & $0-50 \mathrm{~ns}$ & $0.276 \pm 0.466$ & 0.163 & \multirow{5}{*}{ both carboxylate groups until } \\
\hline & $50-100 \mathrm{~ns}$ & $0.245 \pm 0.506$ & 0.168 & \\
\hline & $100-150 \mathrm{~ns}$ & $0.065 \pm 0.260$ & 0.173 & \\
\hline & $150-200 \mathrm{~ns}$ & $0.093 \pm 0.297$ & 0.168 & \\
\hline & $200-250 \mathrm{~ns}$ & $0.004 \pm 0.060$ & 0.158 & \\
\hline \multirow{5}{*}{ Trp140 } & $0-50 \mathrm{~ns}$ & $0.154 \pm 0.368$ & 0.193 & \multirow{5}{*}{$\begin{array}{r}\text { only } \gamma-\mathrm{COO}^{-} ; \text {both carboxylate } \\
\text { groups }\end{array}$} \\
\hline & $50-100 \mathrm{~ns}$ & $0.224 \pm 0.424$ & 0.188 & \\
\hline & $100-150 \mathrm{~ns}$ & $0.051 \pm 0.220$ & 0.193 & \\
\hline & $150-200 \mathrm{~ns}$ & $0.000 \pm 0.000$ & ----- & \\
\hline & $200-250 \mathrm{~ns}$ & $0.000 \pm 0.000$ & ----- & \\
\hline
\end{tabular}




\section{Configuration 1}
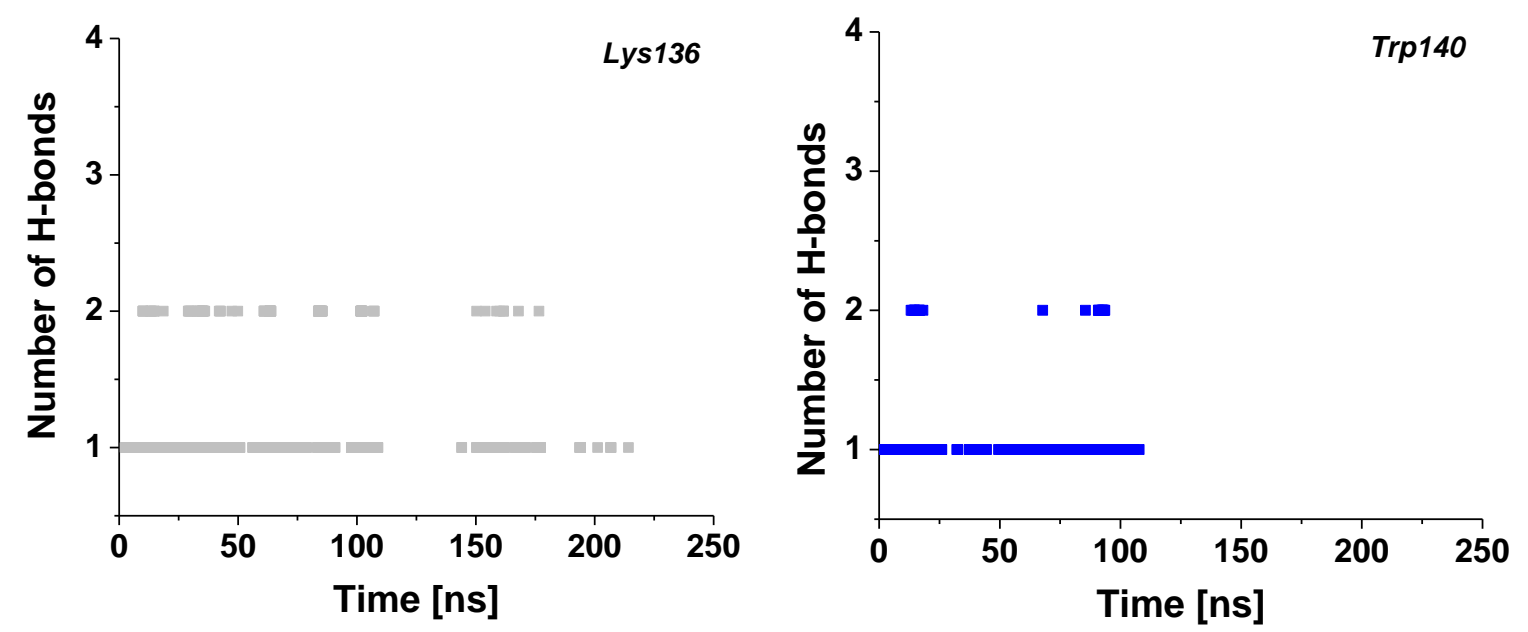

Figure S31: Evolution of the number of hydrogen bonds between the glutamate of PTX and its target interaction partners from the binding pocket of FR $\alpha$ during the bound periods of Conf1; no hydrogen bonds were formed with Trp102 in any of the configurations and with Lys136 and Trp140 in the other three trajectories 

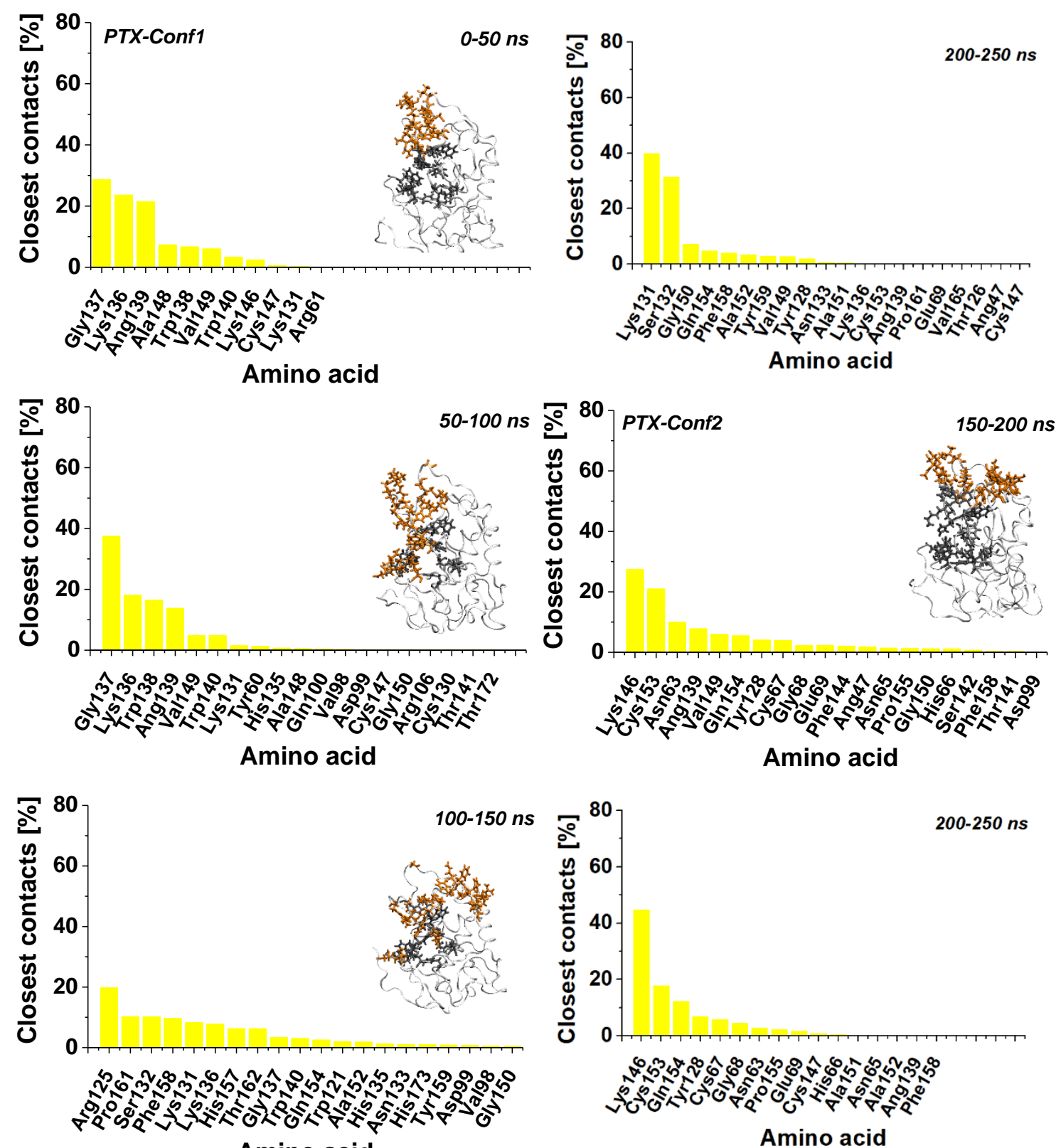

Amino acid

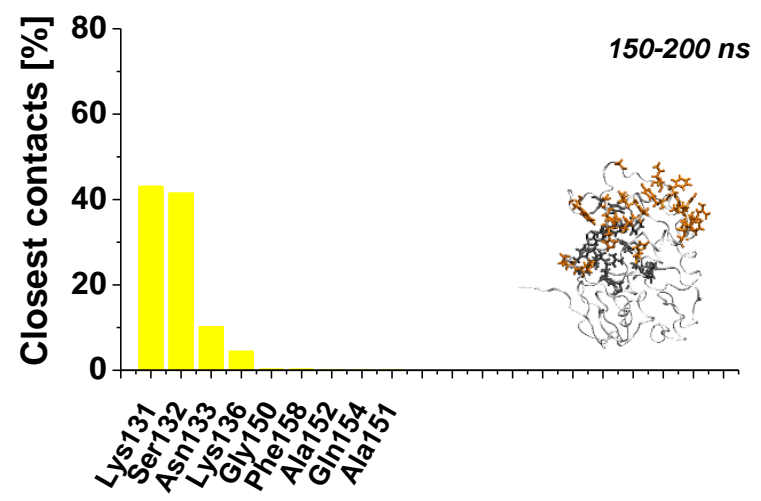

Amino acid

Figure S32: Distribution of the number of minimum distances closed between PTX and amino acids from FR $\alpha$ during the bound periods of (left) Conf1 (top) and Conf3 (bottom) and (right) Conf2 (top) and Conf4 (bottom); illustration of these interaction partners on the structure of the receptor on the last frame of the particular period is given as inset, where the interaction partners of PTX are depicted in orange licorice 


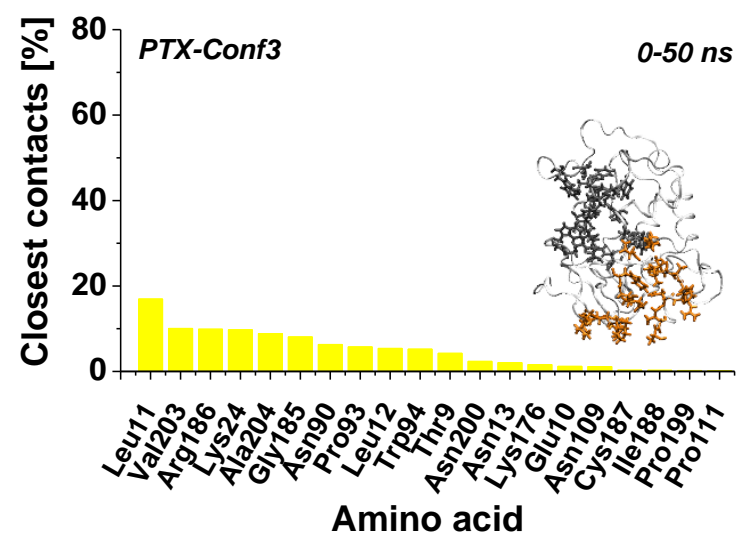

\section{Configuration 4}
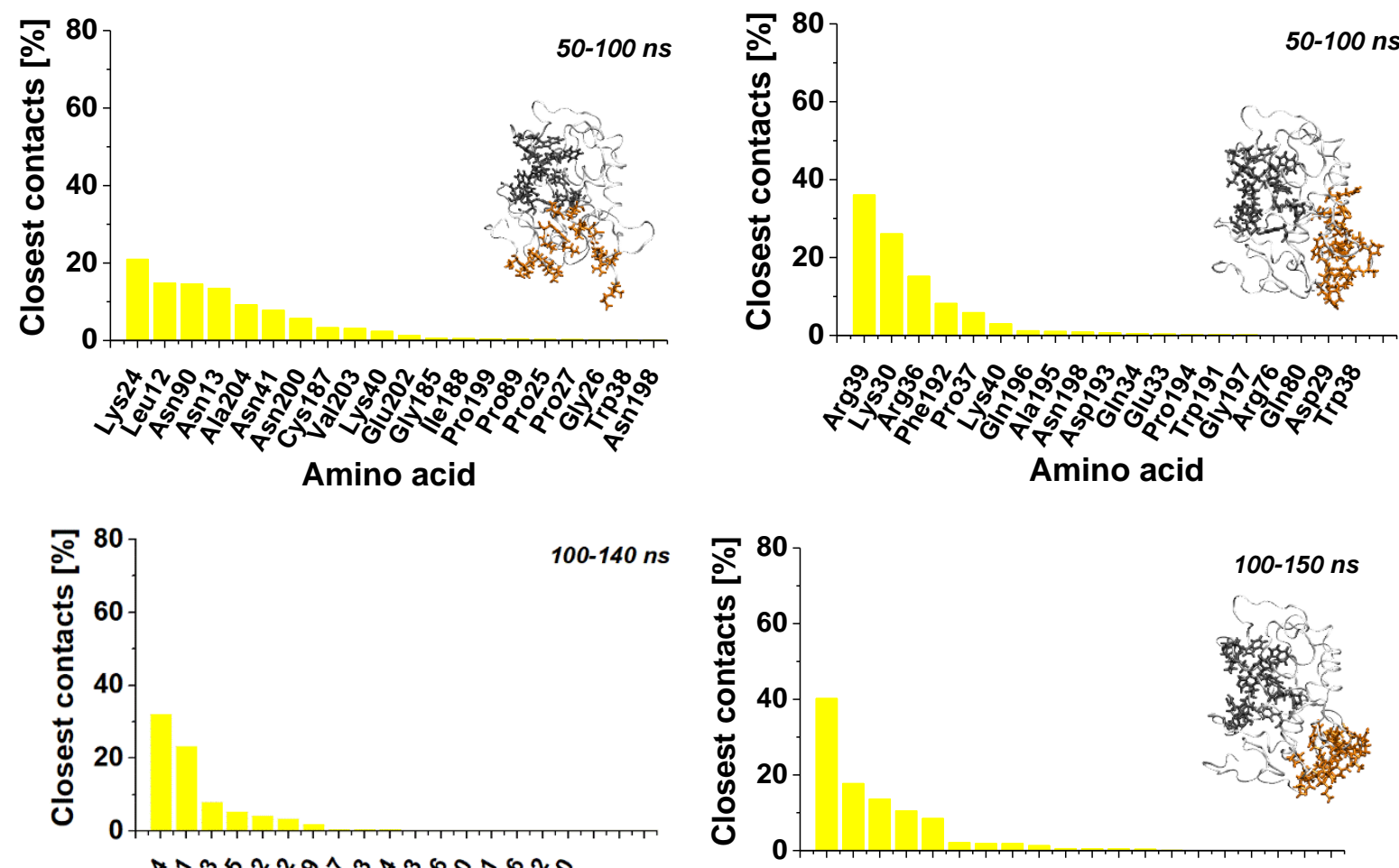

Amino acid

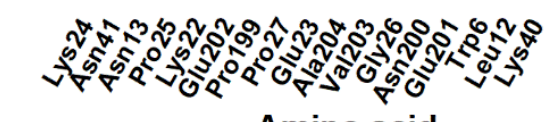

Amino acid

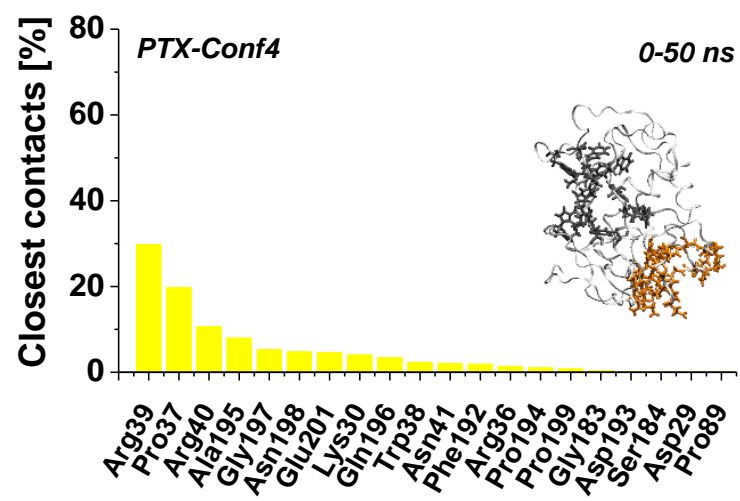

Amino acid

Figure S32: (Continued)

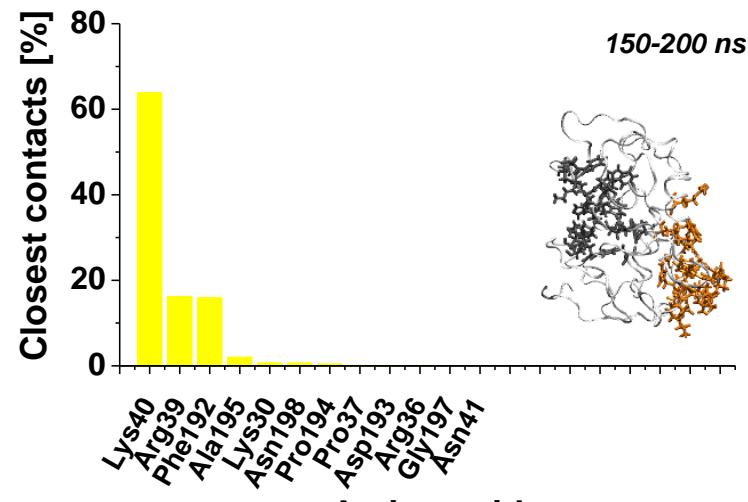

Amino acid 


\section{Configuration 1}

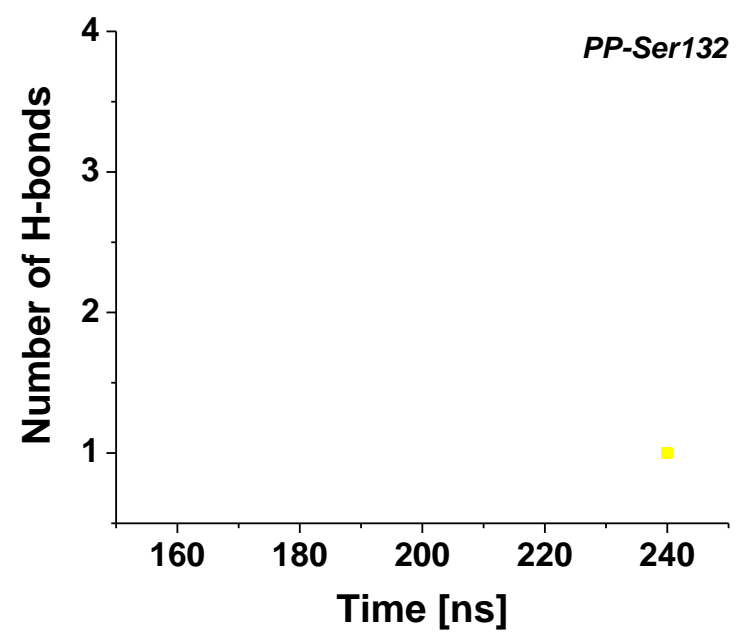

\section{Configuration 3}

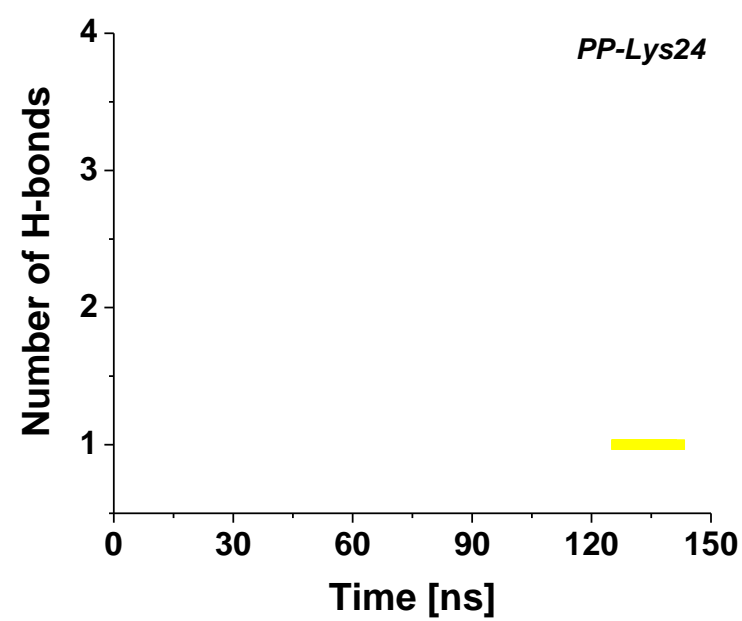

\section{Configuration 4}

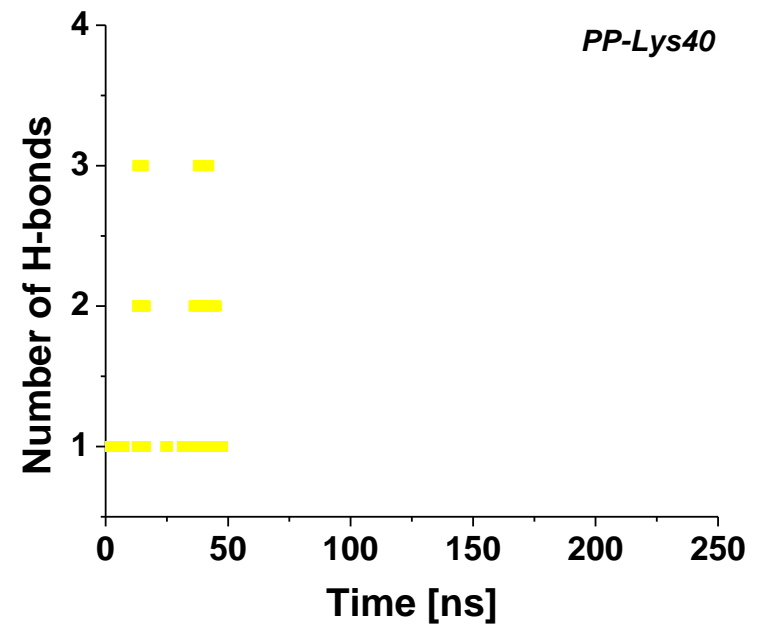

Configuration 2
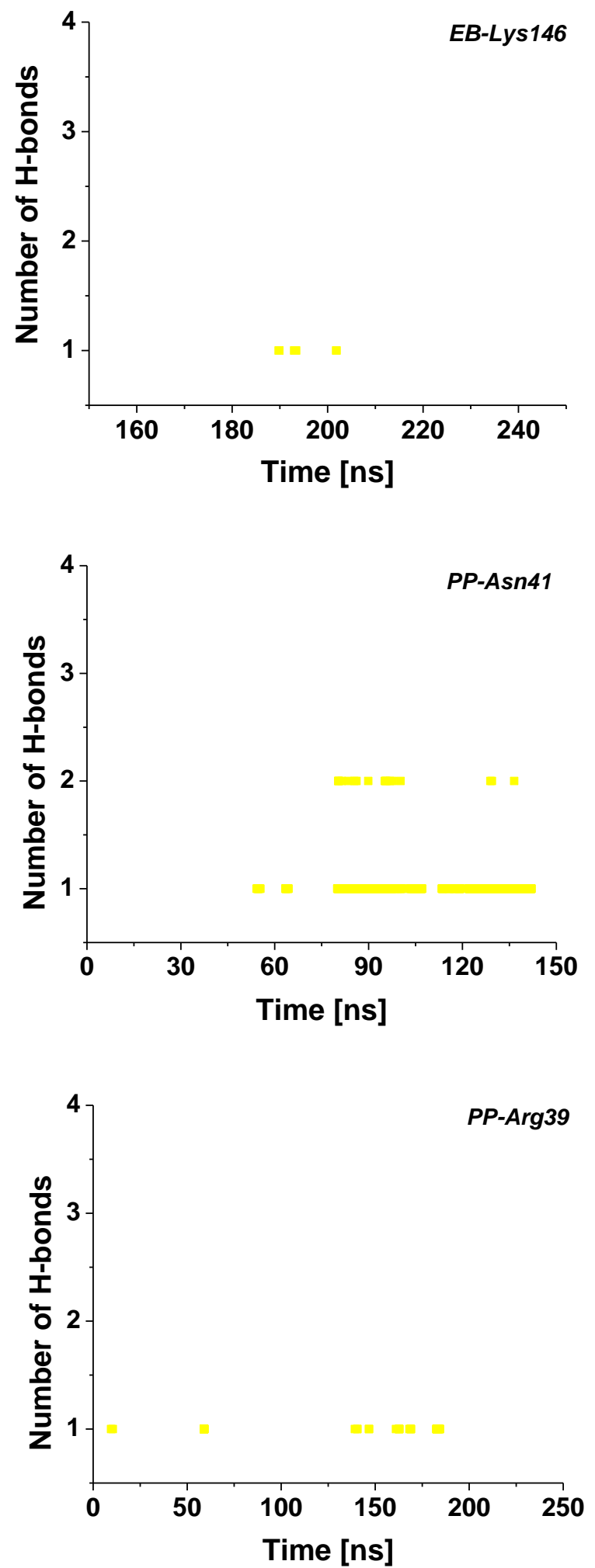

Figure S33: Evolution of the number of hydrogen bonds between the most intensively interacting fragment of PTX and its closest amino acids from FR $\alpha$ during the closely bound periods of Conf1 to Conf4 


\section{5-methyltetrahydrofolate}

The specific interactions of MTHF are presented in Table S6 and Figures S34 to S40. The data show that MTHF, compared to FA, preserves the specificity of binding to the active site but only to some extent. This ligand has measurable interactions with the target amino acids from the pocket (Figures S34 to S36) in three (Conf2, Conf3, and Conf4) out of the four trajectories (FA has in all of them). The similar orientation of MTHF at the pocket entrance to that of FA is especially evident in Conf2 where Gly137 is the preferred partner for backbone interactions of glutamate and aminobenzoate couples comparably to Tyr60 and Trp102 (the picture is alike for FA in Conf1, Figure S14, top). It should also be noted that tetrahydropterine (THP) does not bind to the target amino acids (the same as pterin of FA).

There are, however, two main differences between MTHF and FA. The first one is that MTHF does not always bind to the active site of FRa. Unlike FA, it can spend extended periods away from the pocket (see below the detailed discussion of the interactions in Conf1). The second major dissimilarity is that even when MTHF binds to the active center of the receptor, the attraction is by far not as strong as that of FA. There are several evidences for that - the lower intensities of the RDF peaks (Figure S34), preference for interaction of $A B$ only with one of the target partners (Trp102 in Conf4) or with none at all (Figure S35), smaller number and/or shorter existence times of the hydrogen bonds of glutamate (Figure S36 and Table S6). The most striking example is that of Conf3 where MTHF has practically no specific interactions with the target amino acids. It tries to compensate with extensive hydrogen bonding with Arg106 (Figure S36) or with pronounced $\pi$-stacking with Arg61 in Conf4 (Figure S38) but this is not enough to keep the molecule bound permanently to the active site of the receptor. The binding in Conf4 is also weaker because the glutamate of MTHF is practically not involved in interactions with the pocket (Figure S34). The enhanced mobility of the ligand at the surface of FR $\alpha$ is confirmed also by the analysis of the closest atom pairs from the ligand and the protein in the second bound period of Conf2 and in the two bound segments of Conf3 (Figure S37). The data show that within these periods MTHF scans significant portion of the protein, only partially interacting with pocket residues (Conf2 and Conf3, 0-130 ns) or not coupling to them at all (Conf3, 160-200 ns).

None of these (even though partial) specific interactions is present in Conf1. There, the glutamate of MTHF does not form any hydrogen bonds with the target pocket residues, although this part of the ligand points toward the active site. The RDFs maxima of glutamate and THP are at distances $>2 \mathrm{~nm}$, signifying lack of interaction with the pocket. Instead, MTHF communicates intensively both with the GPI anchor and with the lipid bilayer (Figure S39). While the coupling to the membrane is more dynamic, MTHF is steadily attracted to the GPI (ca. 1000 contacts) throughout the entire bound period. The data in Figure S40 show that the ligand is quite immobilized slightly above the membrane interface and interacts predominantly with one sphingomyelin and several phosphatidylcholines. 


\section{Configuration 2}
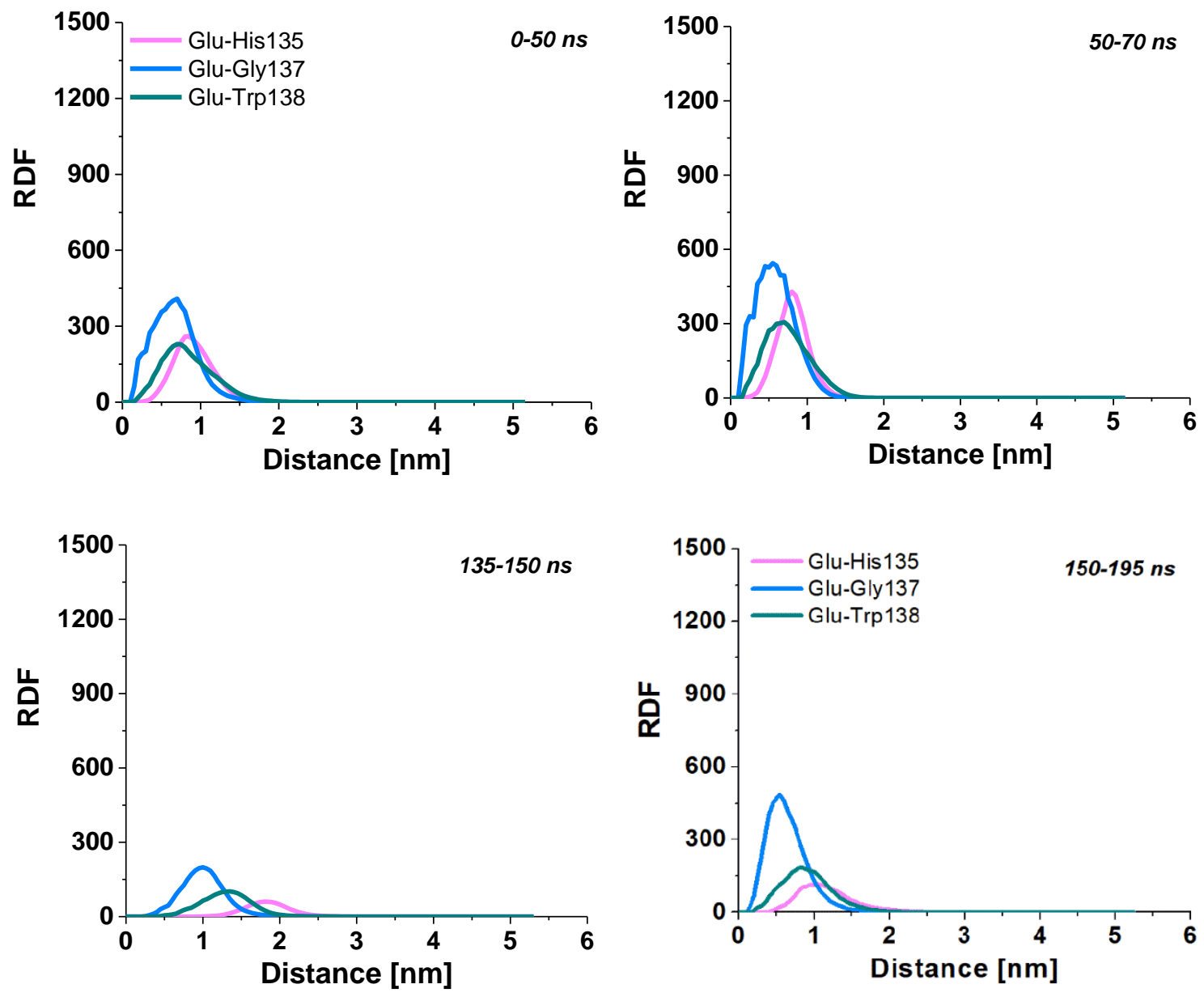

\section{Configuration 3}
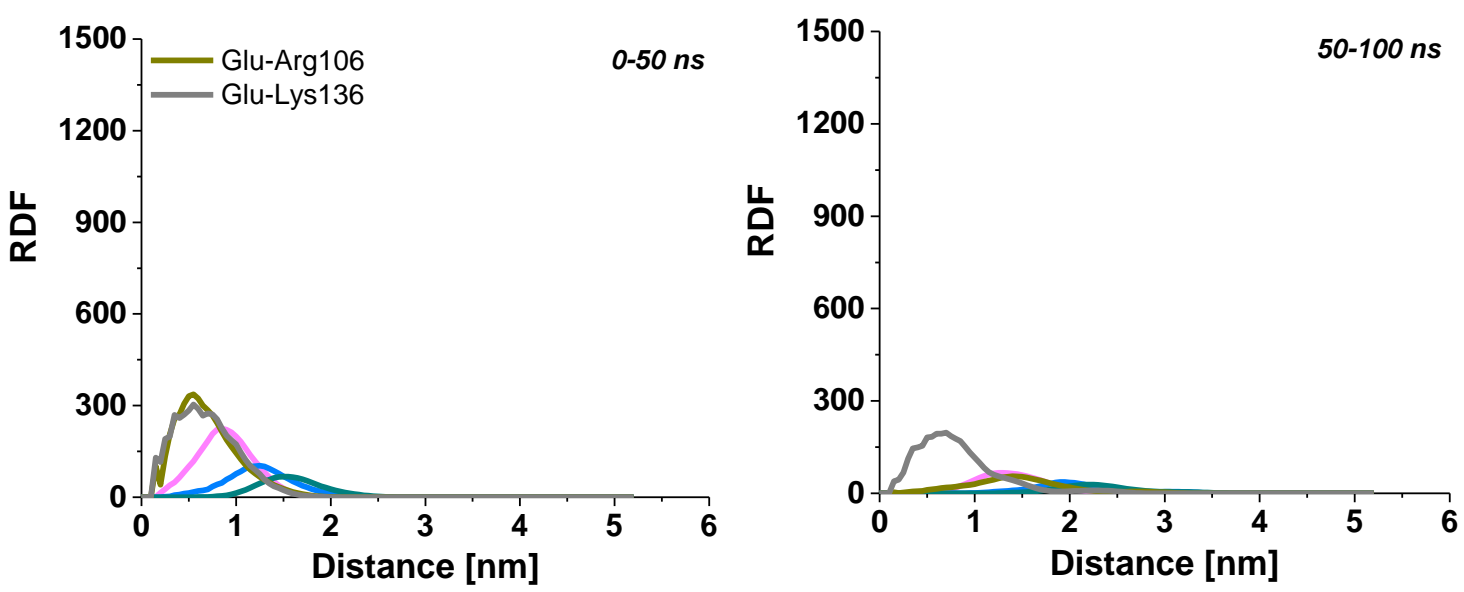

Figure S34: Radial distribution functions of the distance between the glutamate part of MTHF and its target interaction partners from FR $\alpha$ during the bound periods of Conf 2 to Conf 4 
Configuration 3

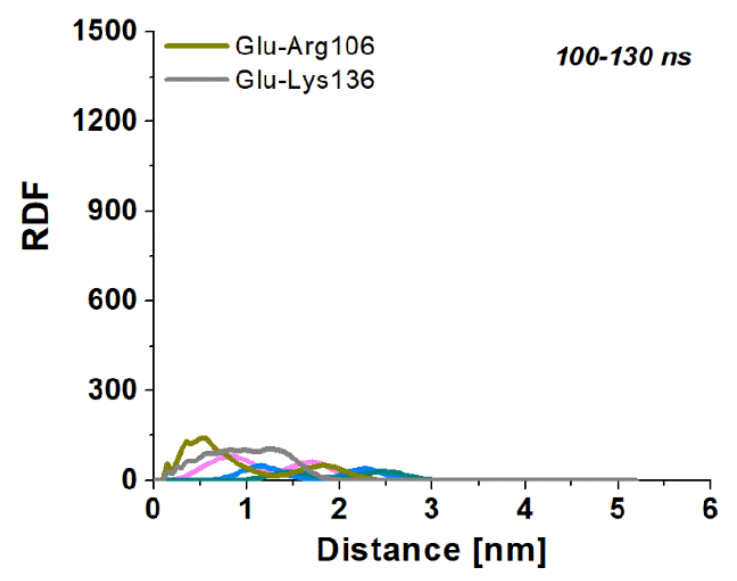

\section{Configuration 4}
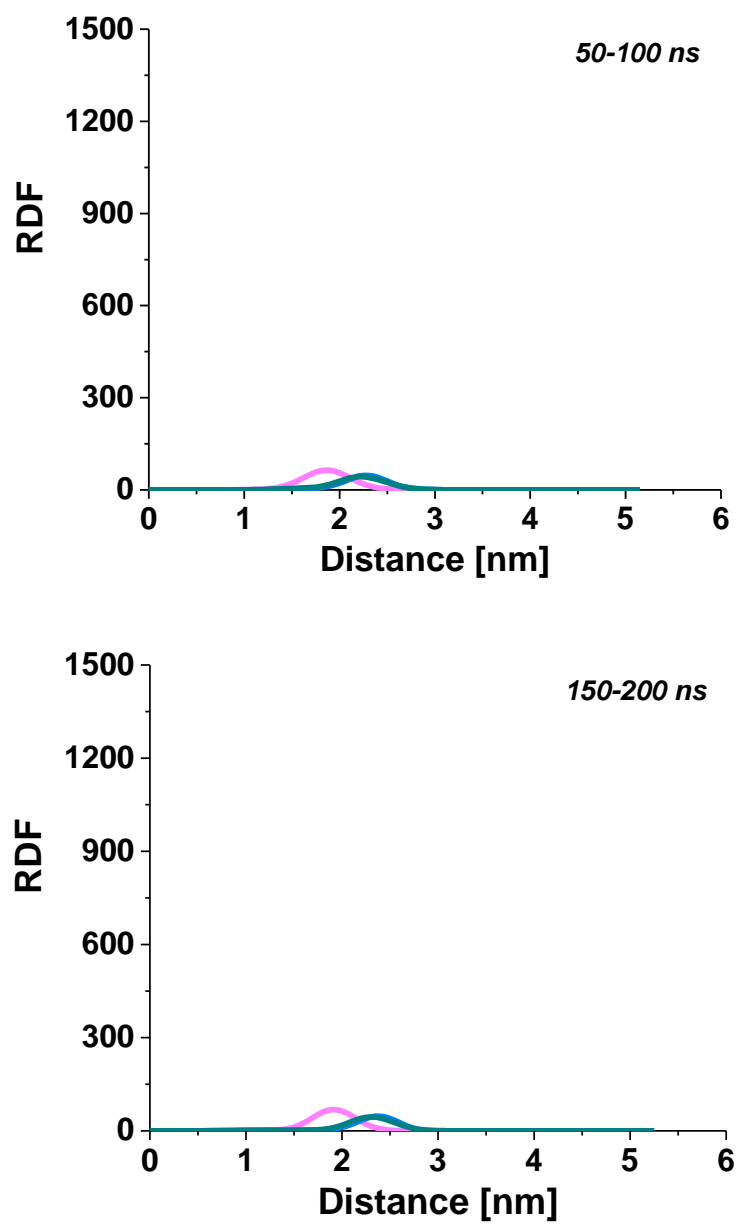

Figure S34 (Continued)
Configuration 4
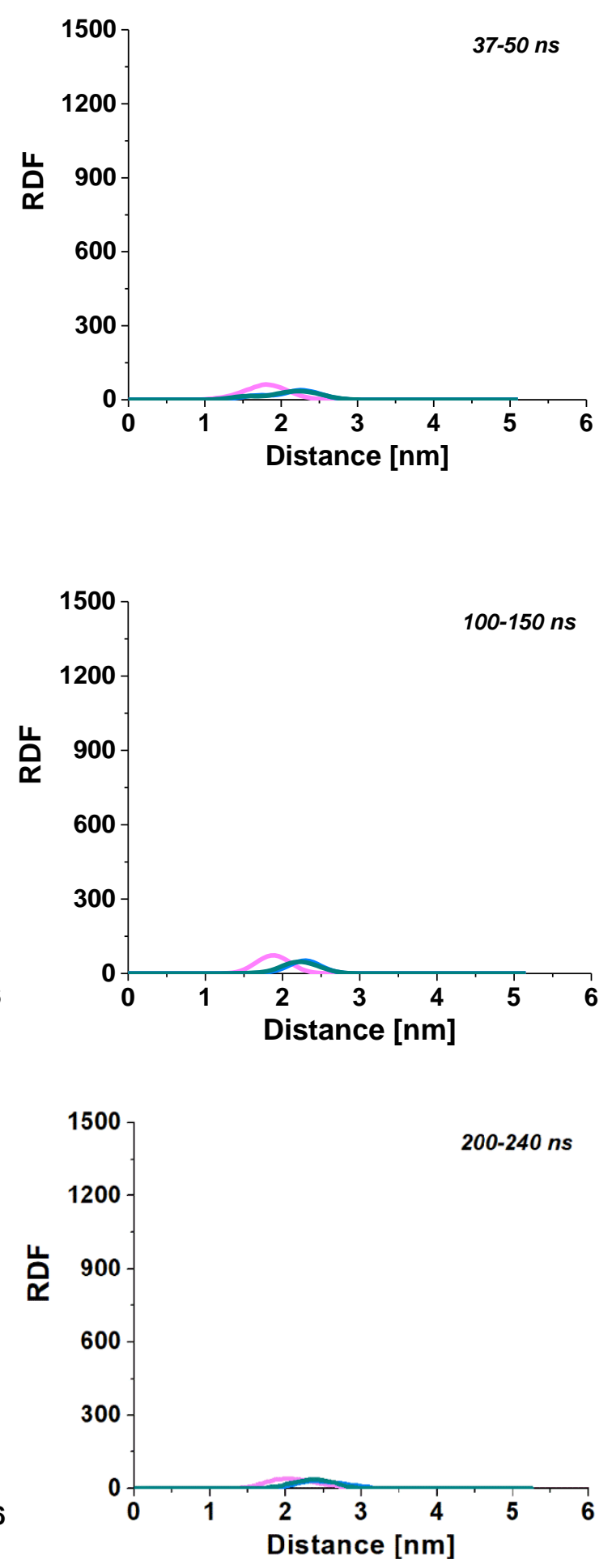


\section{Configuration 2}
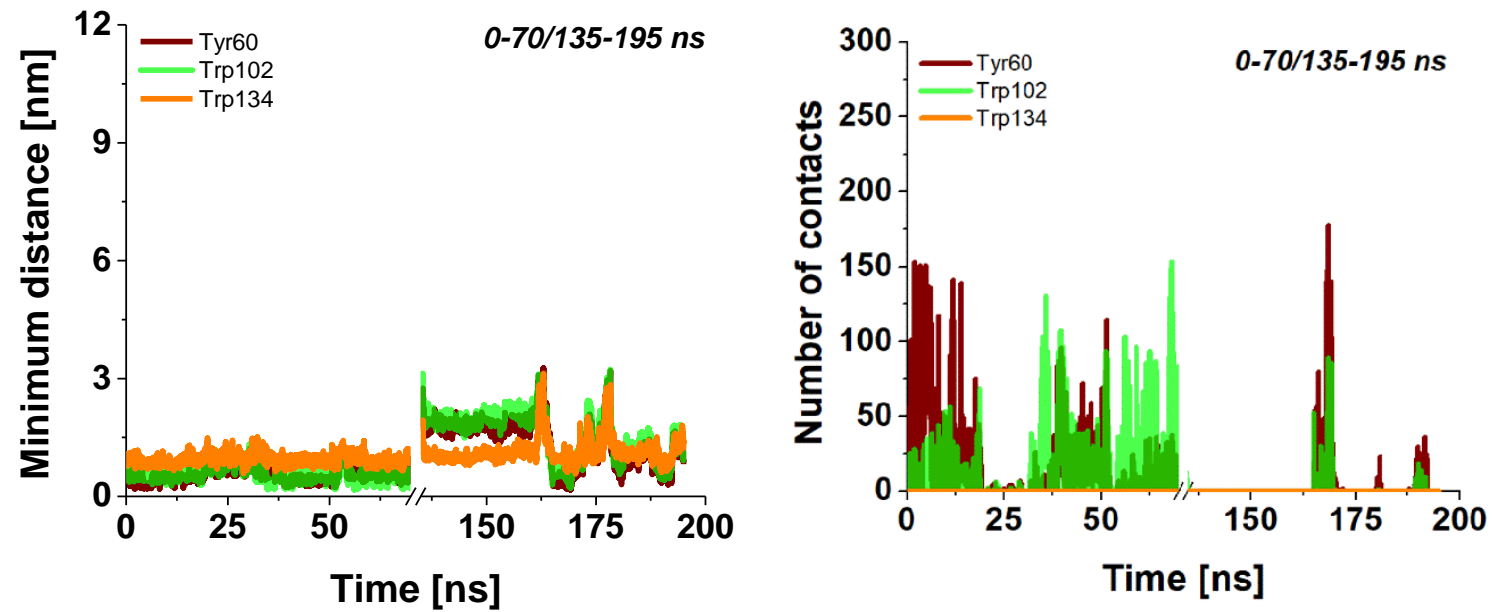

Configuration 3
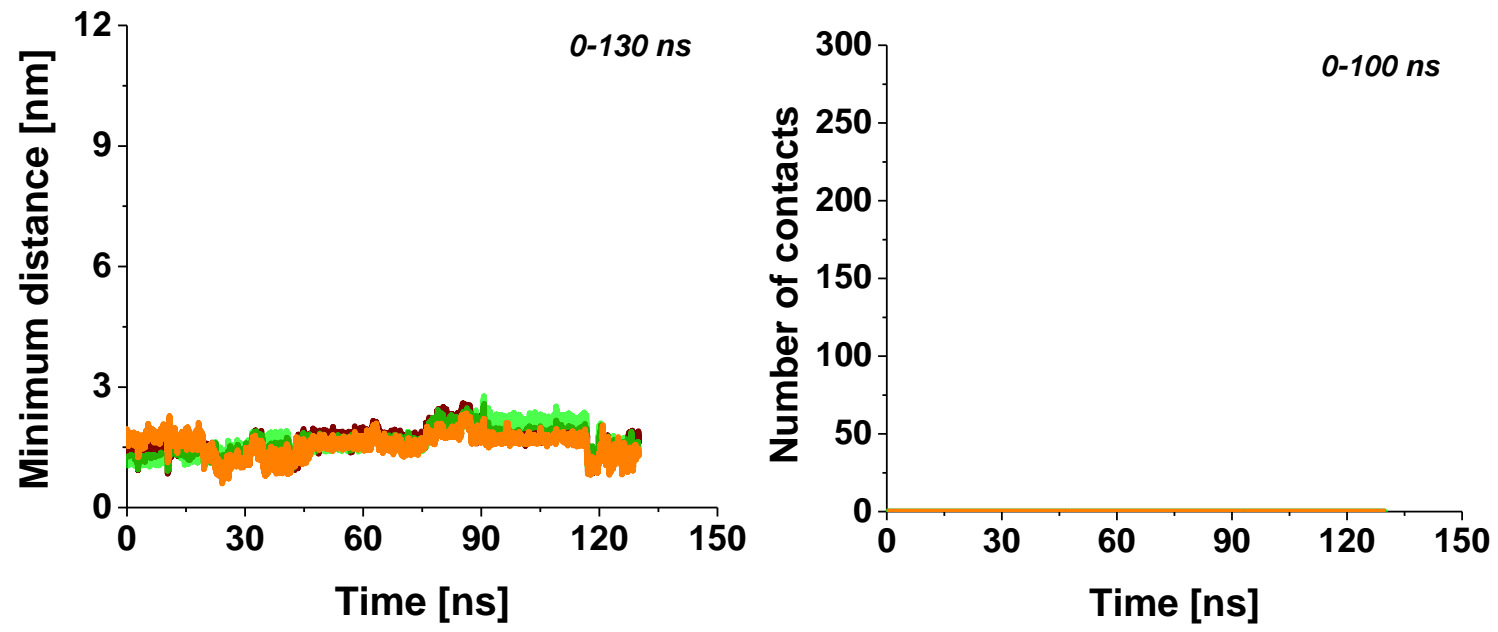

\section{Configuration 4}
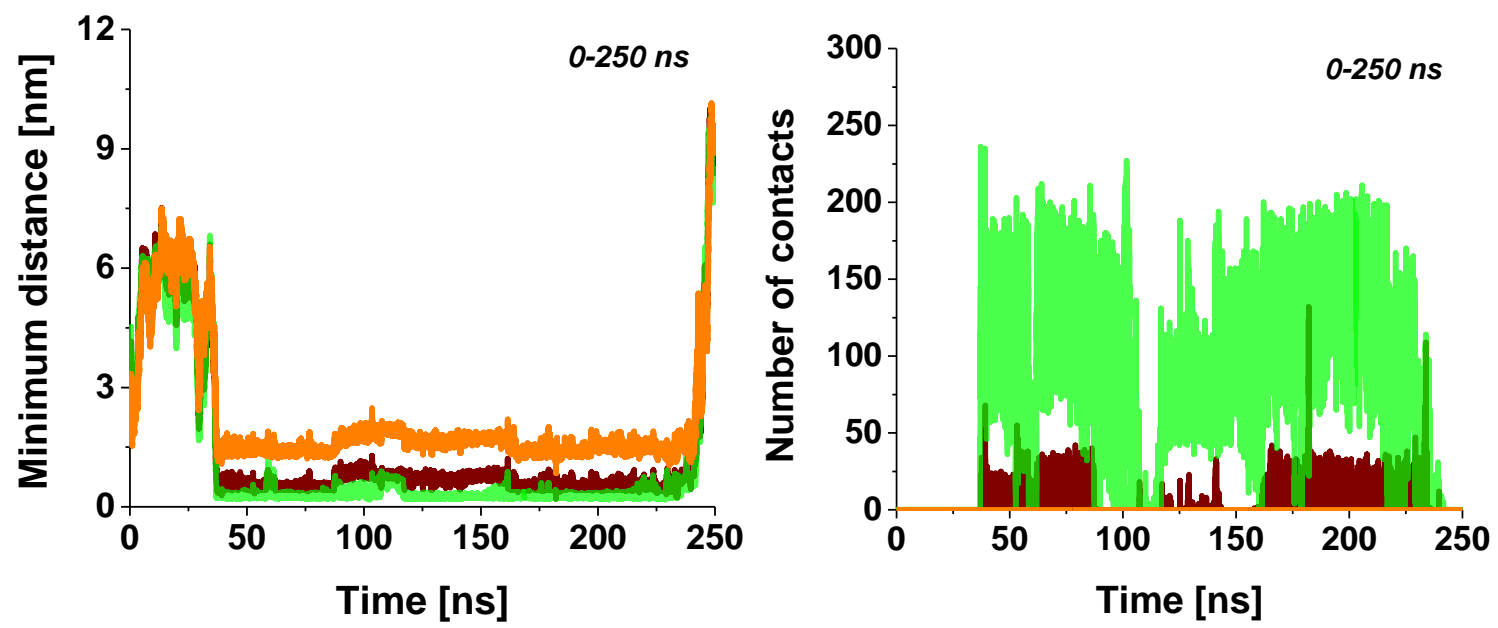

Figure S35: Evolution of the (left) minimum distance and (right) number of contacts during the entire bound period between atom pairs from the $A B$ fragment of MTHF and its target interaction partners from FR $\alpha$ in Conf2 to Conf4 
Table S6: Average number $\left(\mathrm{N}_{\mathrm{HB}}{ }^{\text {ave }}\right)$ with standard deviation, preferred length $\left(\mathrm{R}_{\mathrm{HB}}{ }^{\text {ave }}\right)$, and type of the hydrogen bonds formed between the glutamate of MTHF and its target interaction partners from FR $\alpha$ during the bound periods of Conf2 and Conf3

\begin{tabular}{|c|c|c|c|c|}
\hline Amino acid & Period & $\mathrm{N}_{\mathrm{HB}}$ ave & $R_{H B}{ }^{\text {ave }}, n m$ & Bond type \\
\hline \multicolumn{5}{|c|}{ Configuration 2} \\
\hline \multirow{2}{*}{ Trp102 } & $0-70 \mathrm{~ns}$ & $0.001 \pm 0.034$ & $0.218 / 0.188$ & \multirow{2}{*}{$\alpha$-carboxylate group } \\
\hline & $135-195 \mathrm{~ns}$ & $0.000 \pm 0.000$ & ----- & \\
\hline \multirow{2}{*}{ Lys136 } & $0-70 \mathrm{~ns}$ & $0.299 \pm 0.501$ & 0.168 & \multirow{2}{*}{ both carboxylate groups } \\
\hline & $135-195 \mathrm{~ns}$ & $0.101 \pm 0.317$ & 0.168 & \\
\hline \multirow[b]{2}{*}{ Trp140 } & $0-70 \mathrm{~ns}$ & $0.201 \pm 0.404$ & 0.183 & \multirow{2}{*}{ both carboxylate groups } \\
\hline & $135-195 \mathrm{~ns}$ & $0.045 \pm 0.210$ & 0.208 & \\
\hline \multicolumn{5}{|c|}{ Configuration $3^{\#}$} \\
\hline \multirow[b]{3}{*}{ Lys136 } & $0-50 \mathrm{~ns}$ & $0.413 \pm 0.522$ & 0.168 & \multirow{3}{*}{$\begin{array}{l}\text { both carboxylate groups; only } \\
\gamma \text {-carboxylate group in the } \\
\text { middle time period }\end{array}$} \\
\hline & $50-100 \mathrm{~ns}$ & $0.014 \pm 0.116$ & 0.163 & \\
\hline & $100-130 \mathrm{~ns}$ & $0.219 \pm 0.460$ & 0.163 & \\
\hline \multirow[b]{3}{*}{ Arg106 } & $0-50 \mathrm{~ns}$ & $0.951 \pm 0.923$ & 0.173 & \multirow{3}{*}{$\begin{array}{l}\Theta=\mathrm{N} \oplus \mathrm{i}- \\
\text { both carboxylate groups; only } \\
\gamma \text {-carboxylate group in the } \\
\text { middle time period }\end{array}$} \\
\hline & $50-100 \mathrm{~ns}$ & $0.343 \pm 0.594$ & $0.173 / 0.178$ & \\
\hline & $100-130 \mathrm{~ns}$ & $0.210 \pm 0.588$ & 0.173 & \\
\hline
\end{tabular}

\#No H-bonds are formed with Trp102 and Trp140 in Conf3 


\section{Configuration 2}
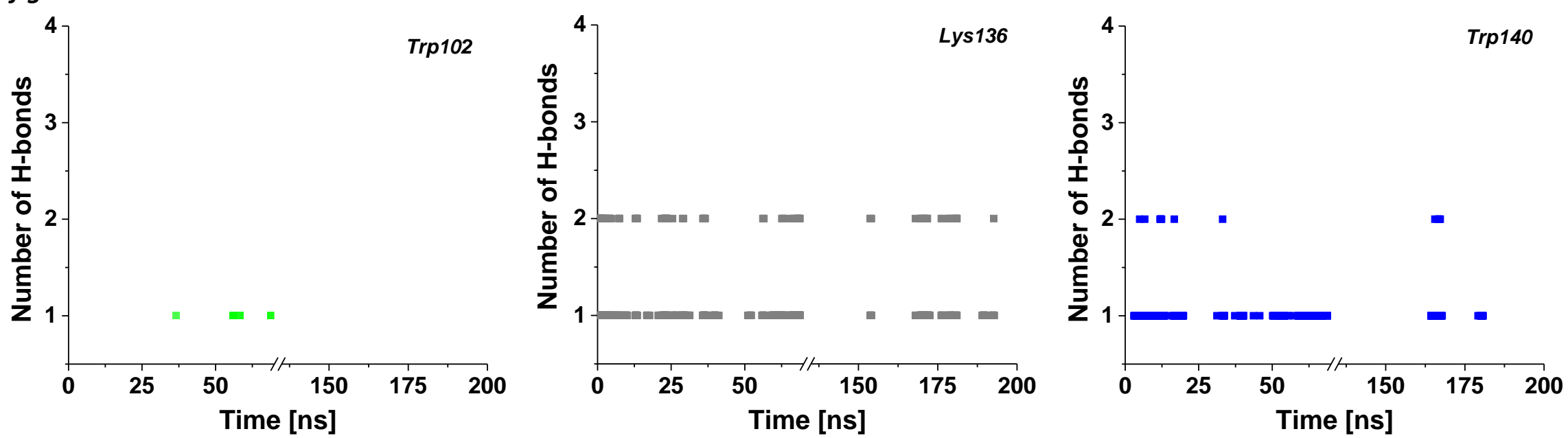

\section{Configuration 3}
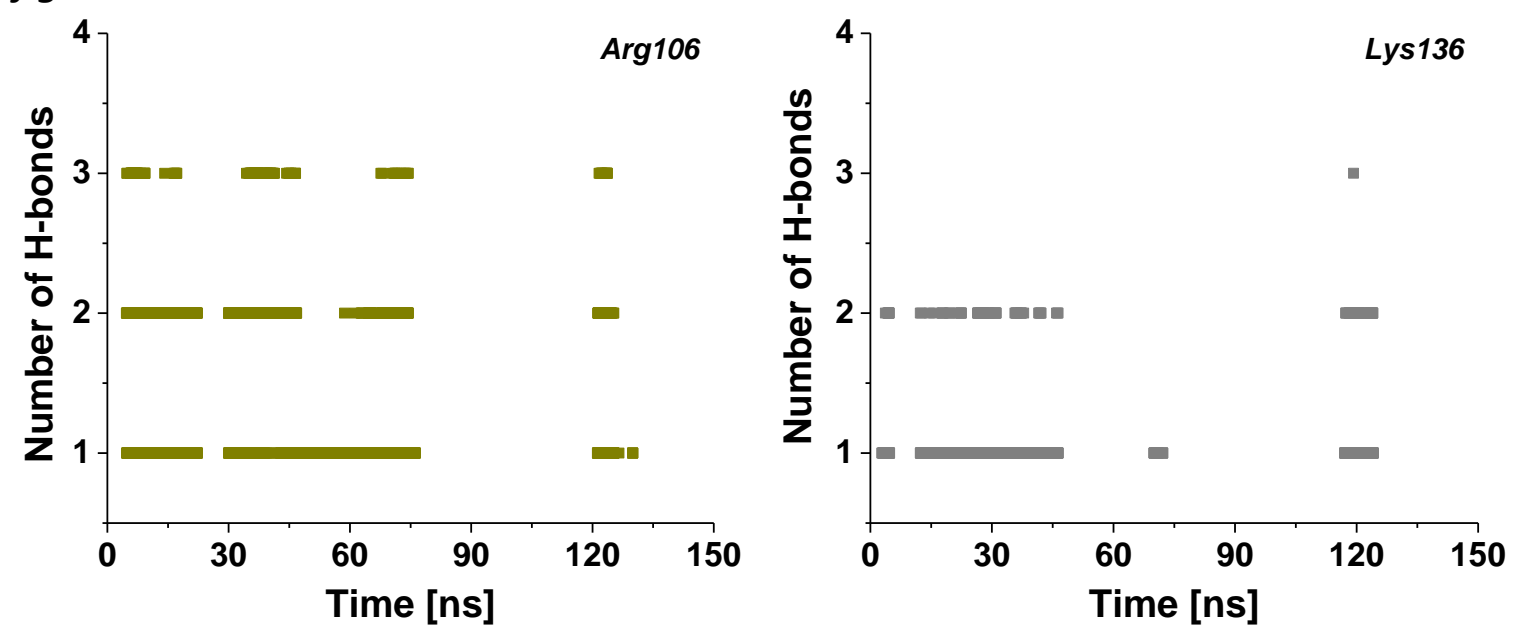

Figure S36: Evolution of the number of hydrogen bonds between the glutamate of MTHF and its target interaction partners from the binding pocket of FR $\alpha$ during the bound periods of Conf2 and Conf3; no hydrogen bonds are formed between these entities in Conf4 and no $\mathrm{H}$-bonds are registered with Trp102 and Trp140 in Conf3 

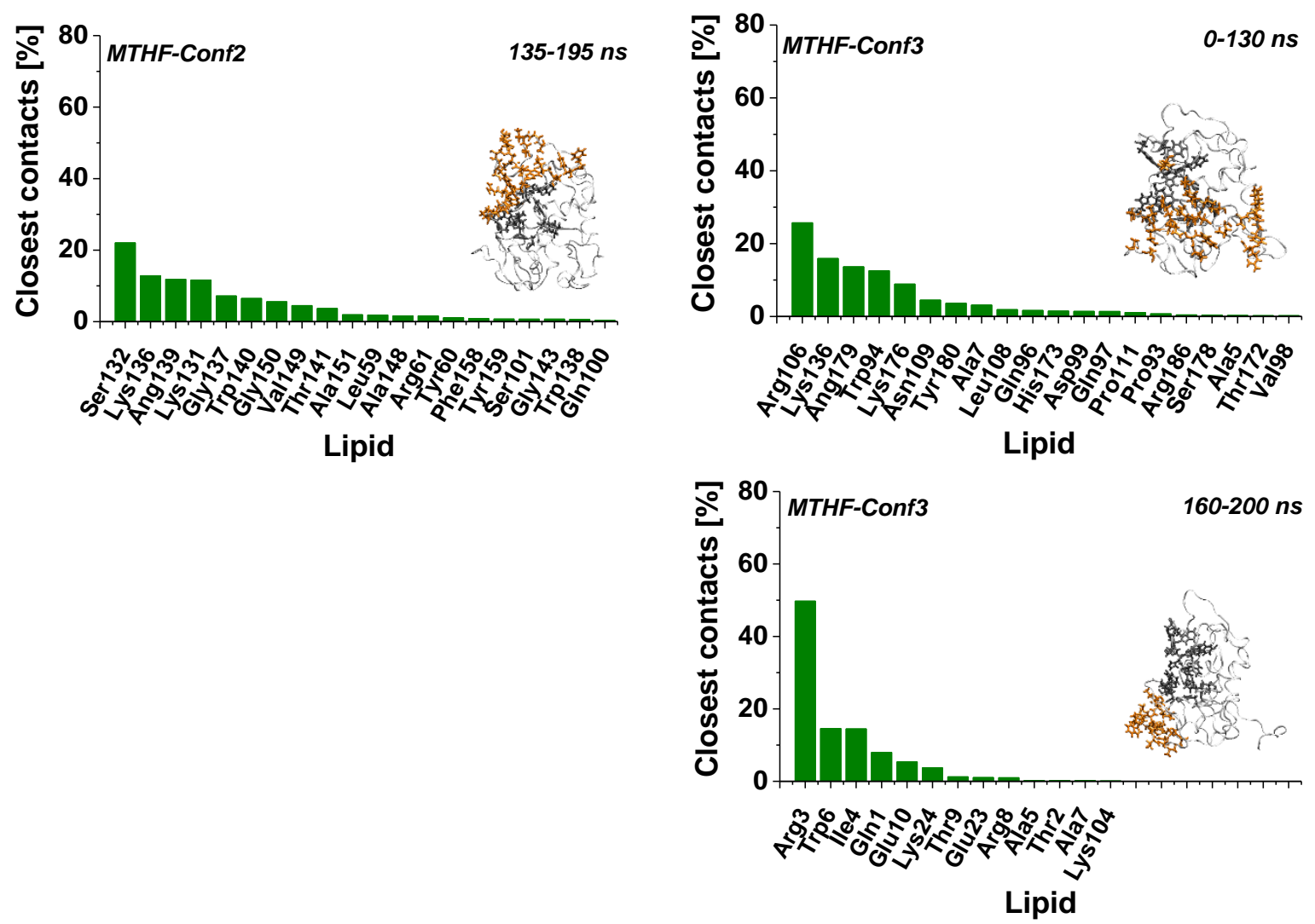

Figure S37: Distribution of the number of minimum distances closed between MTHF and amino acids from FR $\alpha$ during the bound periods of (left) Conf2 and (right) Conf3; illustration of these interaction partners on the structure of the receptor from the last frame of the particular period is given as inset, where the interaction partners of MTHF are depicted in orange licorice 


\section{Configuration 4}
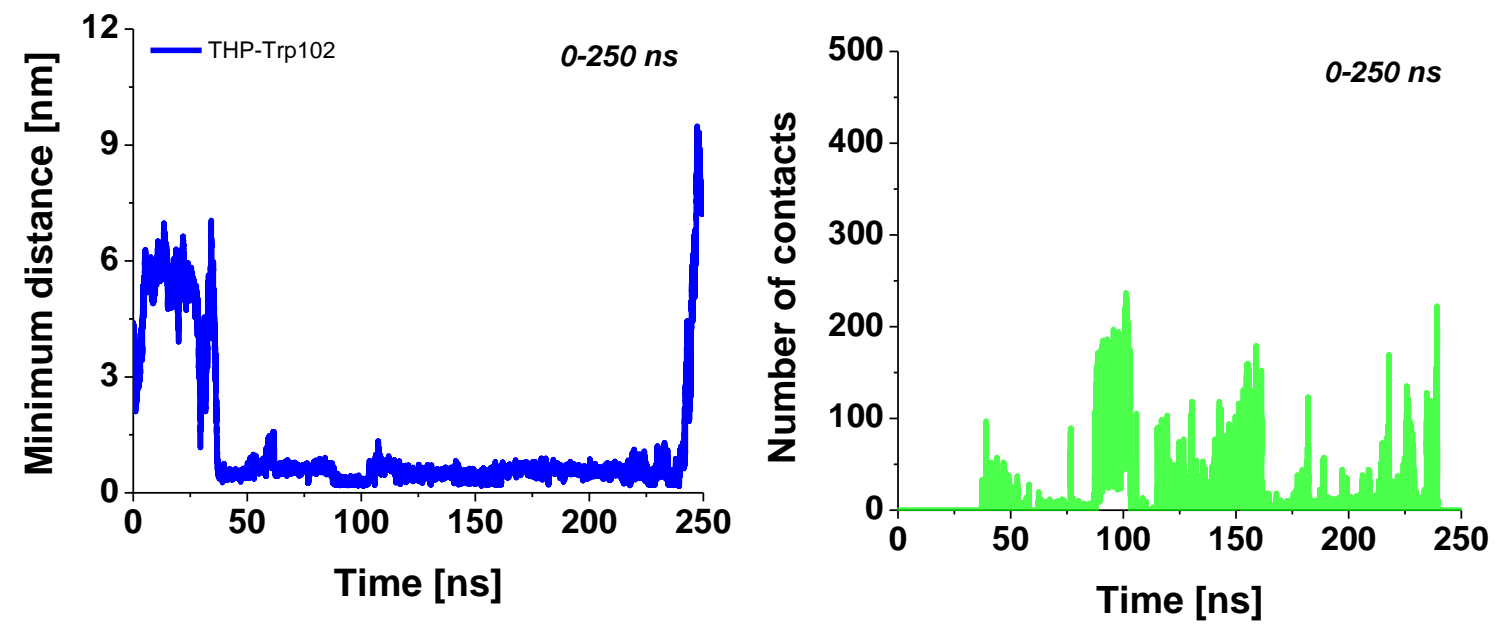

(A)
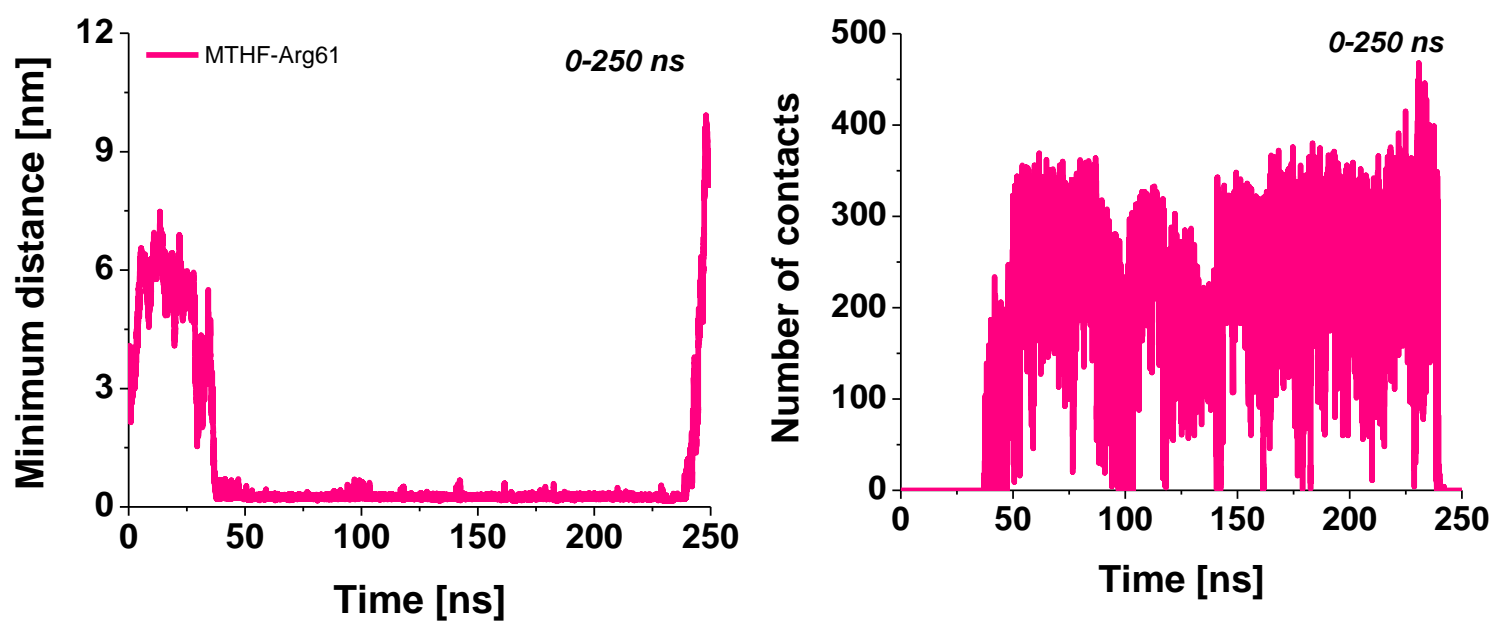

Figure S38: Evolution of the (left) minimum distance and (right) number of contacts between atom pairs from the (A) THP fragment of MTHF and Trp102 from FR $\alpha$ and (B) the whole ligand and Arg61 from FR $\alpha$ during the entire bound period of Conf4 


\section{Configuration 1}
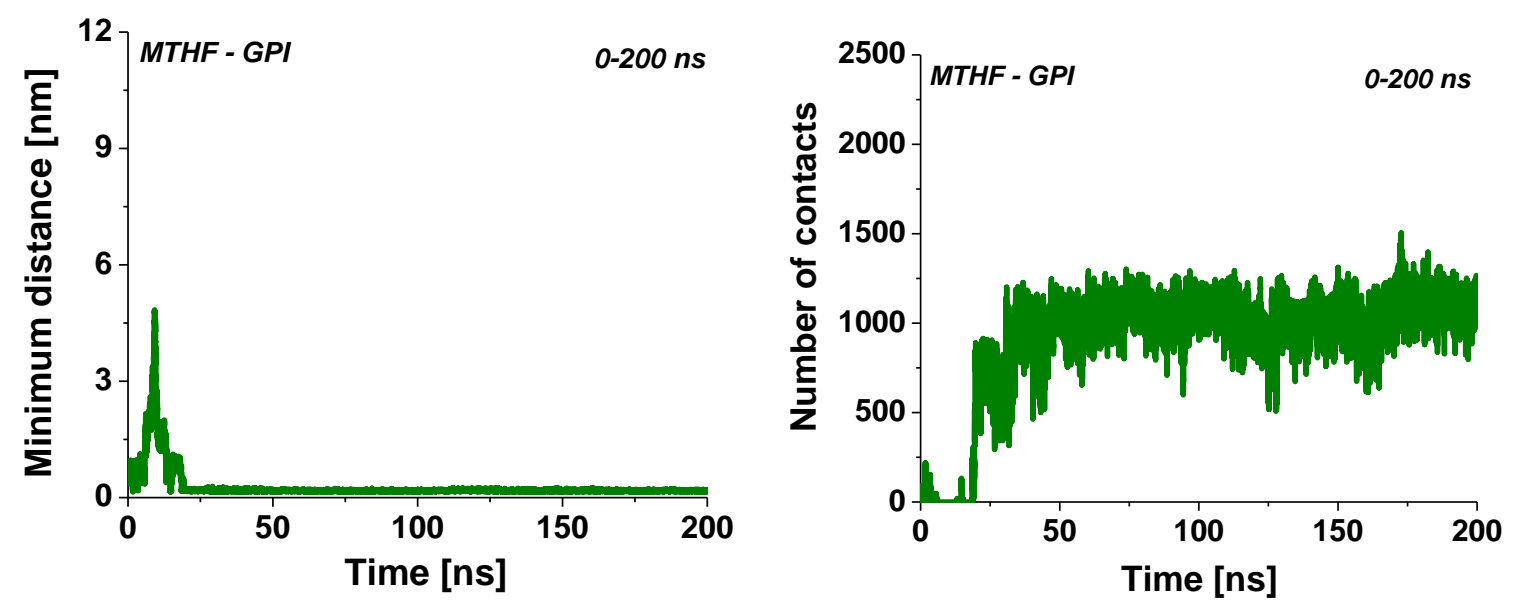

(A)
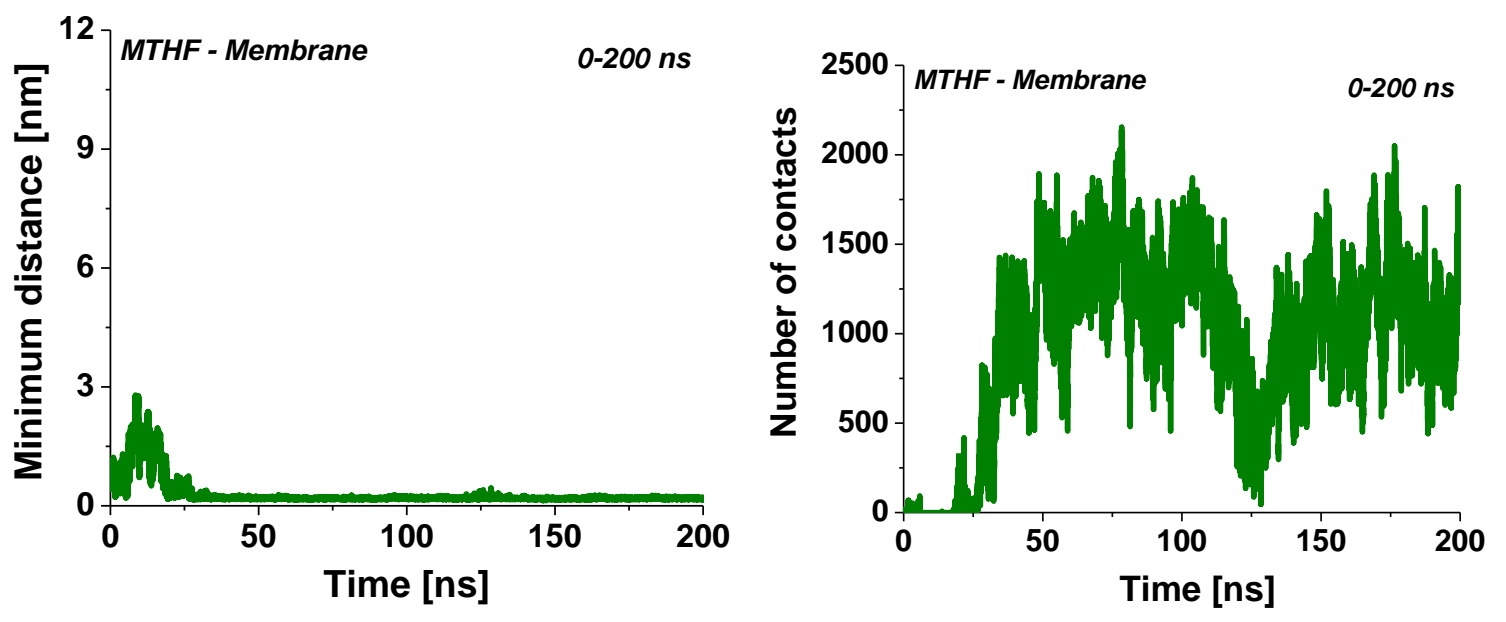

(B)

Figure S39: Evolution of the (left) minimum distance and (right) number of contacts between atom pairs from MTHF and (A) the GPI anchor or (B) membrane lipids during the entire bound period of Conf1 

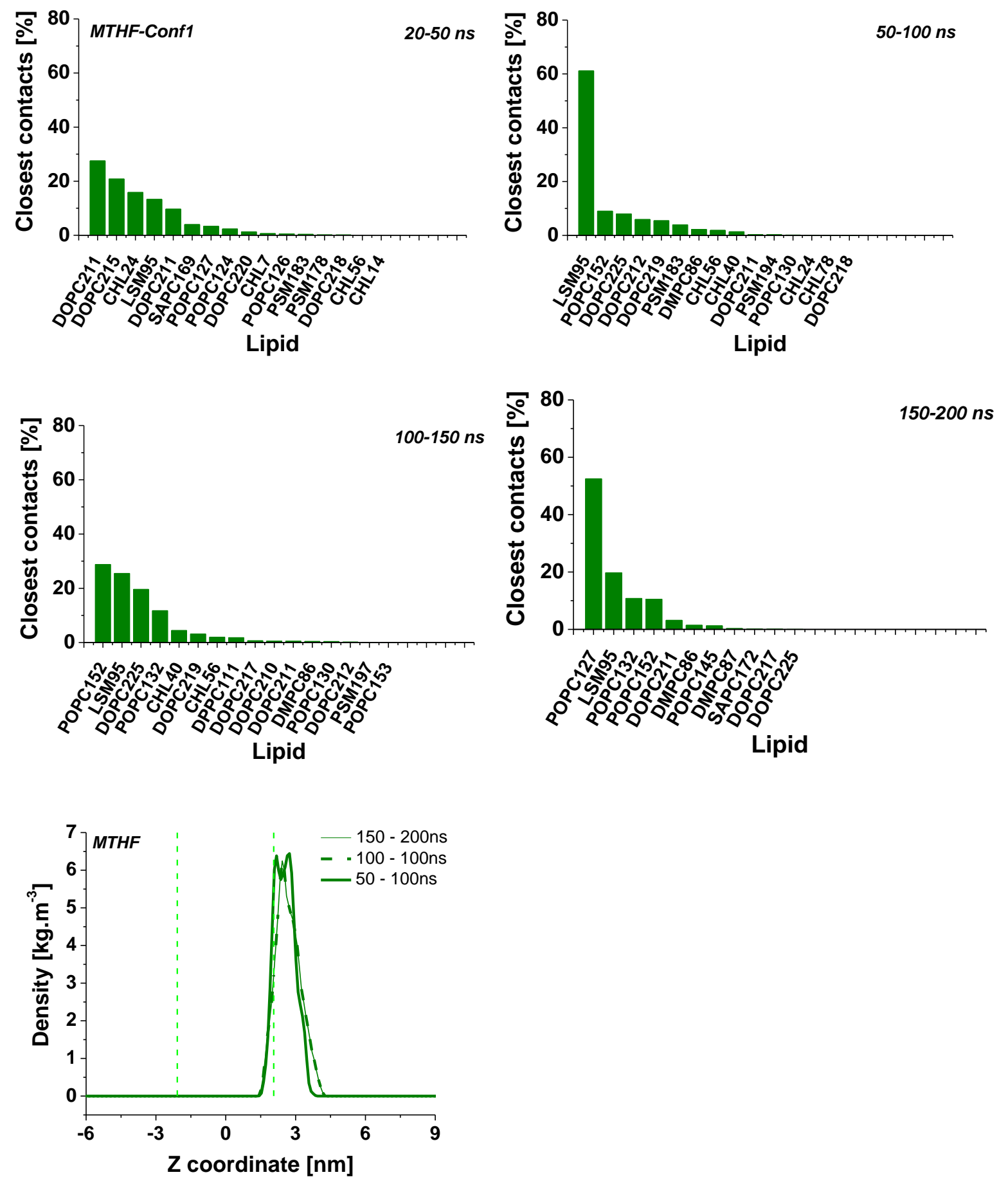

Figure S40: (top) Distribution of the closest distances between pairs of atoms from MTHF and from membrane lipids and (bottom) mass density profiles of MTHF in direction normal to the interface during the bound period of Conf1; the dashed lines denote the equimolecular dividing surfaces (drawn at water density of $500 \mathrm{~kg} \cdot \mathrm{m}^{-3}$ ) and $\mathrm{z}=0$ is the middle of the lipid bilayer 


\section{Pteroyl ornithine}

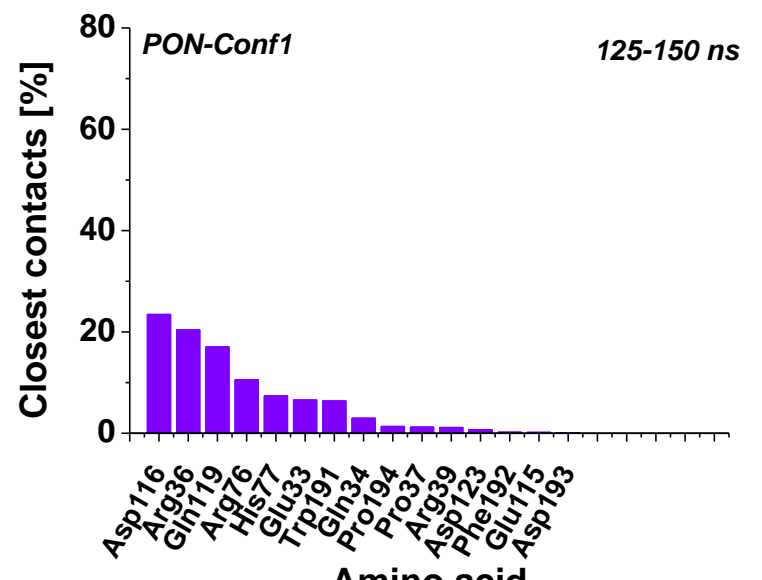

Amino acid
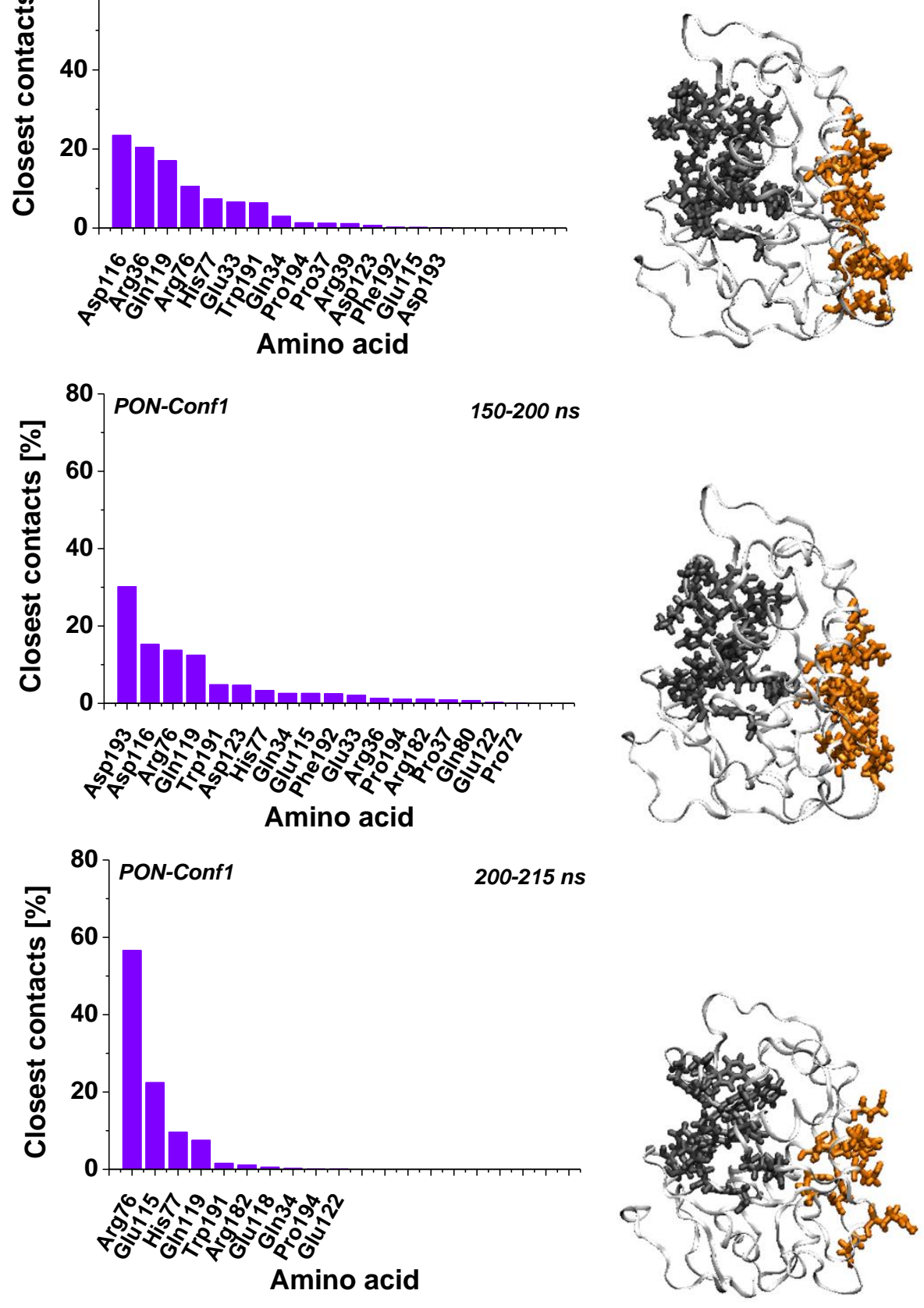

Figure S41: Distribution of the number of minimum distances closed between PON and amino acids from FR $\alpha$ during the bound period of Conf1; illustration of these interaction partners on the structure of the receptor on the last frame of the particular period is given next to the histograms, where the interaction partners of PON are depicted in orange licorice 


\section{Effect of the binding on the ligands and receptor structure}
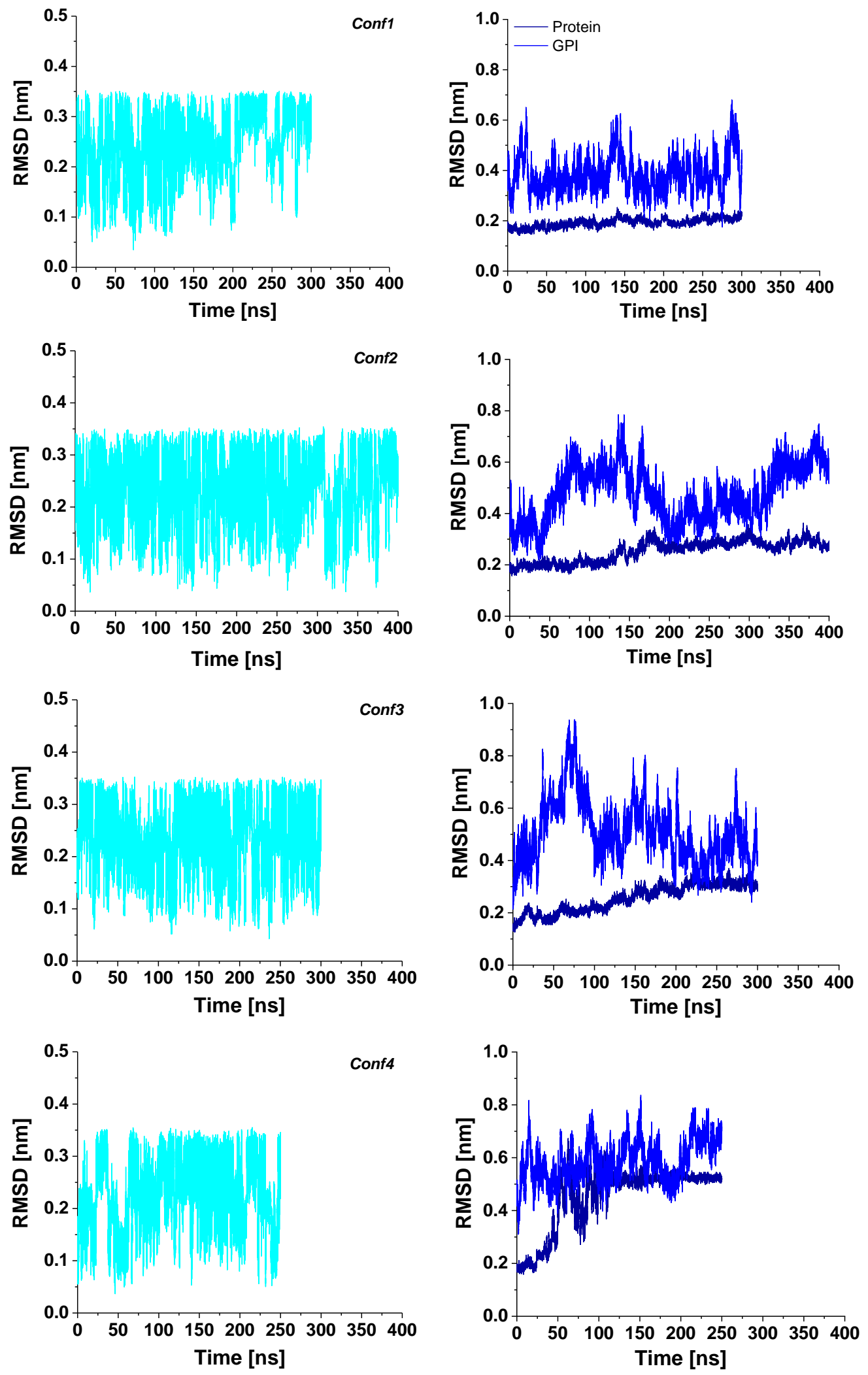

Figure S42: Evolution of the RMSD of the atomic coordinates of (left) folate and (right) FRa and the GPI anchor in the four MD trajectories; the energy-minimized initial structure is used as a reference 

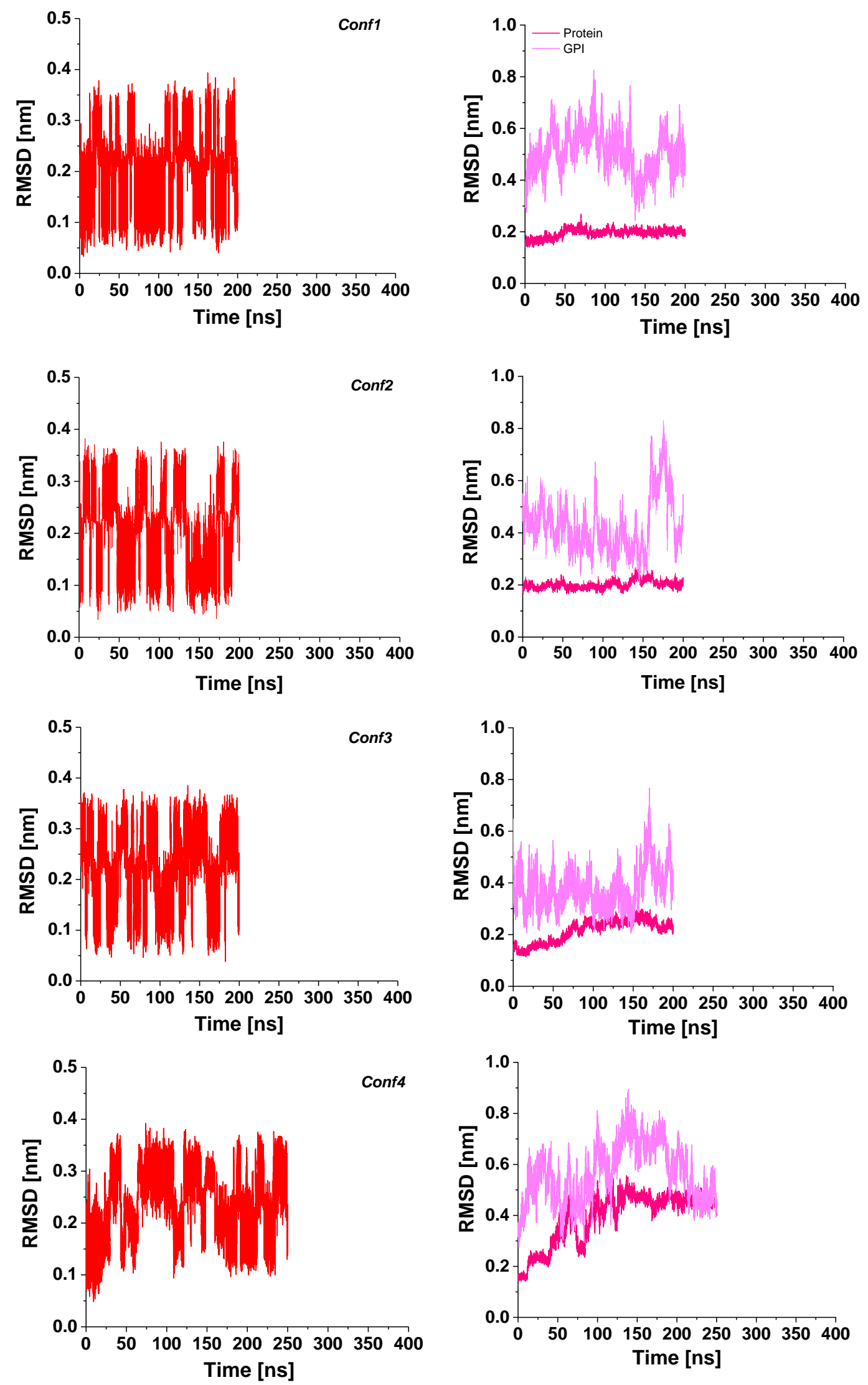

Figure S43: Evolution of the RMSD of the atomic coordinates of (left) methotrexate and (right) FR $\alpha$ and the GPI anchor in the four MD trajectories; the energy-minimized initial structure is used as a reference 

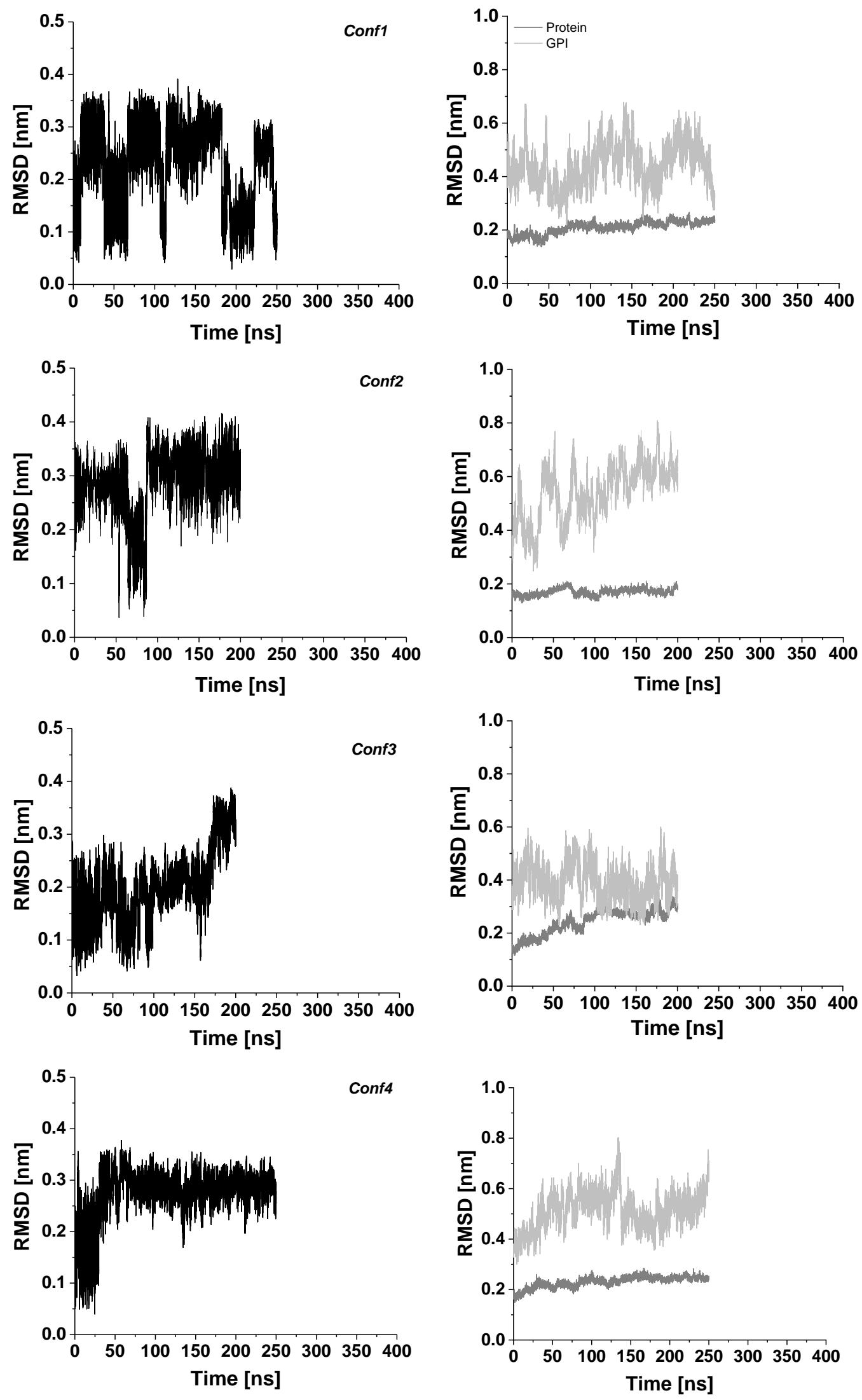

Figure S44: Evolution of the RMSD of the atomic coordinates of (left) raltitrexed and (right) FR $\alpha$ and the GPI anchor in the four MD trajectories; the energy-minimized initial structure is used as a reference 

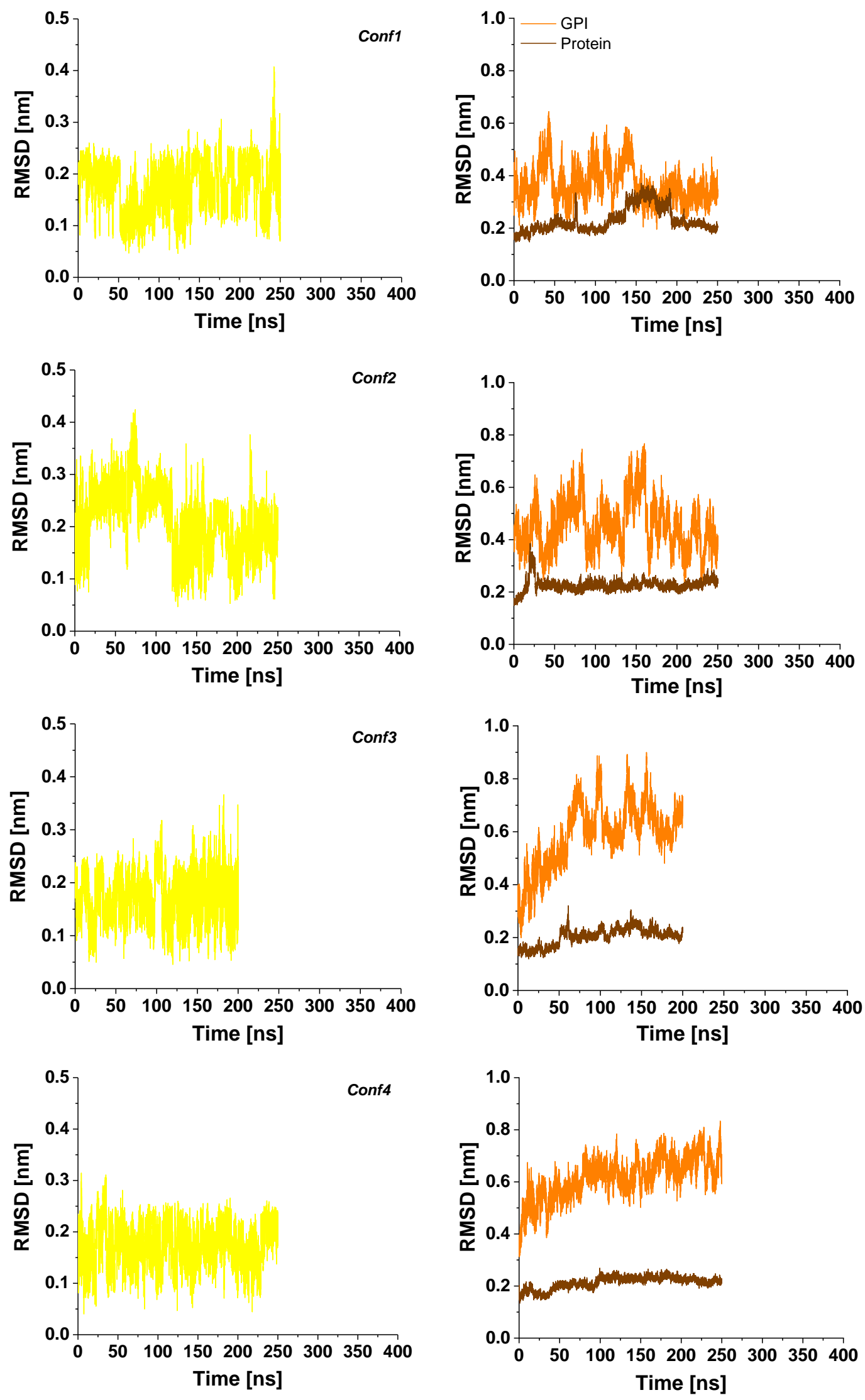

Figure S45: Evolution of the RMSD of the atomic coordinates of (left) pemetrexed and (right) FR $\alpha$ and the GPI anchor in the four MD trajectories; the energy-minimized initial structure is used as a reference 

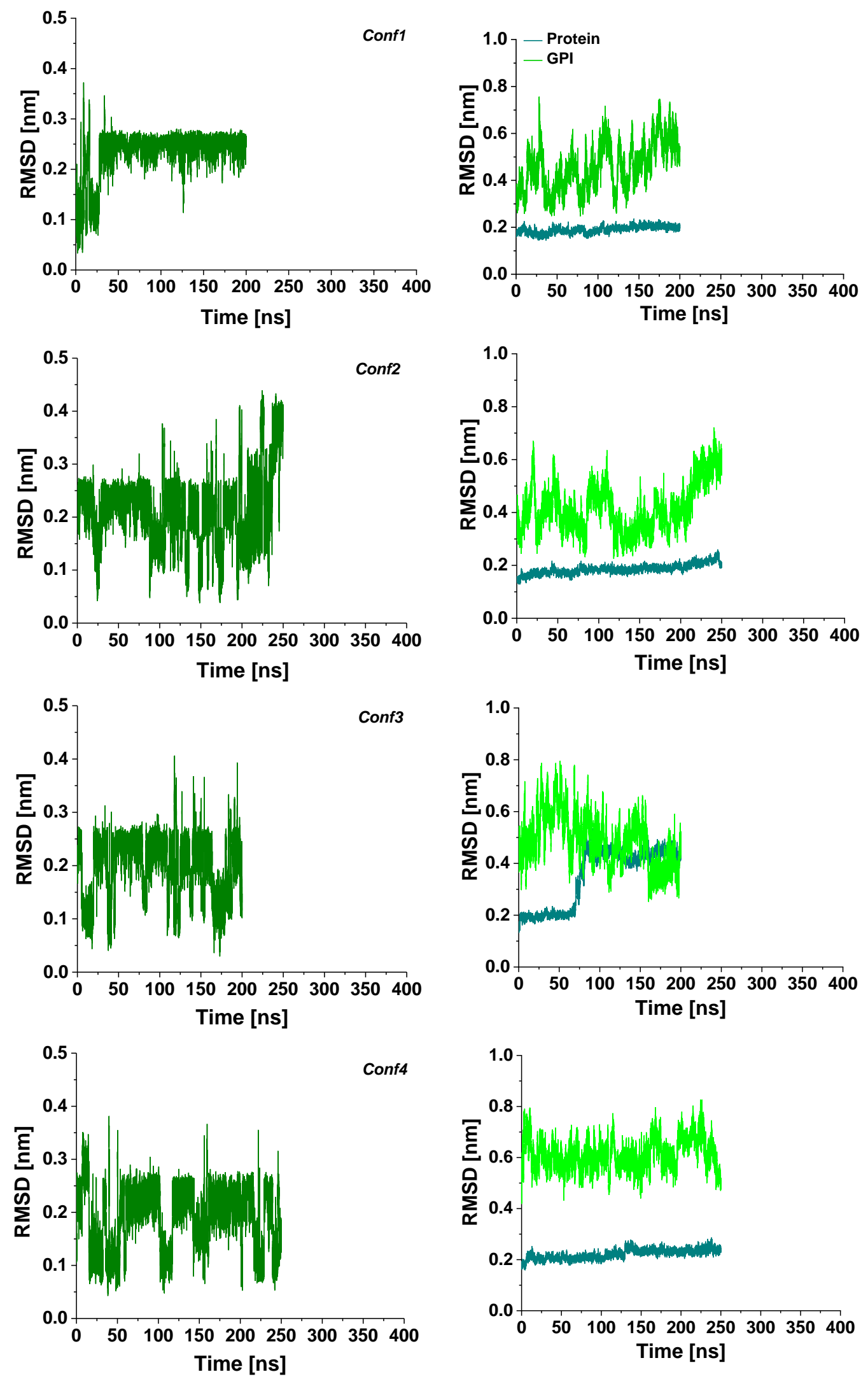

Figure S46: Evolution of the RMSD of the atomic coordinates of (left) MTHF and (right) FR $\alpha$ and the GPI anchor in the four MD trajectories; the energy-minimized initial structure is used as a reference 

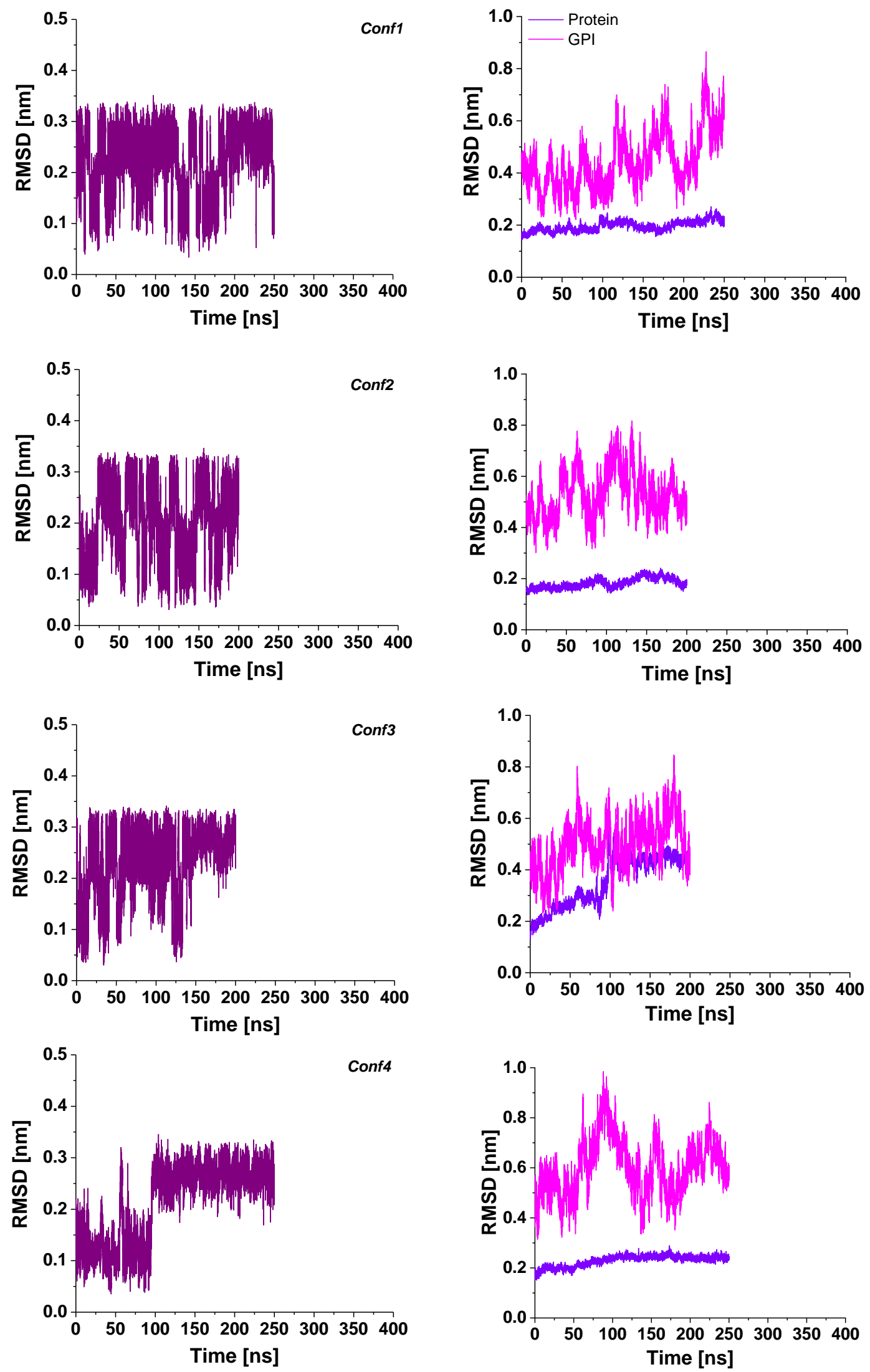

Figure S47: Evolution of the RMSD of the atomic coordinates of (left) pteroyl ornithine and (right) FR $\alpha$ and the GPI anchor in the four MD trajectories; the energy-minimized initial structure is used as a reference 
The RMSD profiles show that the ligands either have no relaxation periods at the beginning of the trajectories or they are very short ( $<20 \mathrm{~ns})$. After that the fluctuations of the vectors structure have equilibrium profiles. When the ligand is not bound to the protein, the dynamics of the conformational changes evolves in a very similar way to that of the unbound molecule. ${ }^{\mathrm{S} 15}$ The GPI anchor is very mobile and has large and fast fluctuations with no common pattern in the different trajectories. This coincides with the behavior of this fragment in the unperturbed membrane/receptor system, where the anchor was very mobile in normal direction to the interface. ${ }^{516}$ The receptor has a relatively long relaxation but in all cases reaches a steady RMSD profile lasting at least $100 \mathrm{~ns}$.

The structure of the ligands reacts differently when they bind to FR $\alpha$. RTX in Conf3 is the most perturbed one - its RMSD increases gradually when the molecule is inserted deep into the pocket. This verifies the assumption that the ligand needs to undergo a significant geometry change in order to enter tightly into the binding site. The structure of RTX is also quite immobilized in Conf4, where it is tightly locked in upright position between two loops of FR $\alpha$. In the other two trajectories the structure of the ligand fluctuates even after binding. The RMSD of FA also reacts to the complexation with the receptor. In all four trajectories, when the ligand binds there is a period between 10 ns and 50 ns when the RMSD gradually decreases. After that the fluctuations of the folate geometry are restored. It may be assumed that FA needs this initial period for adjustment at the pocket entrance and after that it continues fluctuating, waiting for an opportunity to enter the pocket interior. The geometry of the other four ligands is practically unaffected by the binding. This is especially interesting for PTX because it is adsorbed for much extended periods of time on the protein surface. Nevertheless, this does not influence significantly its conformation. Maybe this is part of the reasons for the 'stickiness' of this ligand.

There is no visible change of the protein RMSD upon binding or unbinding of any of the ligands. This means that the complex formation does not induce any global change of the protein structure. After 158 ns of Conf3 of RTX, when the ligand enters deeply into the pocket, there is some fluctuation of the RMSD of FR $\alpha$. However, it is of the same order of magnitude as the one observed earlier in the trajectory and, hence, cannot be ascribed definitively to the binding process. It should be mentioned that FR $\alpha$ undergoes major structural alterations (RMSD up to 0.5 $\mathrm{nm}$ ) in Conf4 of FA, Conf3 of MTHF, and Conf4 of MTX. This may be the reason why all three ligands dissociate in these trajectories ca. 20 ns after the changes of the protein structure start.

The GPI anchor also does not react in any consistent way to the binding of the ligands. A notable exception is MTHF in Conf1, where the vector molecule interacts directly with the GPI sugars (Figure S12). There, the RMSD of the anchor gradually increases over the trajectory, reacting to the tight coupling of the ligand. The RMSD of the GPI also shows a drift in Conf2 of RTX, where the ligand is bound at the base of the protein and is located between it and the membrane, also relatively close to the anchor. 

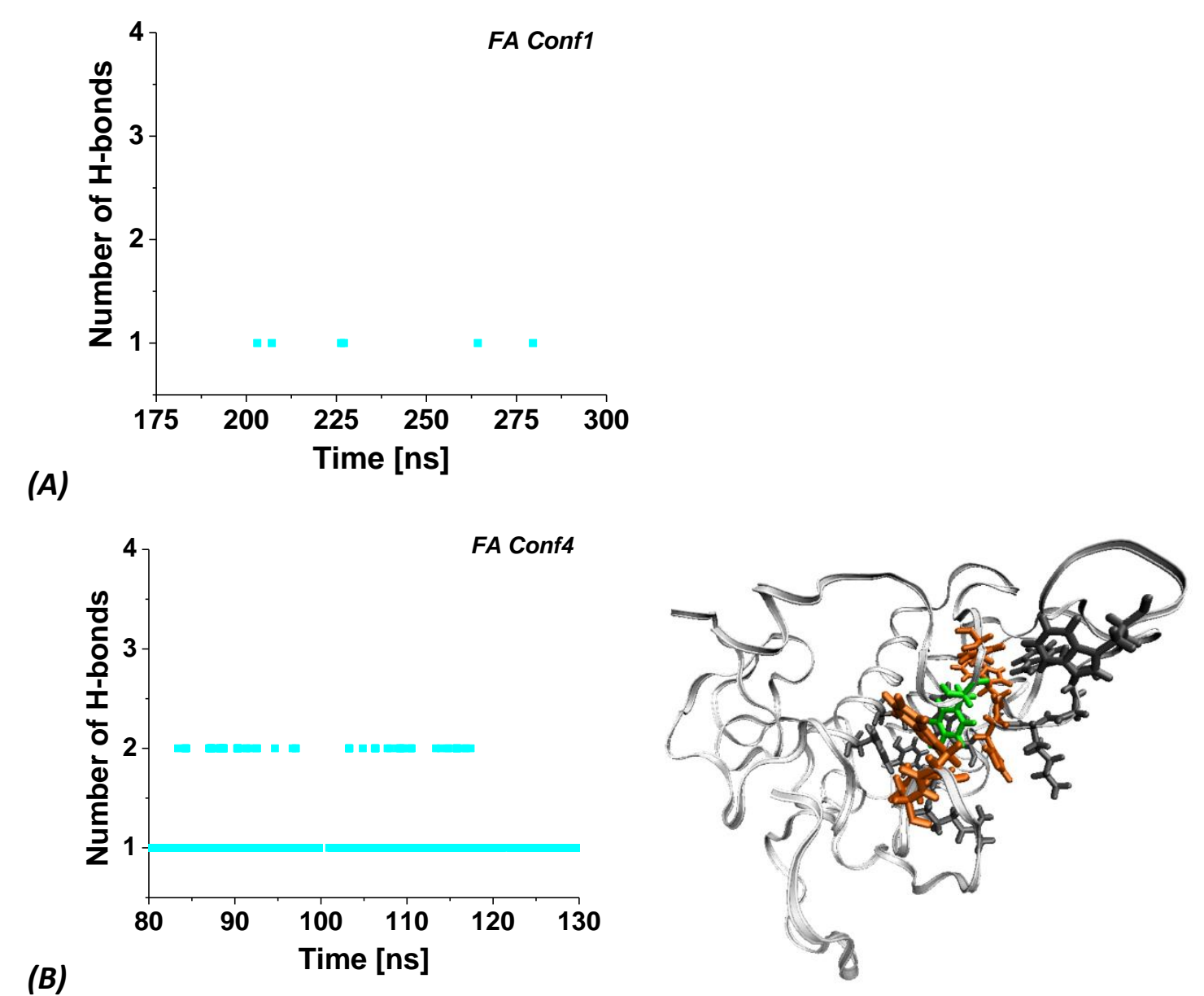

(B)
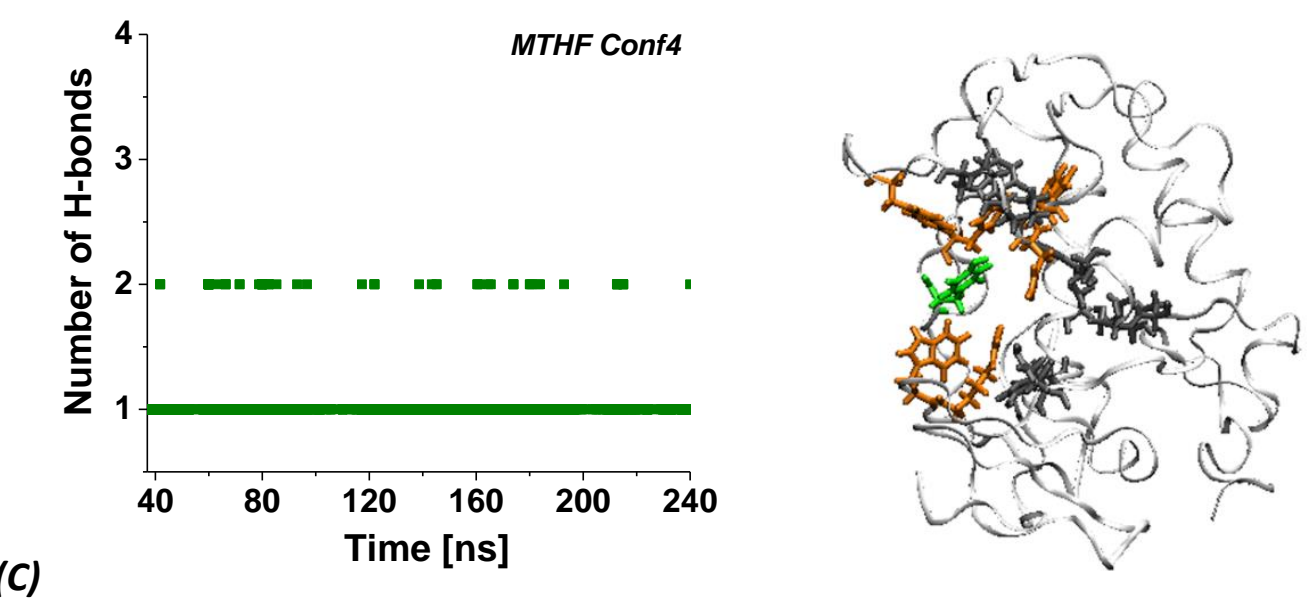

Figure S48: (left) Evolution of the number of hydrogen bonds between Tyr60 and the rest of the pocket residues in (A) FA Conf1 during the entire bound period, (B) FA Conf4 during the second bound section, and (C) MTHF Conf4 during the entire bound period; (right) visual representation of the protein (white ribbons) with pocket residues in grey licorice, Tyr60 in green, and other residues involved in hydrogen bonding with Tyr60 in orange 


\section{References}

S1) Gocheva, G.; Ivanova, N.; lliev, S.; Petrova, J.; Madjarova, G.; Ivanova, A. Characteristics of a folate receptor- $\alpha$ anchored into a multilipid bilayer obtained from atomistic molecular dynamics simulations. J. Chem. Theory Comput. 2020, 16, 749-764.

S2) Yu, Y.; Skočaj, M.; Kreft, M. E.; Resnik, N.; Veranič, P.; Franceschi, P.; Sepčic, K.; Guella, G. Comparative lipidomic study of urothelial cancer models: association with urothelial cancer cell invasiveness. Mol. Biosyst. 2016, 12, 3266-3279.

S3) Chen, C.; Ke, J.; Zhou, X. E.; Yi, W.; Brunzelle, J. S.; Li, J.; Yong, E.; Xu, H. E.; Melcher, K. Structural basis for molecular recognition of folic acid by folate receptors. Nature 2013, 500, 486-489.

S4) Sharma, P.; Varma, R.; Sarasij, R. C.; Ira; Gousset, K.; Krishnamoorthy, G.; Rao, M.; Mayor, S. Nanoscale organization of multiple GPI-anchored proteins in living cell membranes. Cell 2004, 116, 577-589.

S5) a) Darden, T.; York, D.; Pedersen, L. Particle mesh Ewald: An $N \cdot \log (\mathrm{N})$ method for Ewald sums in large systems. J. Chem. Phys. 1993, 98, 10089-10092; (b) Essmann, U.; Perera, L.; Berkowitz, M. L.; Darden, T.; Lee, H.; Pedersen, L. G. A smooth particle mesh Ewald method. J. Chem. Phys. 1995, 103, 8577-8593; (c) Toukmaji, A.; Sagui, C.; Board, J.; Darden, T.; Efficient particle-mesh Ewald based approach to fixed and induced dipolar interactions. J. Chem. Phys. 2000, 113, 10913-10927.

S6) Bussi, G.; Donadio, D.; Parrinello, M. Canonical sampling through velocity rescaling. J. Chem. Phys. 2007, 126, 014101.

S7) Berendsen, H. J. C.; Postma, J. P. M.; van Gunsteren, W. F.; DiNola, A.; Haak, J. R. Molecular dynamics with coupling to an external bath. J. Chem. Phys. 1984, 81, 3684-3691.

S8) Ryckaert, J.-P.; Ciccotti, G.; Berendsen, H. J. C. Numerical integration of the Cartesian equations of motion of a system with constraints: molecular dynamics of n-alkanes. J. Comput. Phys. 1977, 23, 327- 341.

S9) Miyamoto, S.; Kollman, P. A. A. Settle: An analytical version of the SHAKE and RATTLE algorithm for rigid water models. J. Comput. Chem. 1992, 13, 952-962.

S10) Wibowo, A. S.; Singh, M.; Reeder. K. M.; Carter, J. J.; Kovach, A. R.; Meng, W.; Ratnam, M.; Zhang, F.; Dann III, C. E. Structures of human folate receptors reveal biological trafficking states and diversity in folate and antifolate recognition. Proc. Natl. Acad. Sci. USA 2013, 110, 15180-15188.

S11) Mauritz, R.; Peters, G. J.; Kathmann, I.; Teshale, H.; Noordhuis, P.; Comijn, E. M.; Pinedo, H. M.; Jansen, G. Dynamics of antifolate transport via the reduced folate carrier and the membrane folate receptor in murine leukaemia cells in vitro and in vivo. Cancer Chemother. Pharmacol. 2008, 62, 937-948

S12) Theti, D. S.; Jackman, A. L. The Role of Folate Receptor-Mediated Transport in the Antitumor Activity of Antifolate Drugs, Clin. Cancer Res. 2004, 10, 1080-1089.

S13) Wang, X.; Shen, F.; Freisheim, J. H.; Gentry, L. E.; Ratnam, M. Differential stereospecificities and affinities of folate receptor isoforms for folate compounds and antifolates. Biochem. Pharmacol. 1992, 44, 1898-1901.

S14) Petrova, J.; Gocheva, G.; Ivanova, N.; Iliev, S.; Atanasova, B.; Madjarova, G.; Ivanova, A. Molecular simulation of the structure of folate and antifolates at physiological conditions. J. Molec. Graph. Model. 2019, 87, 172-184.

S15) Iliev, S.; Gocheva, G.; Ivanova, N.; Atanasova, B.; Petrova, J.; Madjarova, G.; Ivanova A. Identification and computational characterization of isomers with cis and trans amide bonds in folate and its analogues. Phys. Chem. Chem. Phys. 2018, 20, 28818-28831.

S16) Gocheva, G.; Ivanova, N.; Iliev, S.; Petrova, J.; Madjarova, G.; Ivanova, A. Characteristics of a folate receptor- $\alpha$ anchored into a multilipid bilayer obtained from atomistic molecular dynamics simulations. J. Chem. Theory Comput. 2020, 16, 749-764. 